\title{
Carbonyl-stabilized Phosphorus Ylide as an Organocatalyst for Cyanosilylation Reactions Using TMSCN
}

\footnotetext{
Wen-Biao Wu, ${ }^{\dagger}$ Xing-Ping Zeng ${ }^{\dagger \neq, *}$ and Jian Zhou ${ }^{\dagger \S, *}$

${ }^{\dagger}$ Shanghai Engineering Research Center of Molecular Therapeutics and New Drug Development, and ${ }^{\dagger}$ Shanghai Key Laboratory of Green Chemistry and Chemical Process, East China Normal University, Shanghai 200062, P. R. China; E-mail for J. Zhou: jzhou@chem.ecnu.edu.cn

$\ddagger$ Key Laboratory of Small Functional Organic Molecule, Ministry of Education, Jiangxi Normal University, Nanchang, Jiangxi, 330022, P. R. China; E-mail for X.-P. Zeng: 005173@jxnu.edu.cn

${ }^{\S}$ State Key Laboratory of Organometallic Chemistry, Shanghai Institute of Organic Chemistry, Shanghai 200032, P. R. China
}

\begin{tabular}{|l|c|}
\hline Contents & Page \\
\hline 1. Mechanistic studies & S2-S5 \\
\hline 1-1. NMR studies & S2-S3 \\
\hline 1-2. In situ IR studies & S4-S5 \\
\hline 1-2-1. IR spectrum of 1a and 1e & S4 \\
\hline 1-2-2. IR study the activation of 1d to TMSCN & S5 \\
\hline 2. NMR spectra & S6-S82 \\
\hline
\end{tabular}




\section{Mechanistic studies.}

\section{1-1. NMR studies}

The ${ }^{13} \mathrm{C}$ NMR (126 MHz) and ${ }^{31} \mathrm{P}$ NMR (202 MHz) studies were recorded at room temperature in $\mathrm{CD}_{3} \mathrm{CN}$. The general procedure was as follows: to a $5 \mathrm{~mm}$ NMR tube containing $0.5 \mathrm{~mL} \mathrm{CD} \mathrm{CN}_{3}$ was sequentially added TMSCN and then 1e was added in three portions. After the solid was fully dissolved, the mixture was balanced at room temperature for 30 min before detection. The ${ }^{31} \mathrm{P}$ NMR study was conducted using $\mathrm{H}_{3} \mathrm{PO}_{4}(\delta=0.00 \mathrm{ppm})$ as the external standard.

As shown in Figure S1, the spectra of TMSCN and phosphorane 1e was shown in (a) and (b), respectively. The mixing of 1e and TMSCN in a 1:2 ratio resulted a new peak at 126.07 ppm (c), suggesting the interaction of 1e and TMSCN leading to the polarization of Si-CN bond. When the ratio further increased to 1:1 and 2:1, this peak further shifted to 127.43 (d) and 127.88 ppm (e), respectively, possibly due to the formation of penta- or hexacoordinated silicon species. On the other hand, the signals of the C1 and C2 carbon of phosphorane 1e also had obvious change: The characteristic peak of C1 shifted from 172.10 to 171.81, 171.83, 171.86 ppm, while that of C2 slightly shifted from 41.33 to 41.42 and 41.43 ppm.

\section{Figure S1 Full ${ }^{13} \mathrm{C}$ NMR spectrum in $\mathrm{CD}_{3} \mathrm{CN}$}

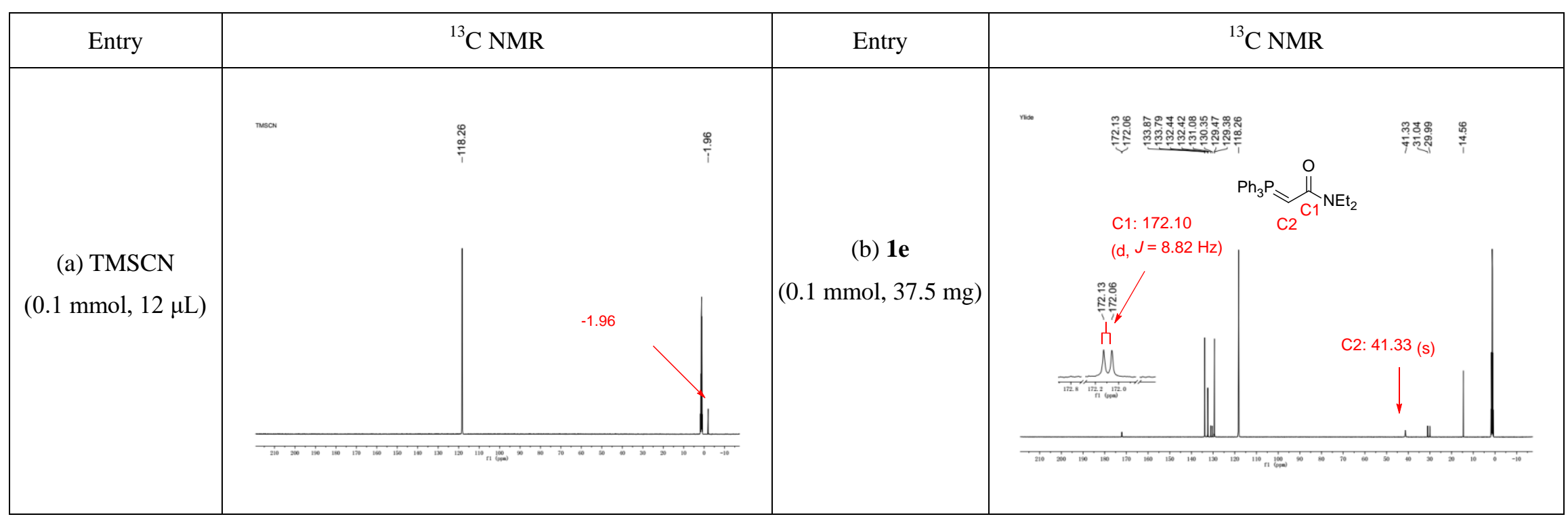




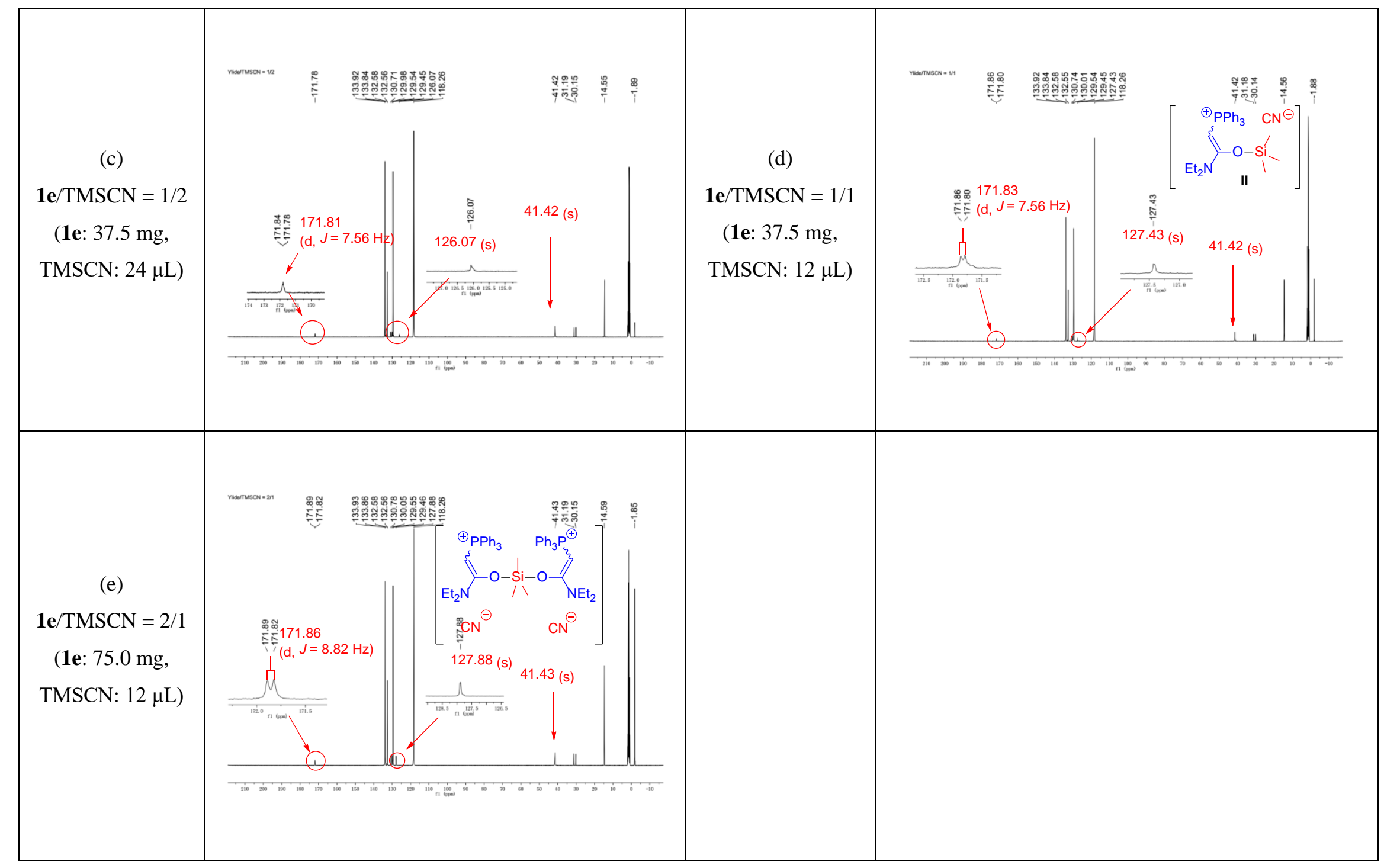




\section{1-2. In situ IR studies}

The in situ IR studies were conducted at room temperature using a $25 \mathrm{~mL}$ three-necked bottle, in which ReactIR $^{\mathrm{TM}} 15$ (by METTLER TOLEDO) and its fiber probe were used. After setting up the apparatus, $2.5 \mathrm{~mL}$ of $\mathrm{CH}_{2} \mathrm{Cl}_{2}$ was first added into the three-necked bottle and balanced for 10 min before the slow addition of the corresponding compounds for in situ IR study.

\section{1-2-1. IR spectrum of 1a and 1e}

The purpose of React IR analysis was to further probe the activation of TMSCN by phosphorus ylides. Before the analysis of the interaction of ylides and TMSCN, we measured the IR spectrum of ylide 1a and 1e (Figure S2), but found two weak peaks 2071 and $2072 \mathrm{~cm}^{-1}$ on the zoomed spectra of 2000-2200 $\mathrm{cm}^{-1}$, respectively, which might overlap with characteristic peak of the isocyanide species resulting from the activation of TMSCN by ylides, as that of the isocyanide species $\mathrm{Ph}_{3} \mathrm{P}(\mathrm{OTMS})\left(\mathrm{N}=\mathrm{C}:\right.$ ) was detected to be $2072 \mathrm{~cm}^{-1}$ by Corey et al. Fortunately, the ester substituted ylide 1d was found to be suitable for the analysis, see section 1-2-2.

\section{Figure S2 IR spectrum of 1a and 1e}
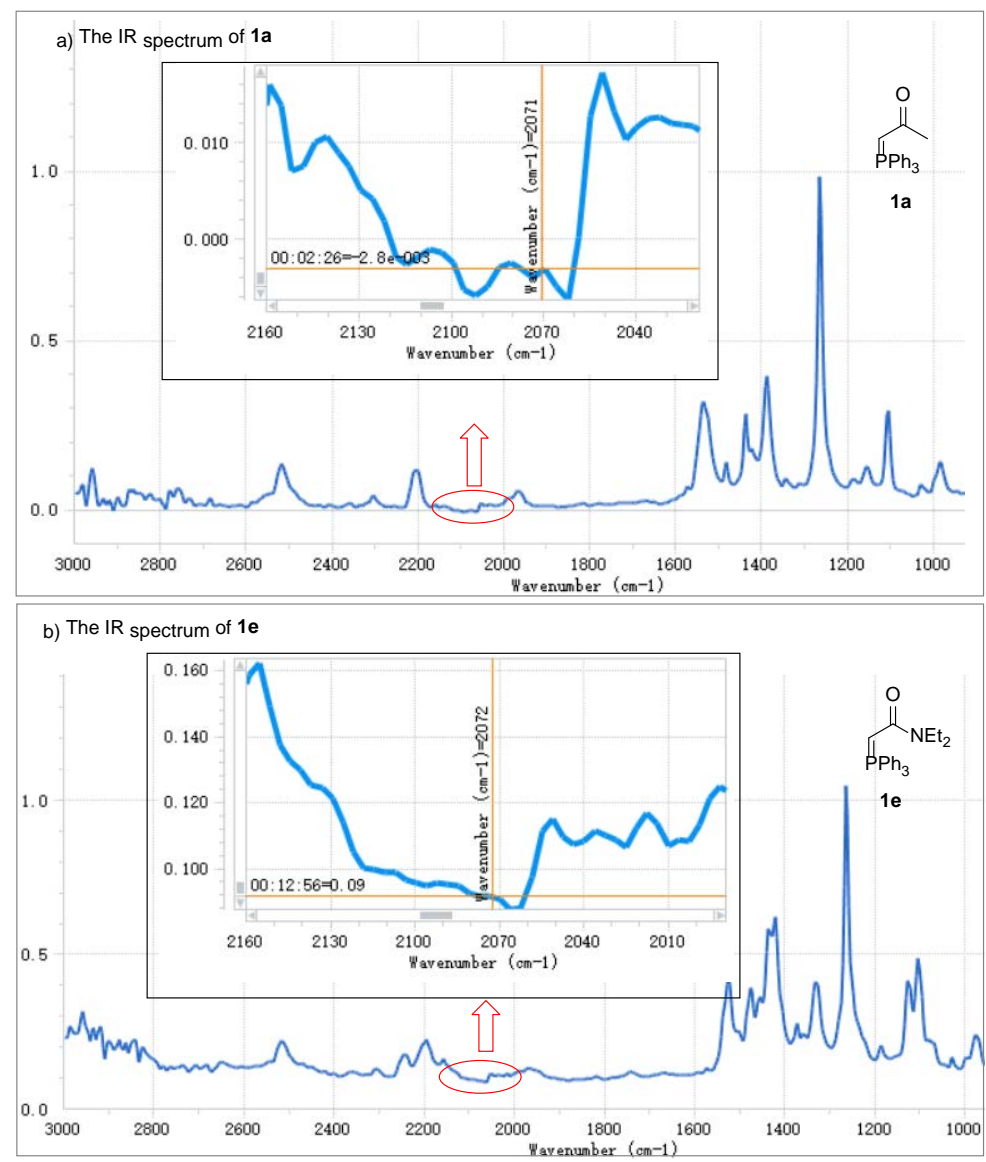


\section{1-2-2. IR study the activation of 1d to TMSCN}

Corey and Ryn have reported the IR analysis of a mixture of TMSCN and $\mathrm{Ph}_{3} \mathrm{PO}{ }^{1}$ They observed a new -NC stretching band at $2072 \mathrm{~cm}^{-1}$, different from that of TMSCN $\left(2192 \mathrm{~cm}^{-1}\right)$ and TMSNC (2095 $\mathrm{cm}^{-1}$ ), which suggested the formation of reactive isocyanide species $\mathrm{Ph}_{3} \mathrm{P}(\mathrm{OTMS})(\mathrm{N}=\mathrm{C}$ :).

In our IR analysis, ylide 1d (2.5 mmol) was dissolved in $\mathrm{CH}_{2} \mathrm{Cl}_{2}(2.5 \mathrm{~mL})$ (Figure S3). After $10 \mathrm{~min}$, TMSCN (5.0 mmol) was added slowly, and then the mixture of TMSCN and ylide 1d showed a new -NC stretching band at $2073 \mathrm{~cm}^{-1}$. This was in agreement with Corey and Ryn's finding. When TMSCN was increased to $0.4 \mathrm{mmol}$, this peak reached its highest. With the continuous addition of TMSCN, this peak no longer rise, reaching to an equilibrium. Furthermore, the absorption peak of 1d obviously decreased at $2051 \mathrm{~cm}^{-1}$, suggesting the interaction between $1 \mathbf{d}$ and TMSCN.

\section{Figure S3 IR Study the activation of 1d to TMSCN}

(a) React IR study of the reaction between $\mathbf{1 d}$ and TMSCN

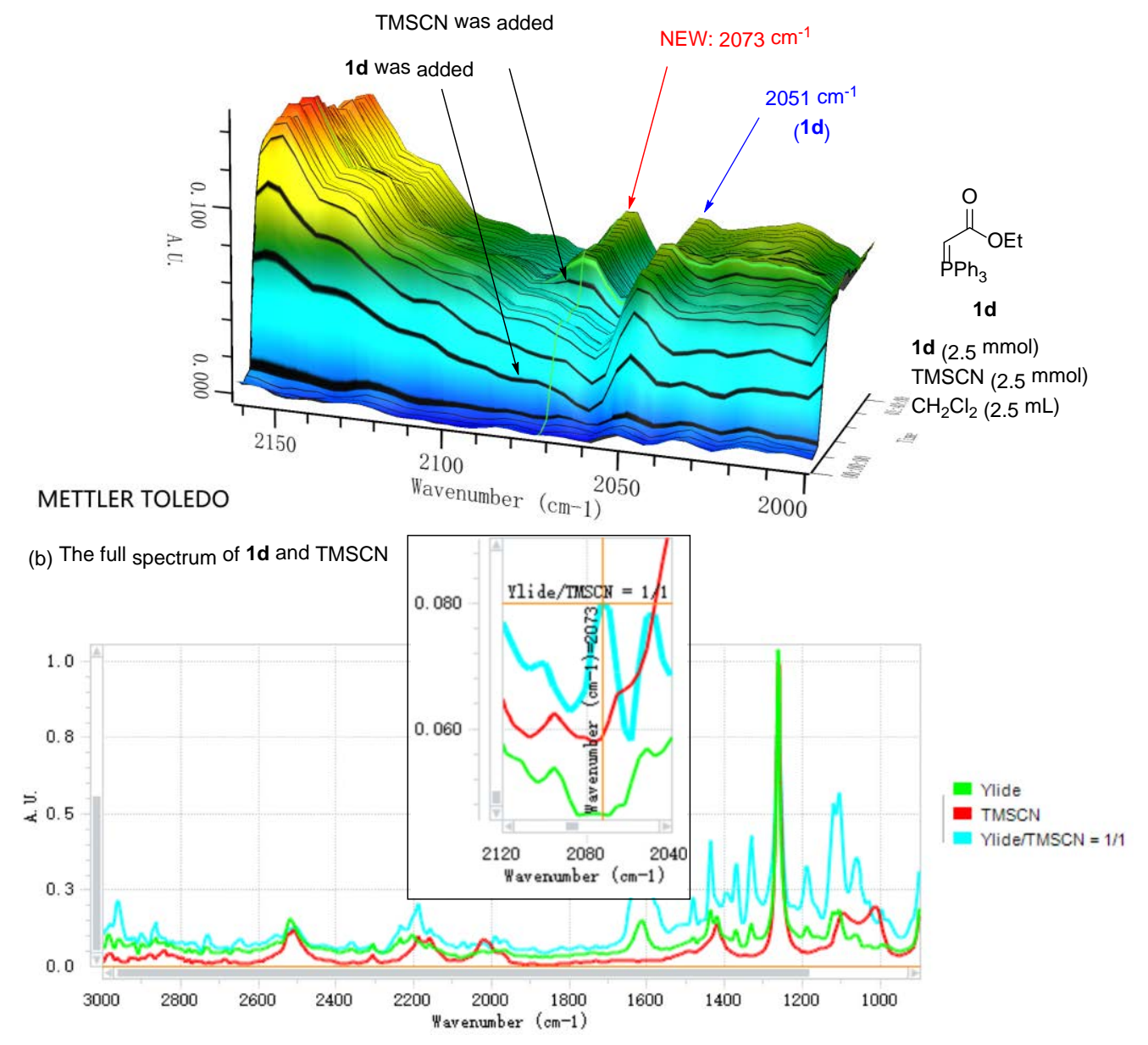

[1] Ryu, D. H.; Corey, E. J. Highly Enantioselective Cyanosilylation of Aldehydes Catalyzed by a Chiral Oxazaborolidinium Ion. J. Am. Chem. Soc. 2004, 126, 8106-8107. 
2. NMR spectra.

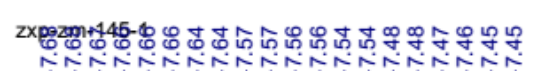

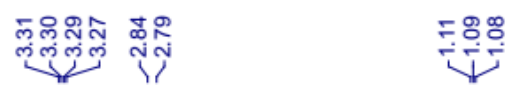

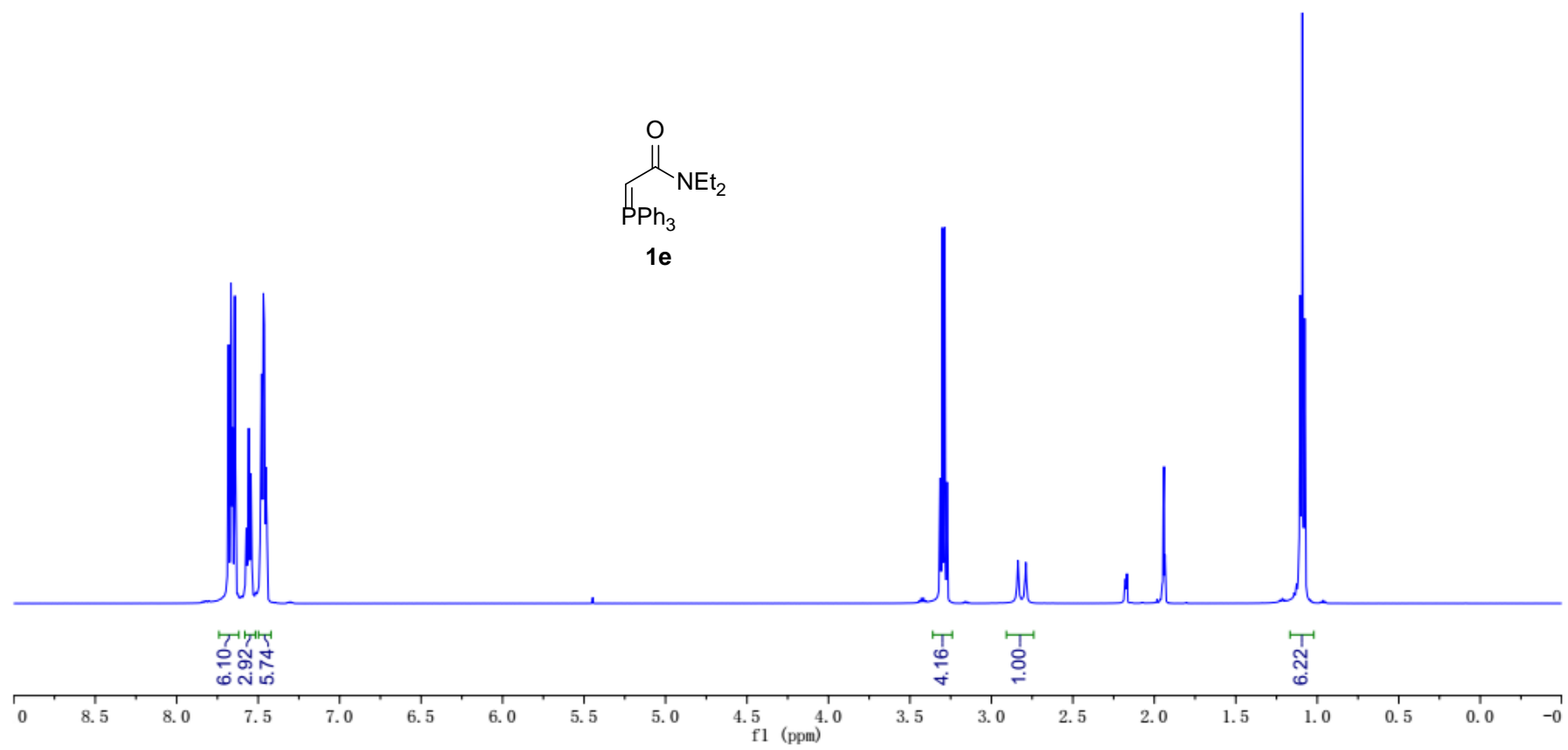



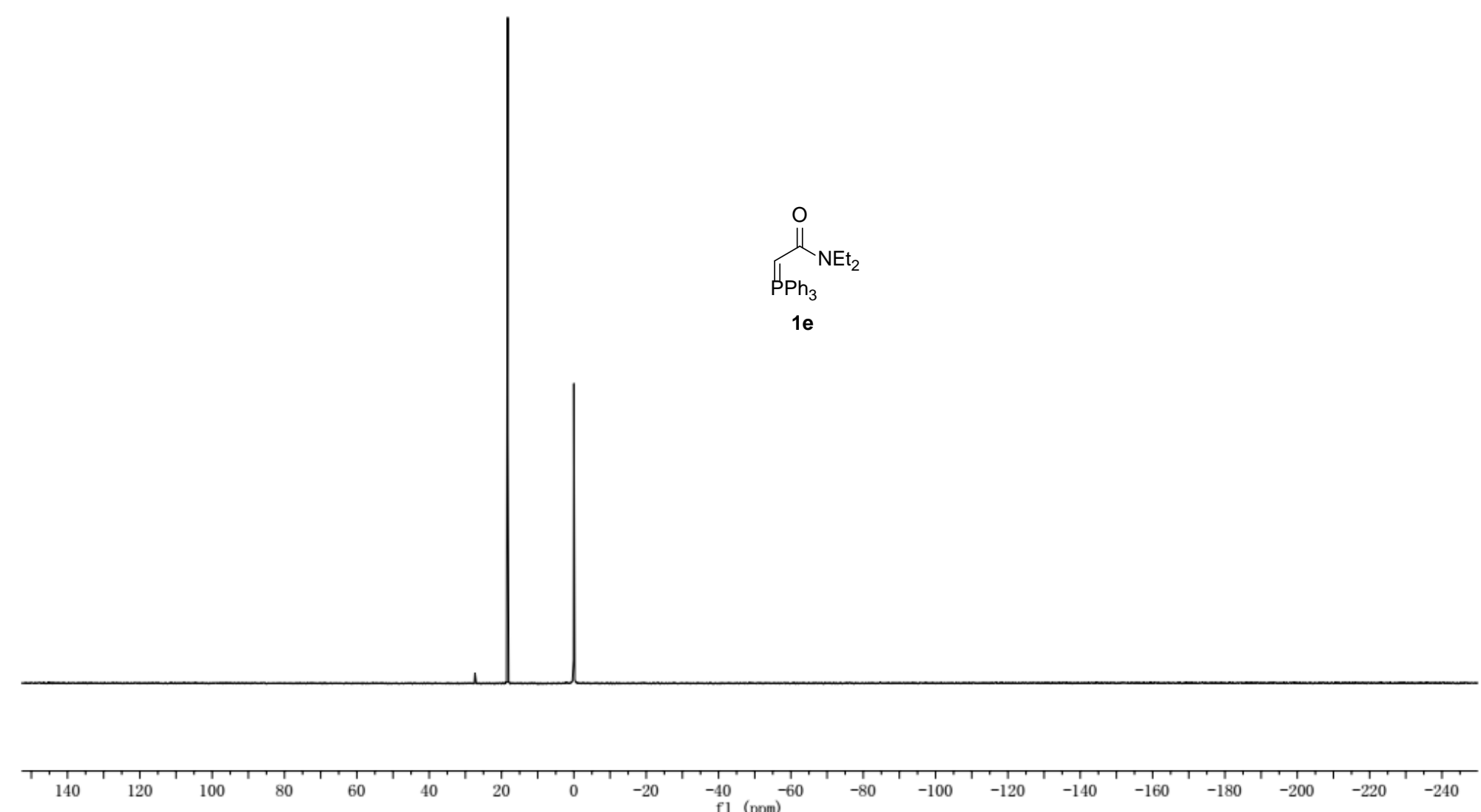


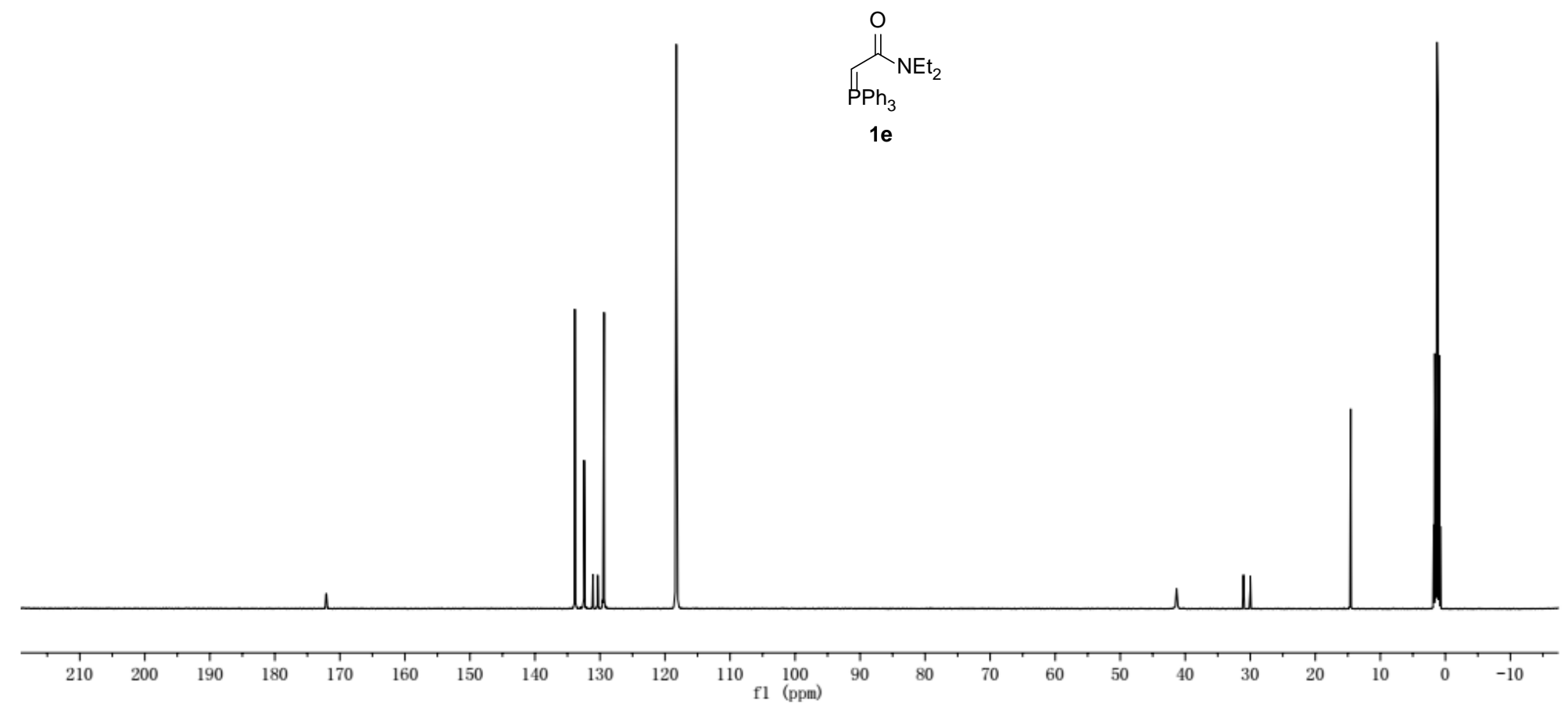




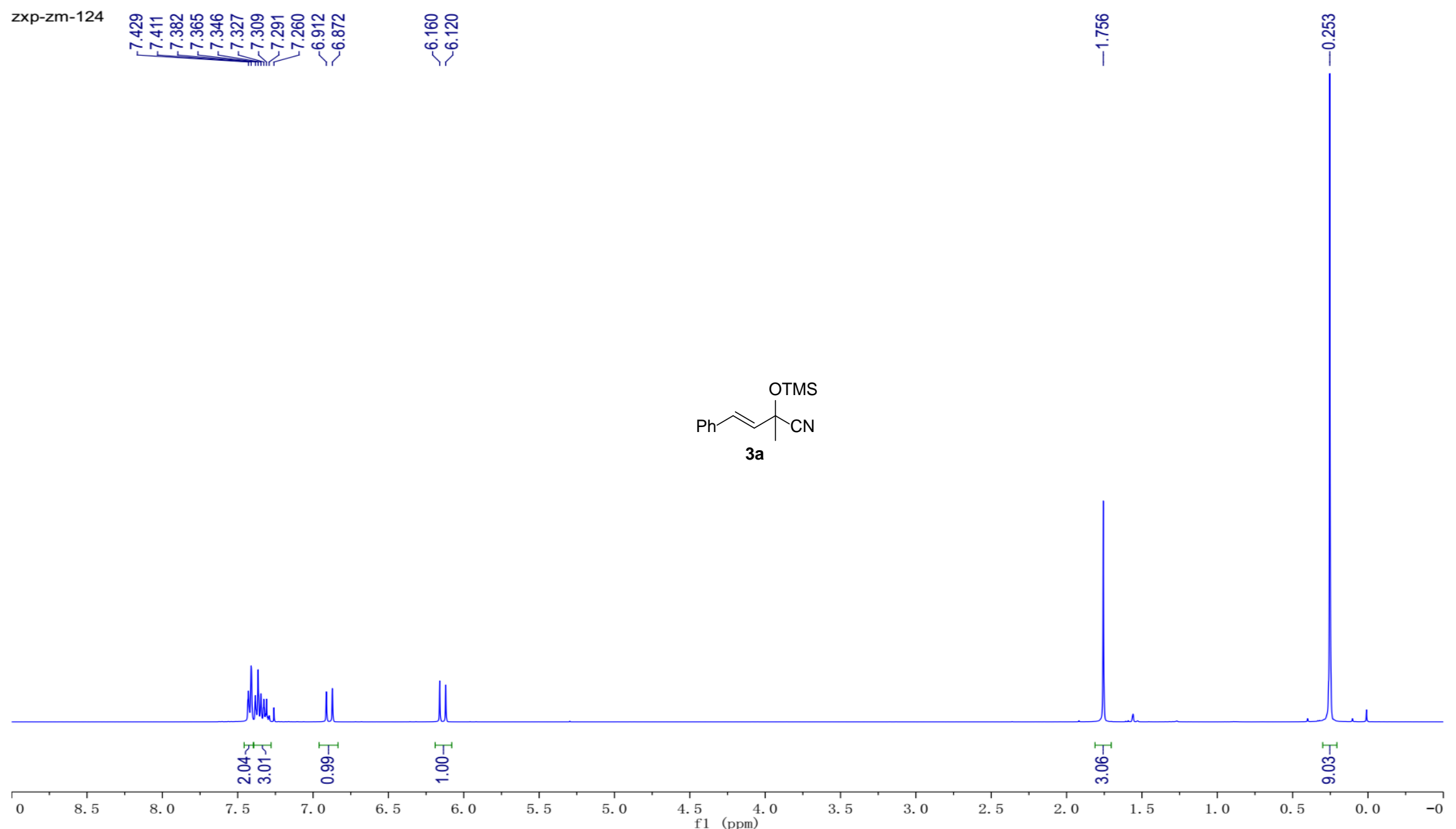


zxp-zm-124

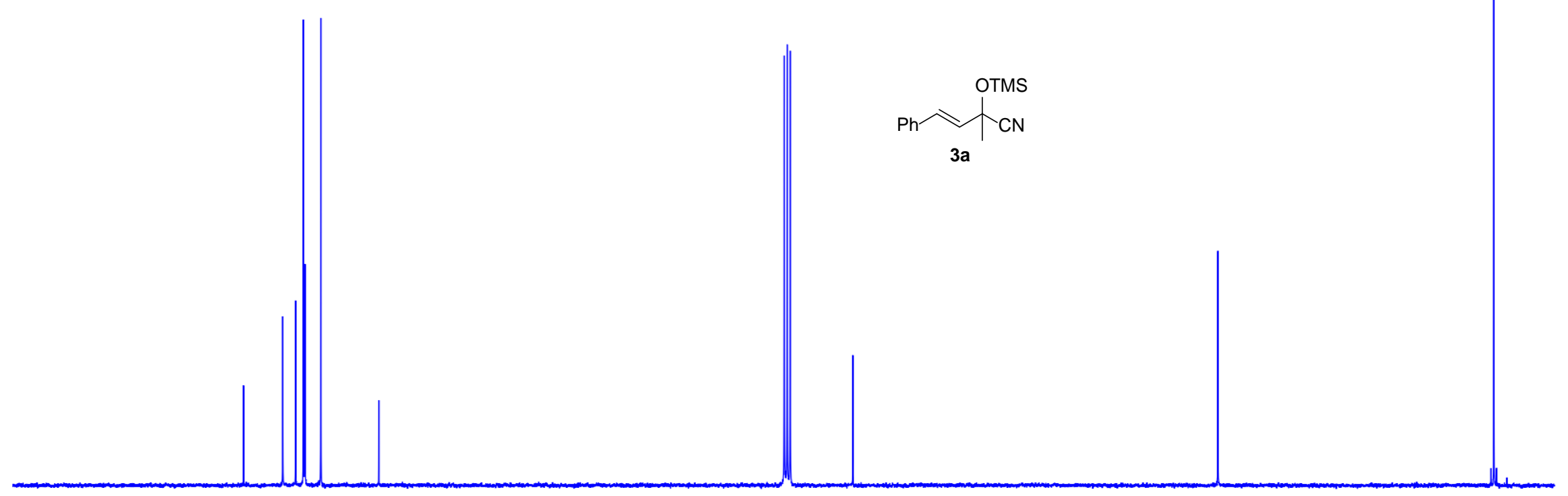

30 150 


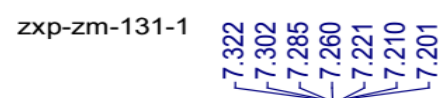

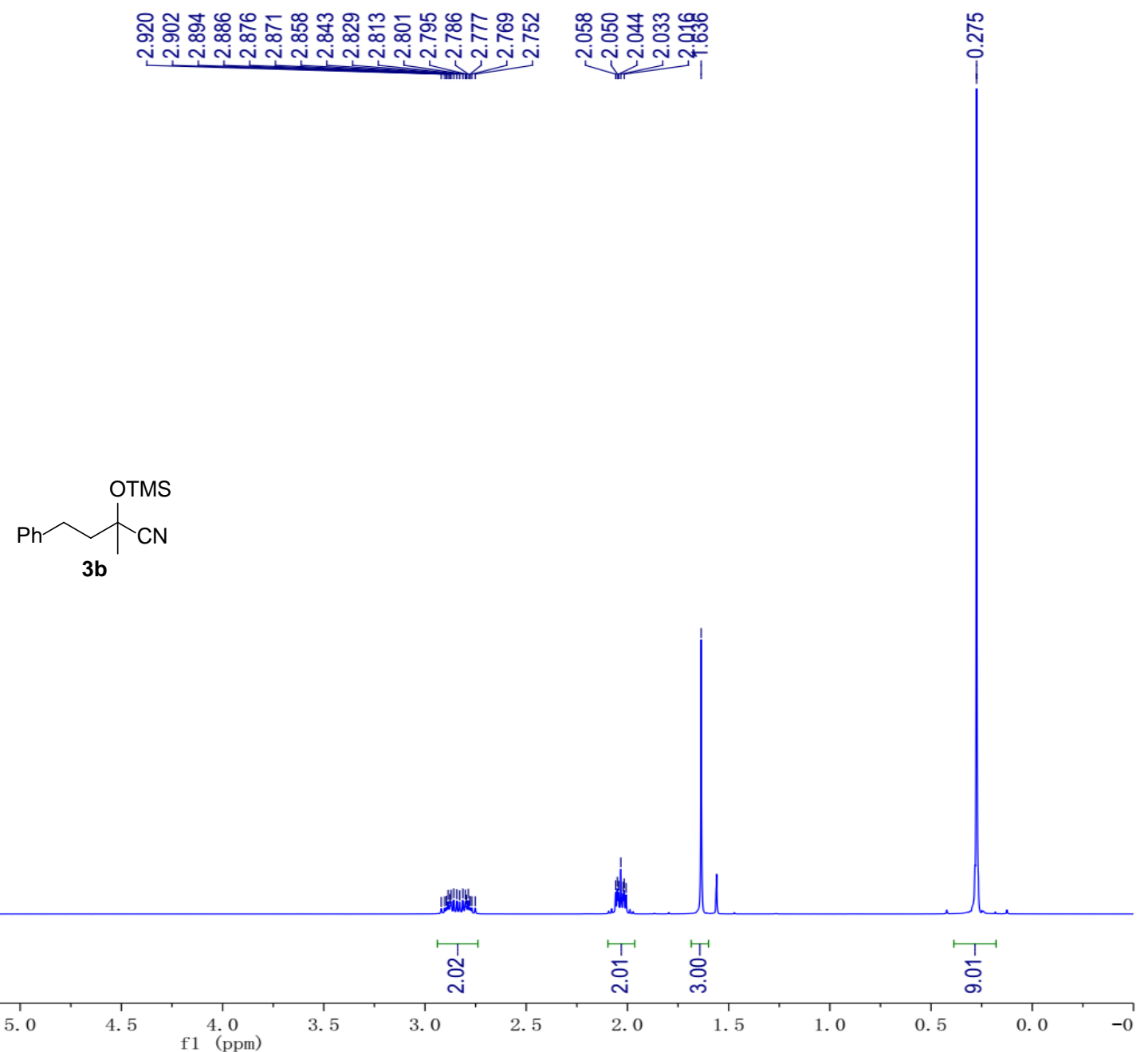




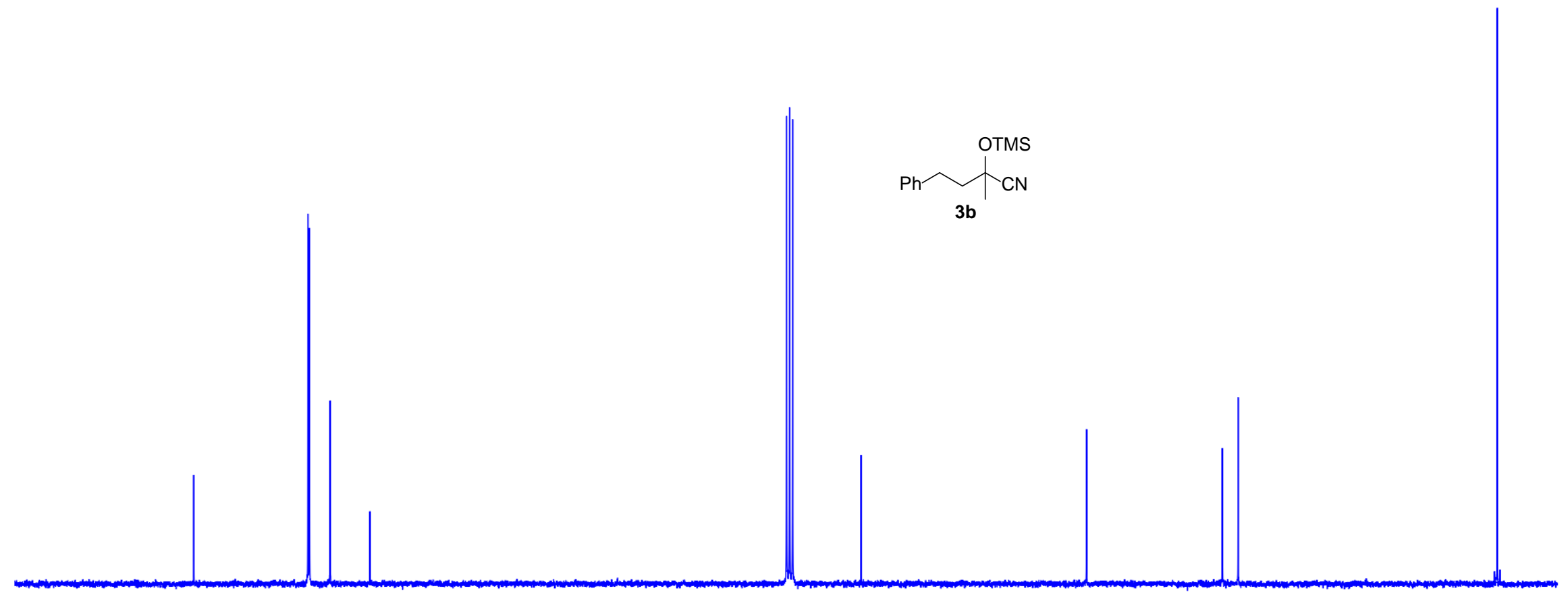

30 


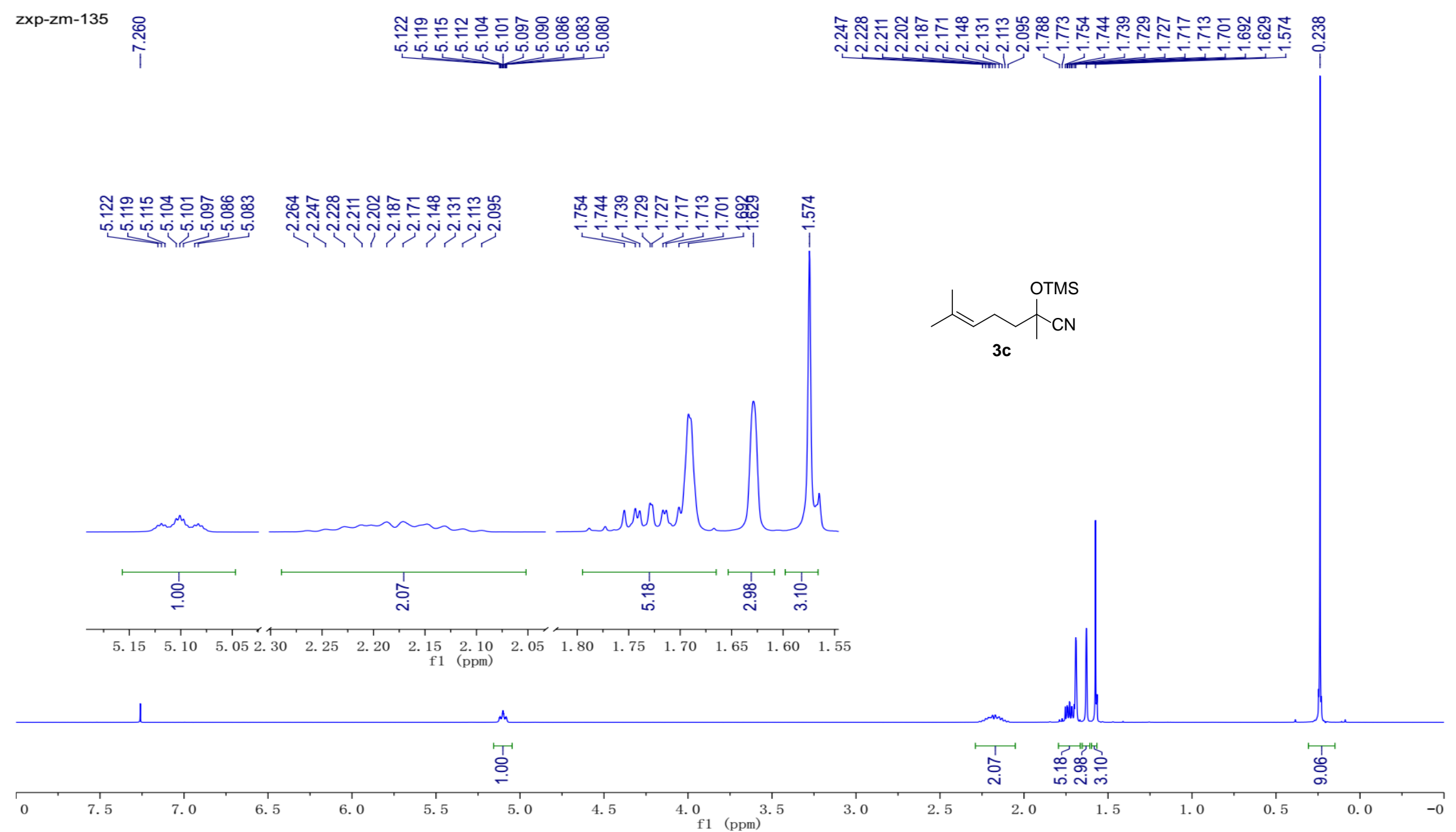


zxp-zm-135
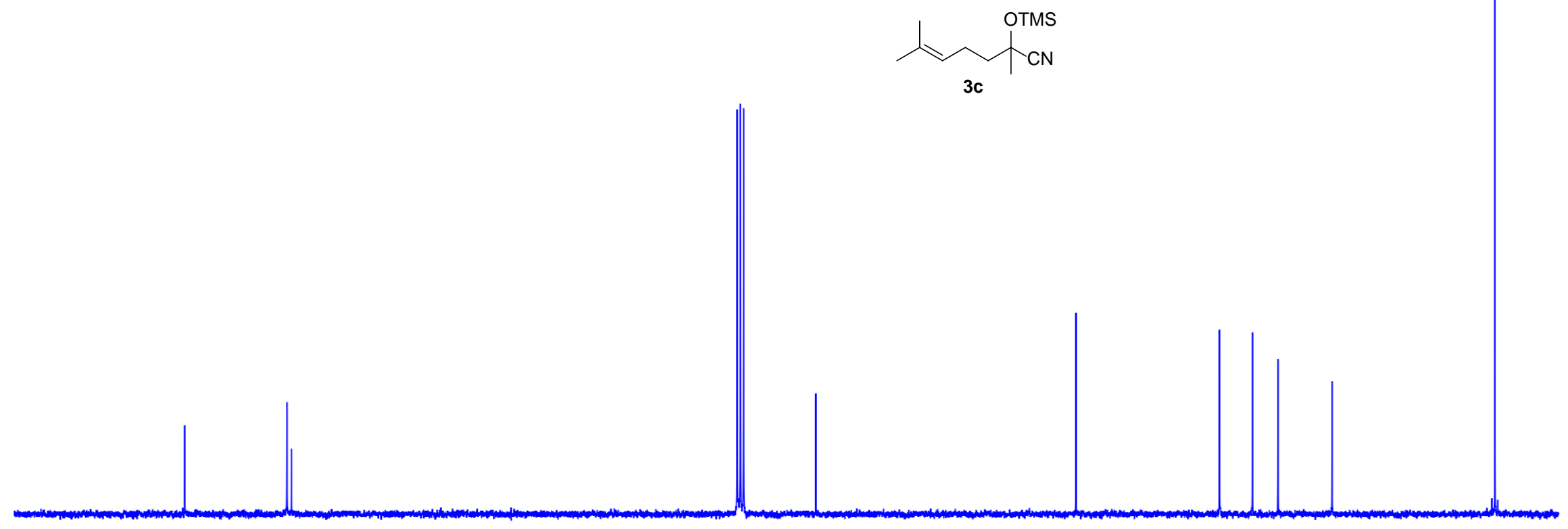

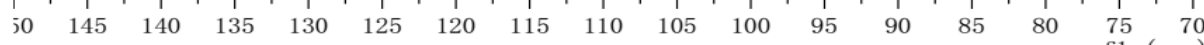

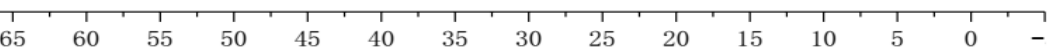




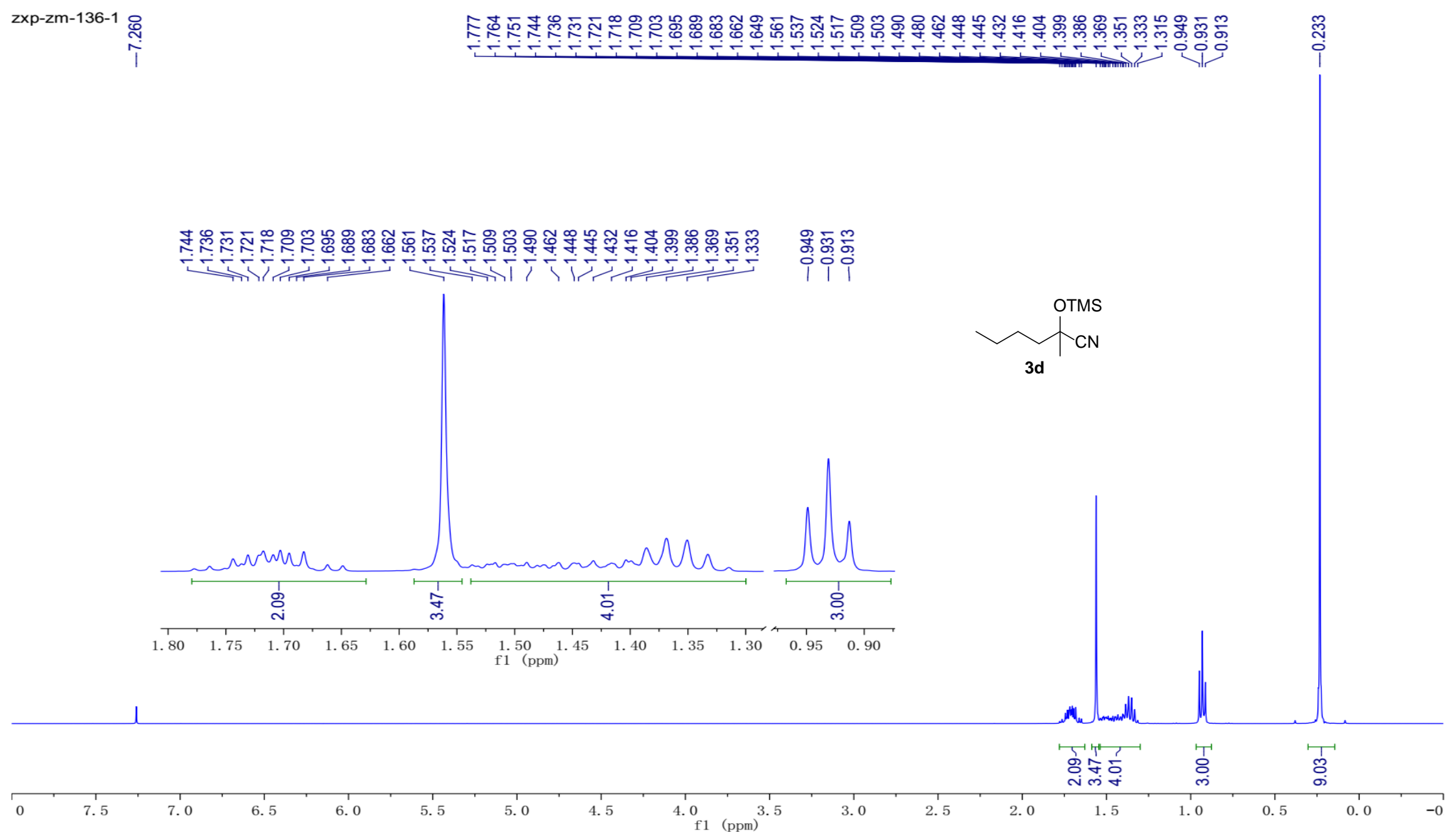


zxp-zm-136-1

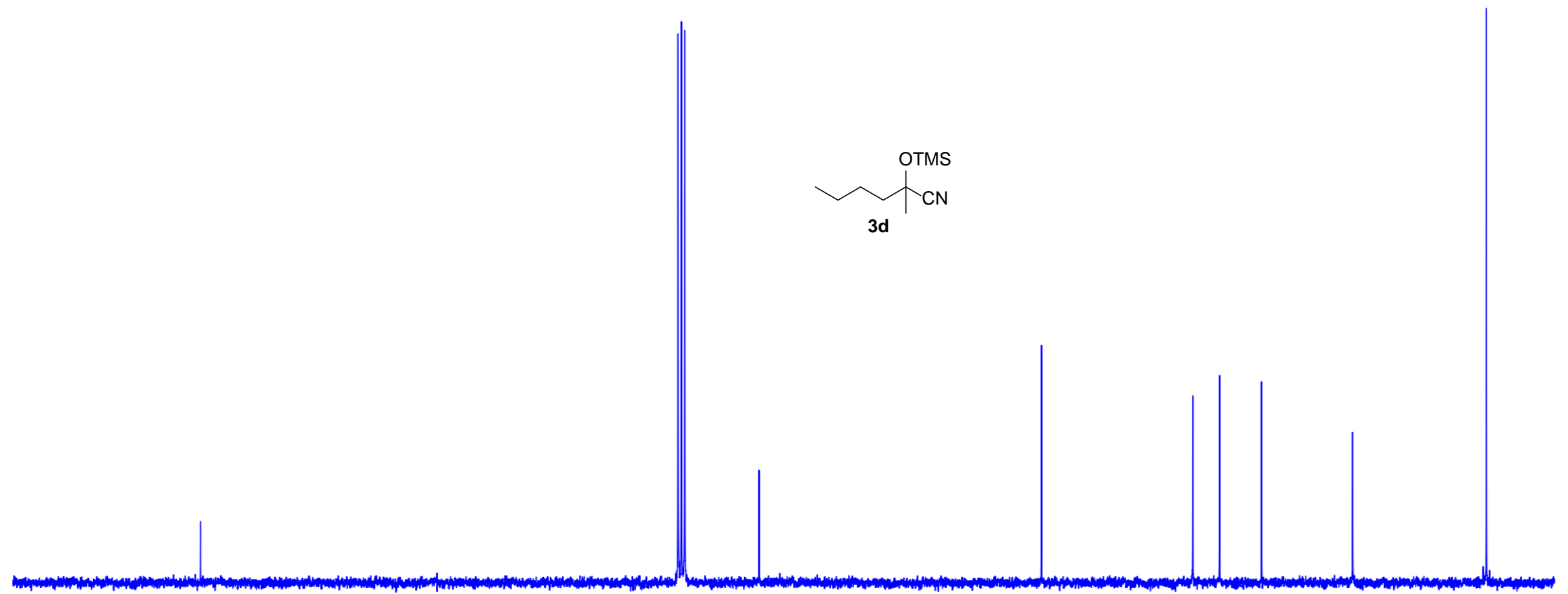

\begin{tabular}{lllllllllllllllll}
\hline 10 & 135 & 130 & 125 & 120 & 115 & 110 & 105 & 100 & 95 & 90 & 85 & 80 & 75 & 70 & 65
\end{tabular} 


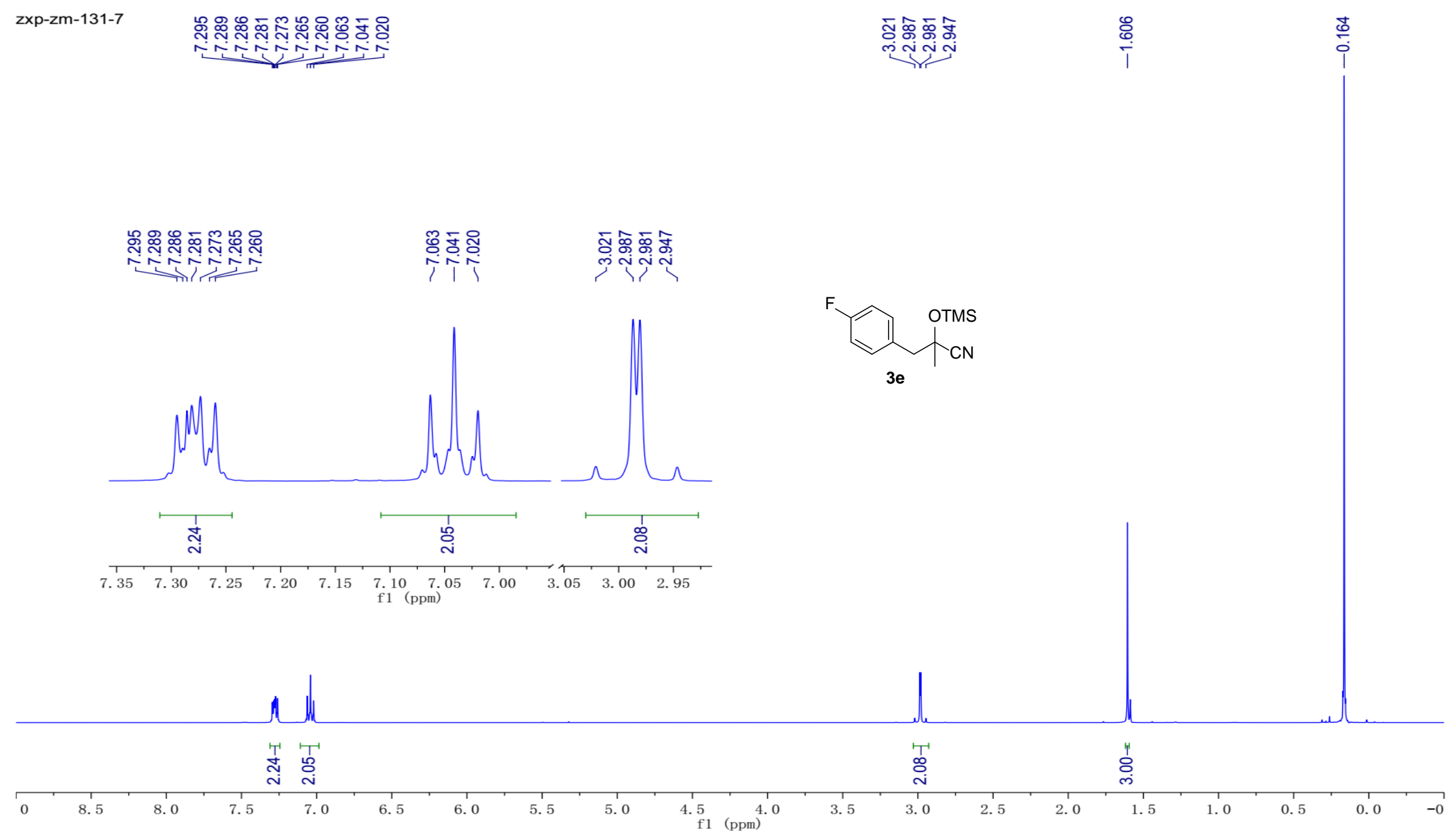


zxp-zm-131-7

li

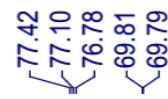

$\stackrel{\substack{+ \\ \text { i }}}{\text { I }}$

ष্.
iे
।

$\stackrel{8}{i}$

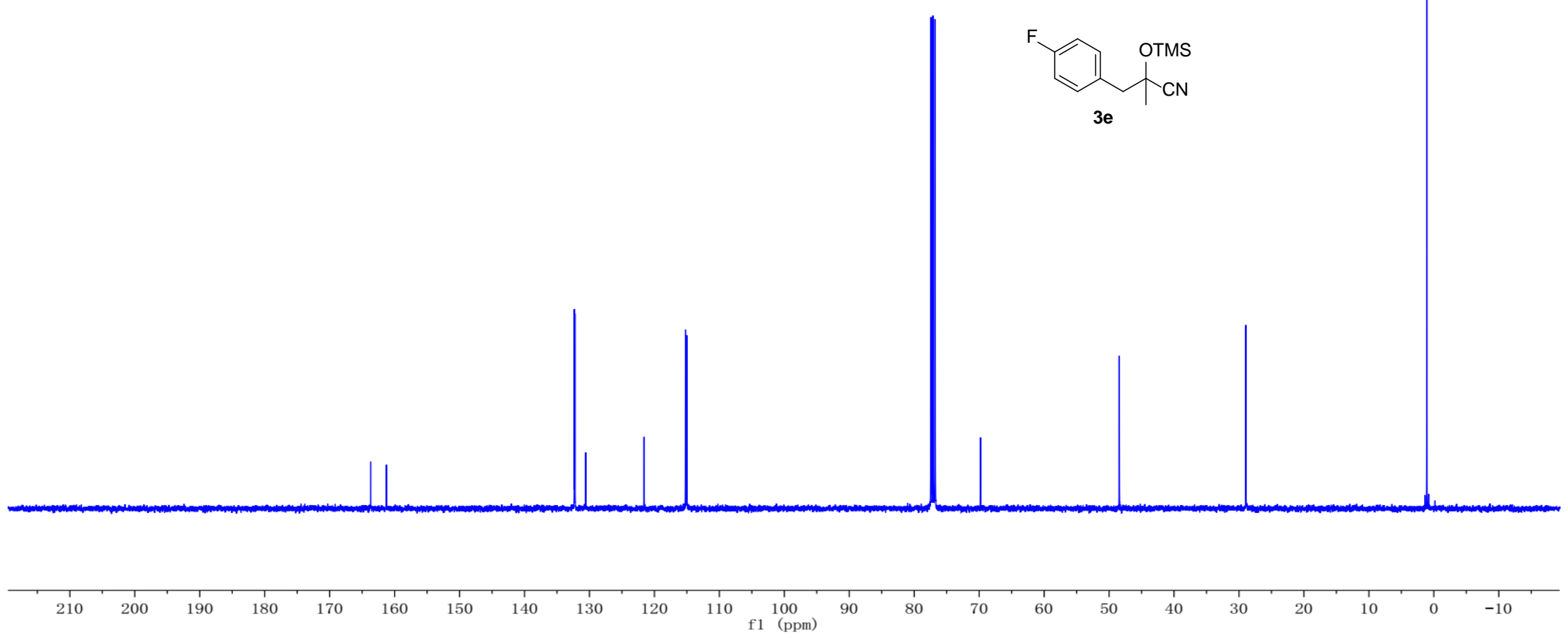

S18 
zxp-zm-131-7

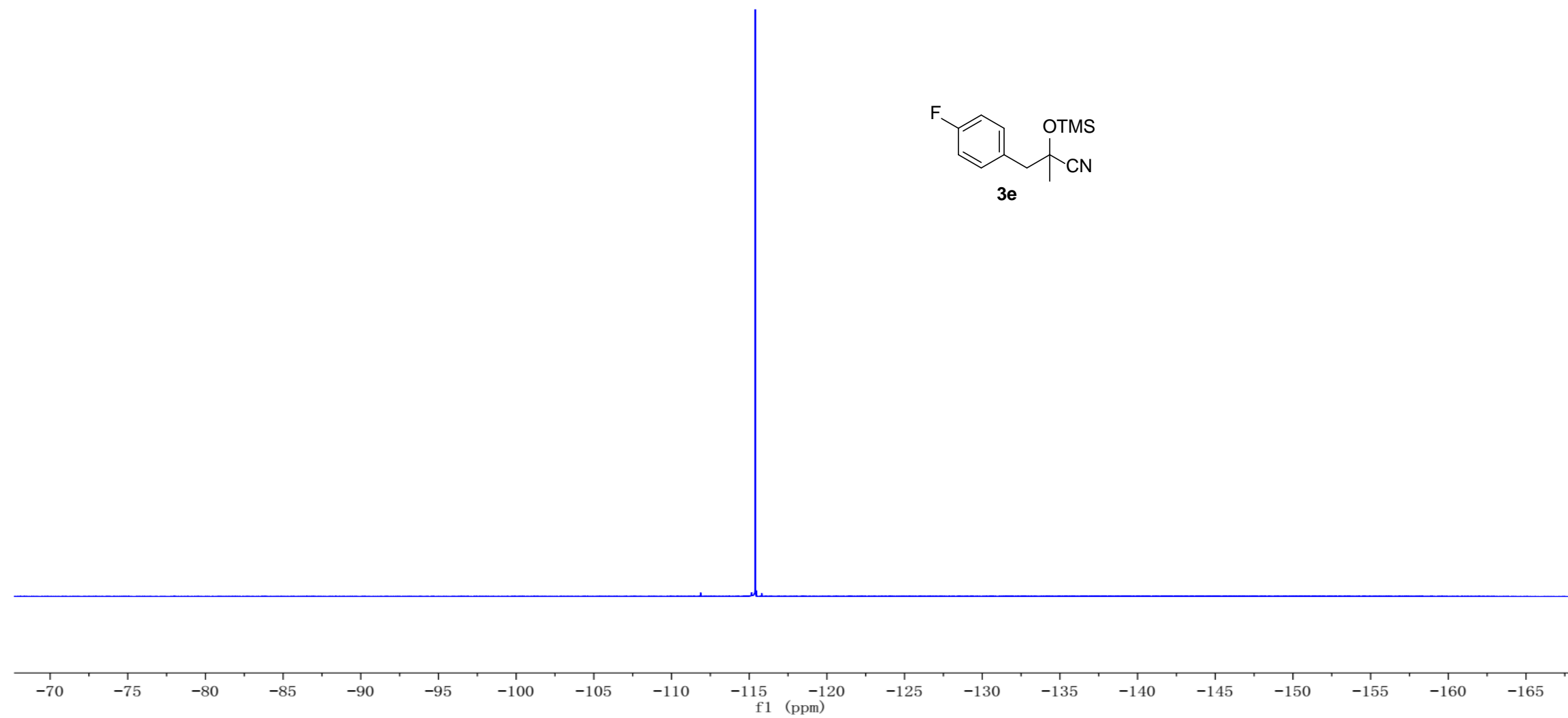




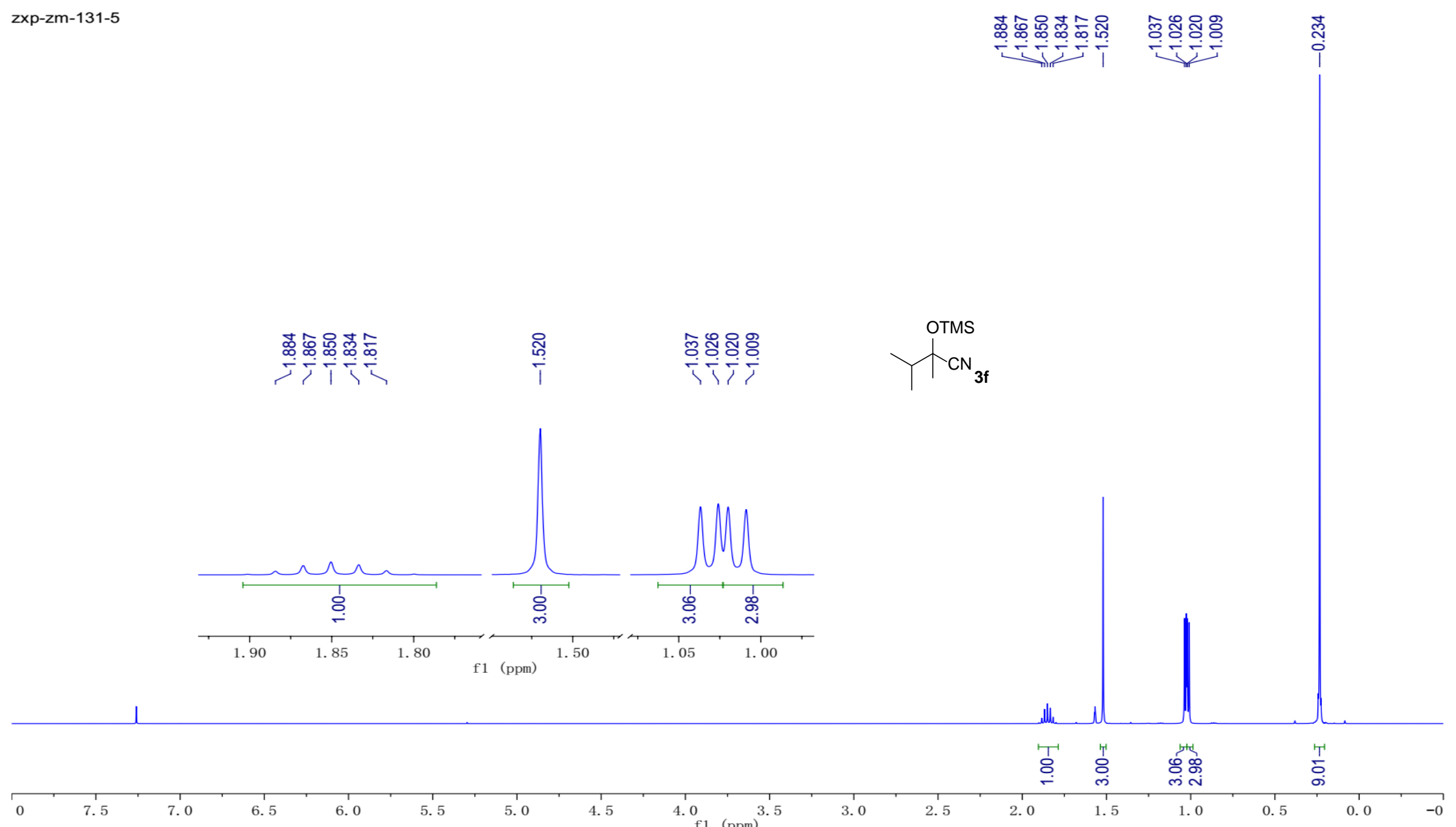


zxp-zm-131-5

구은

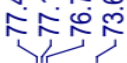

กั๊

$\stackrel{\bar{m}}{\Gamma}$

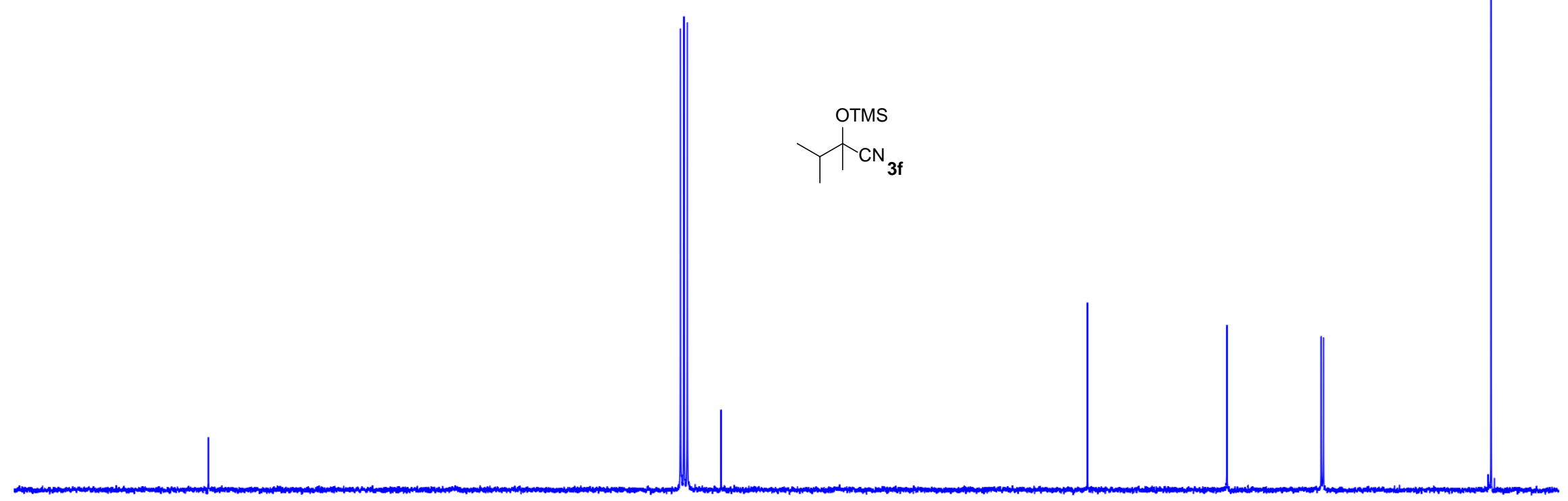

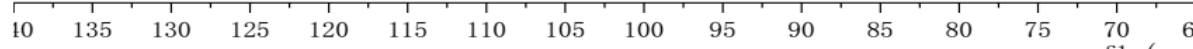




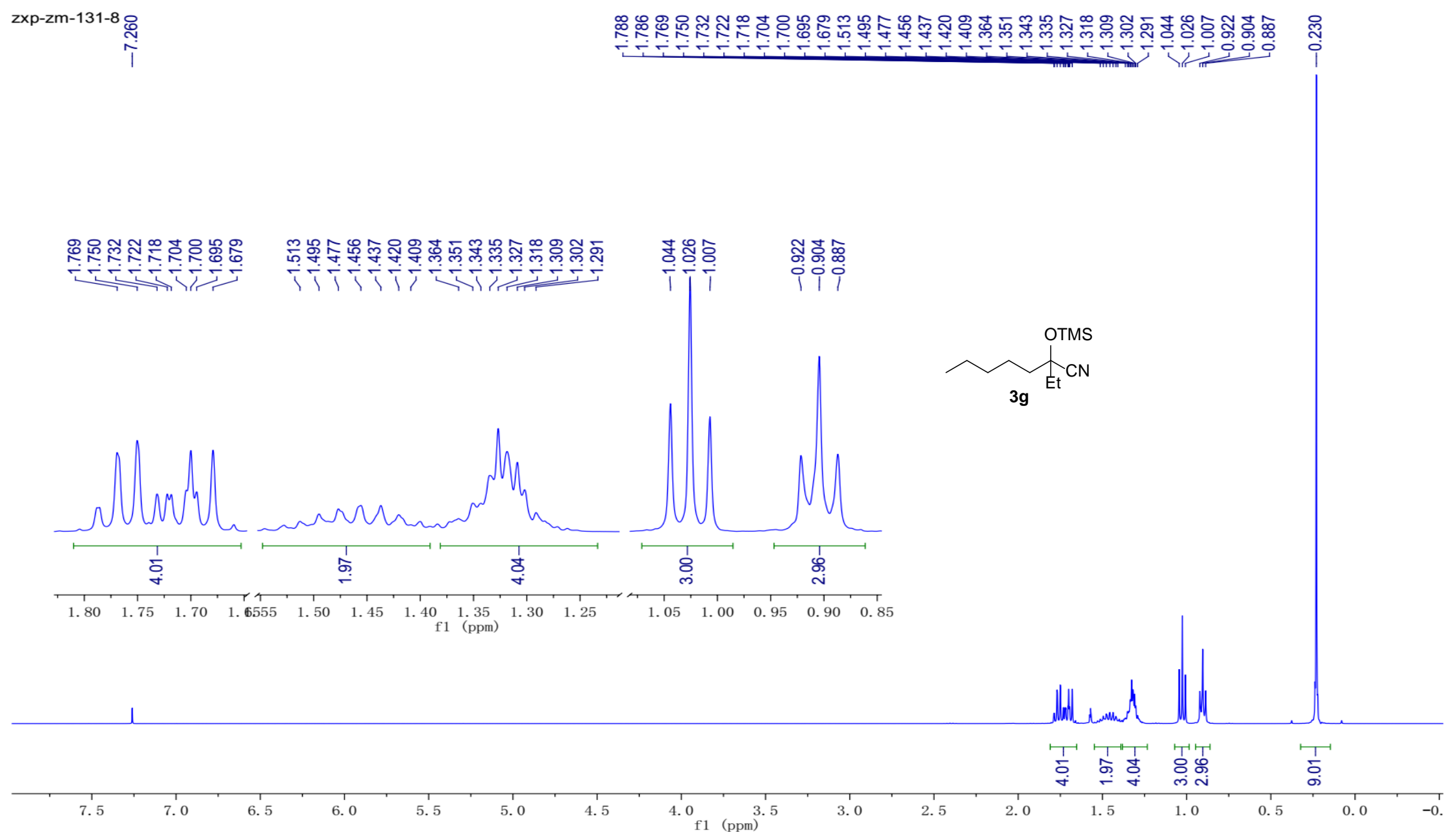


zxp-zm-131-8

ํํㅇำ

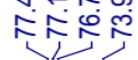

กิ

กู่

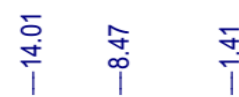

OTMS

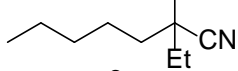

$3 g$

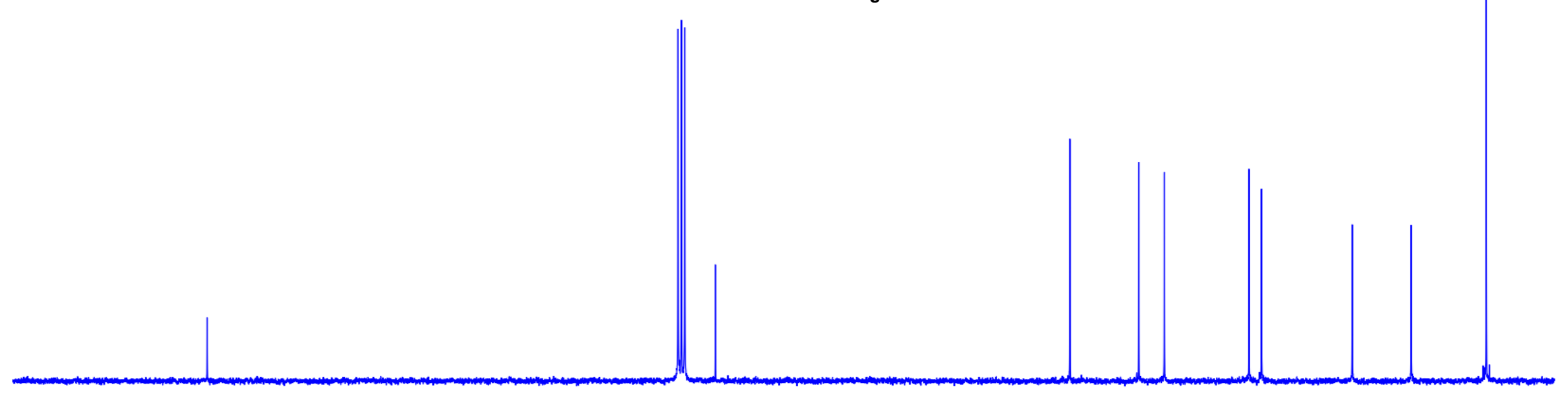

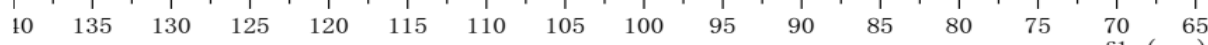



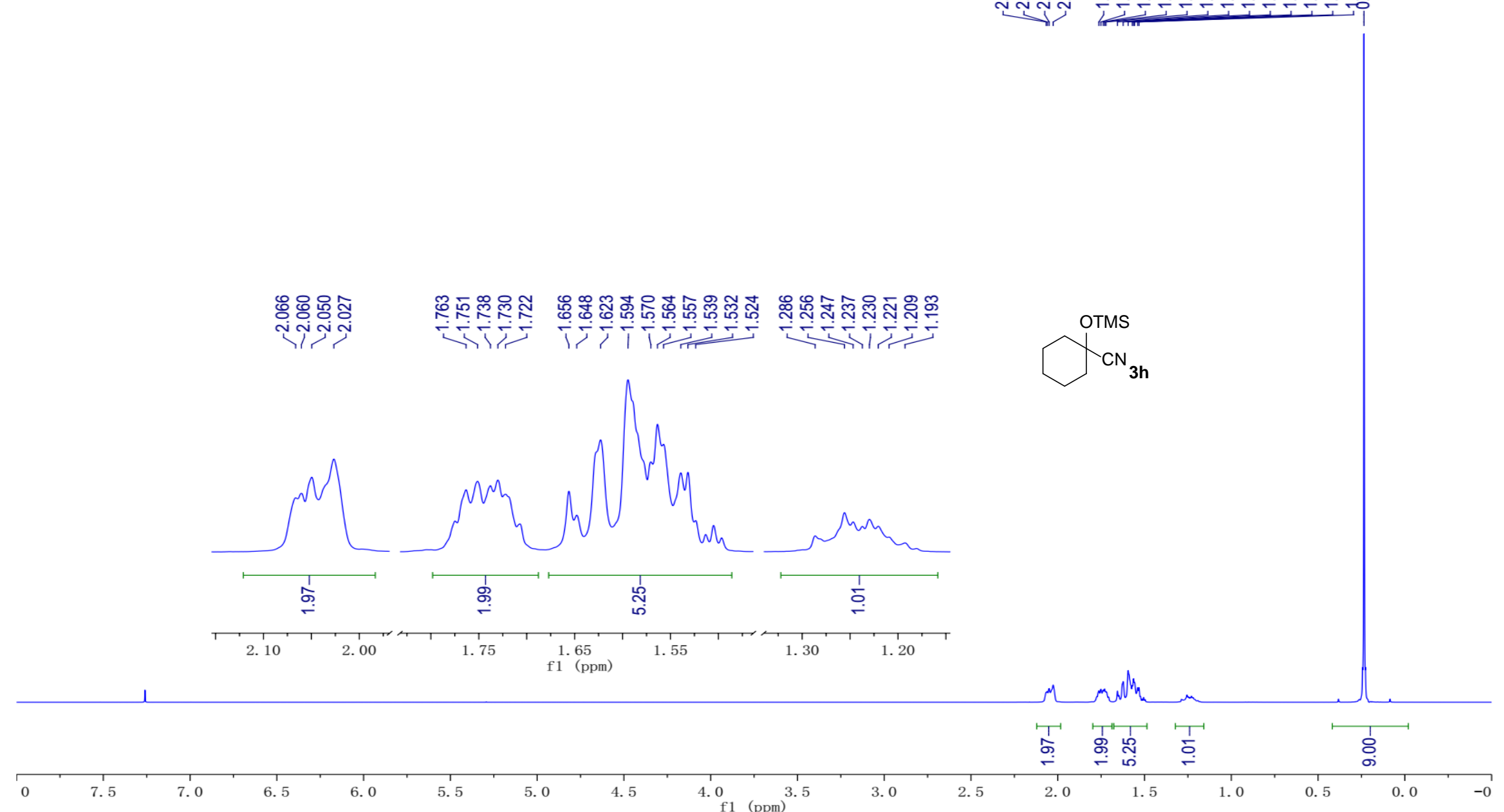


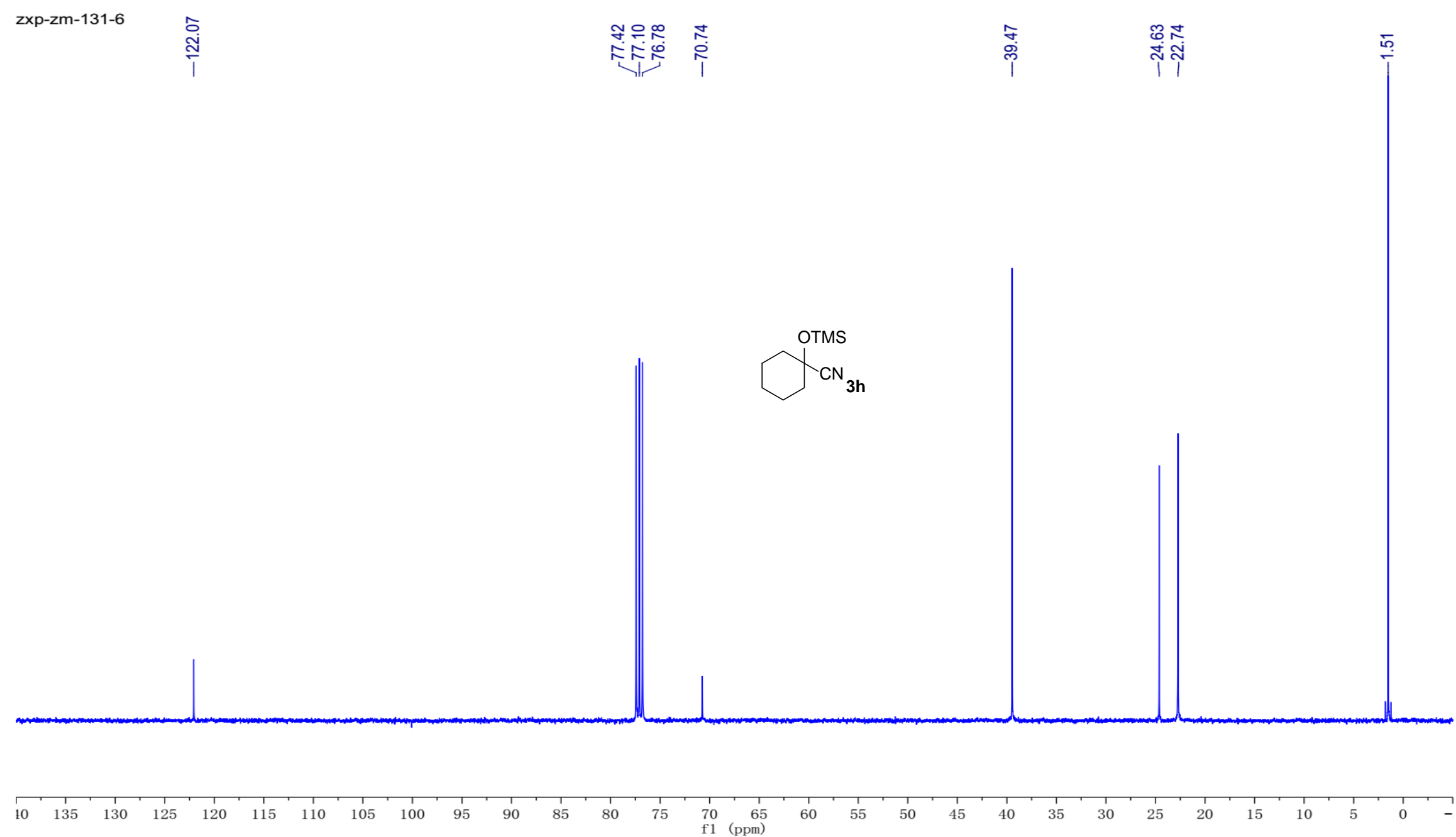




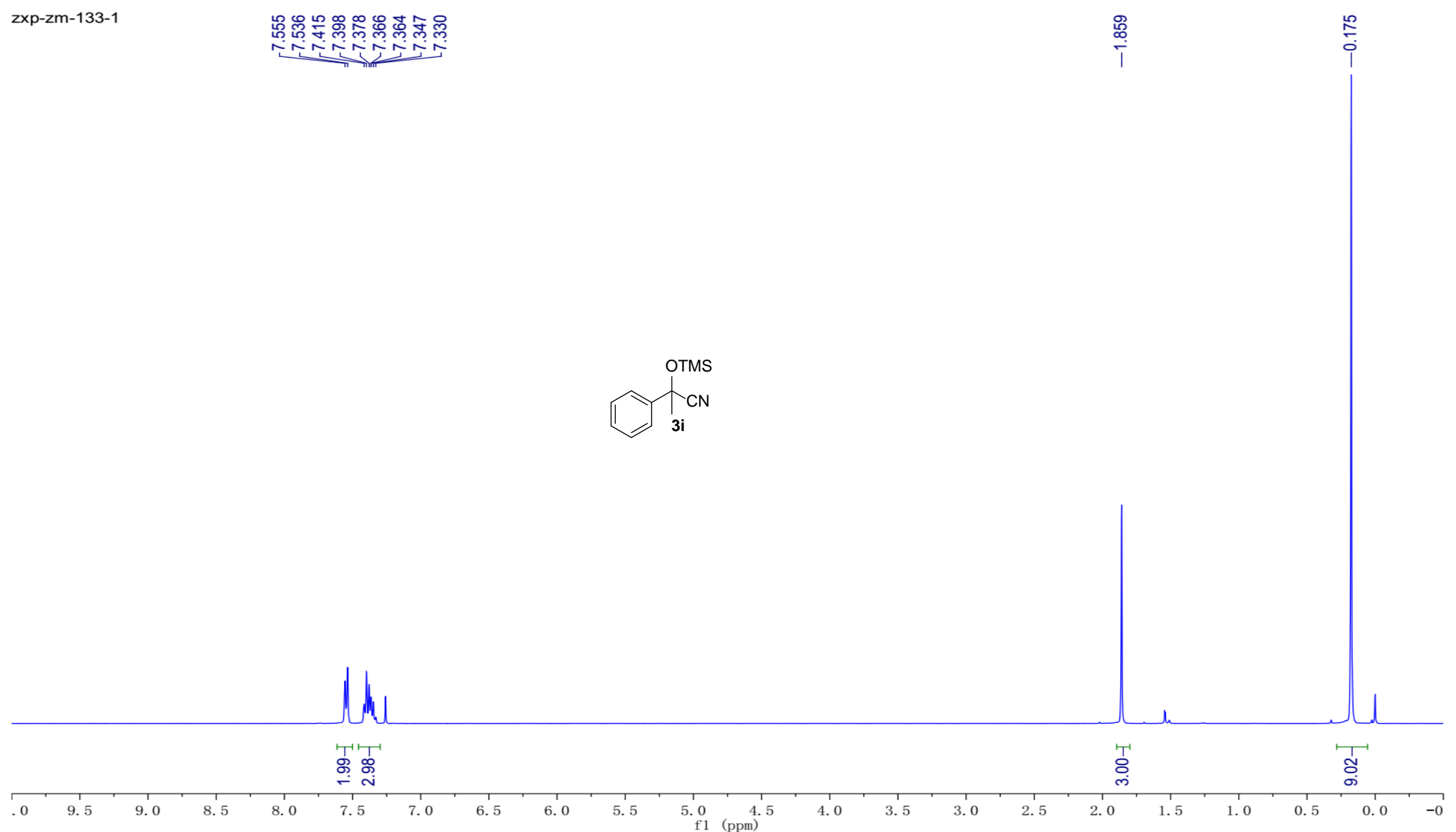


zxp-zm-133-1

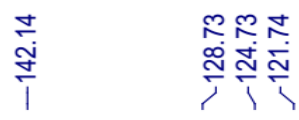

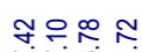

순유

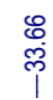

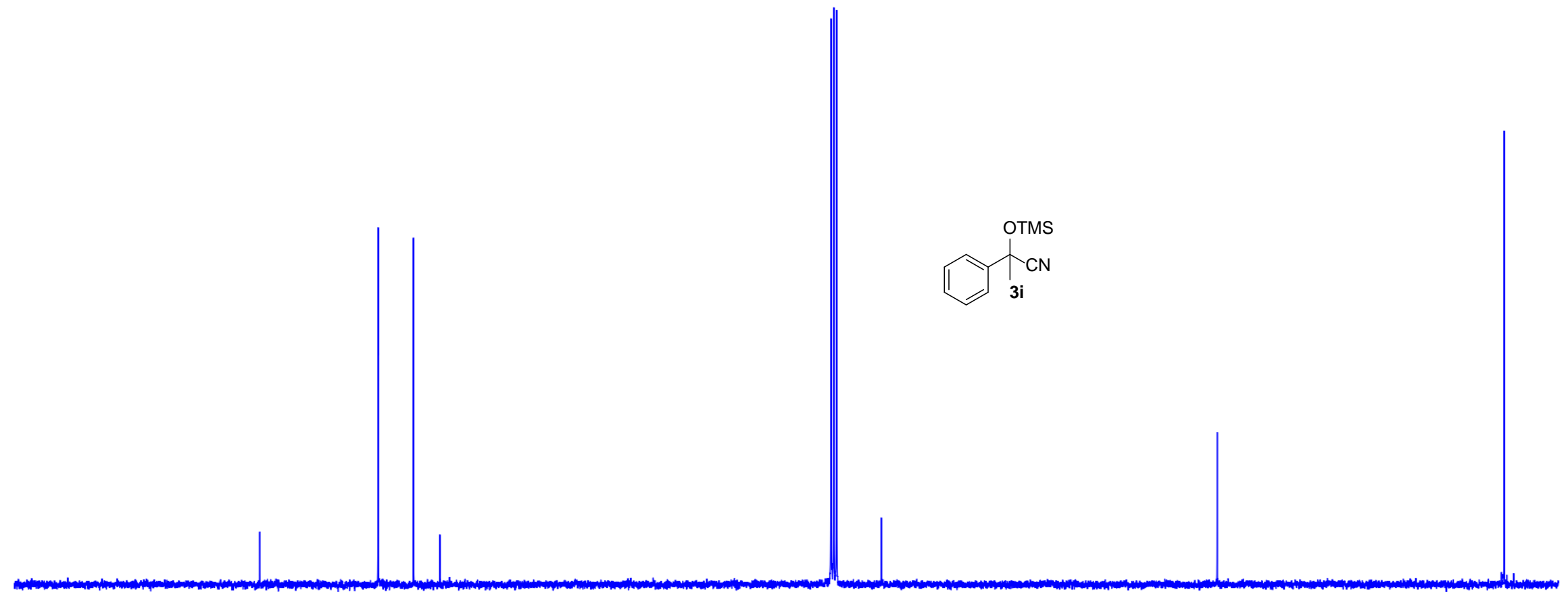

70 160

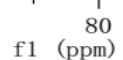




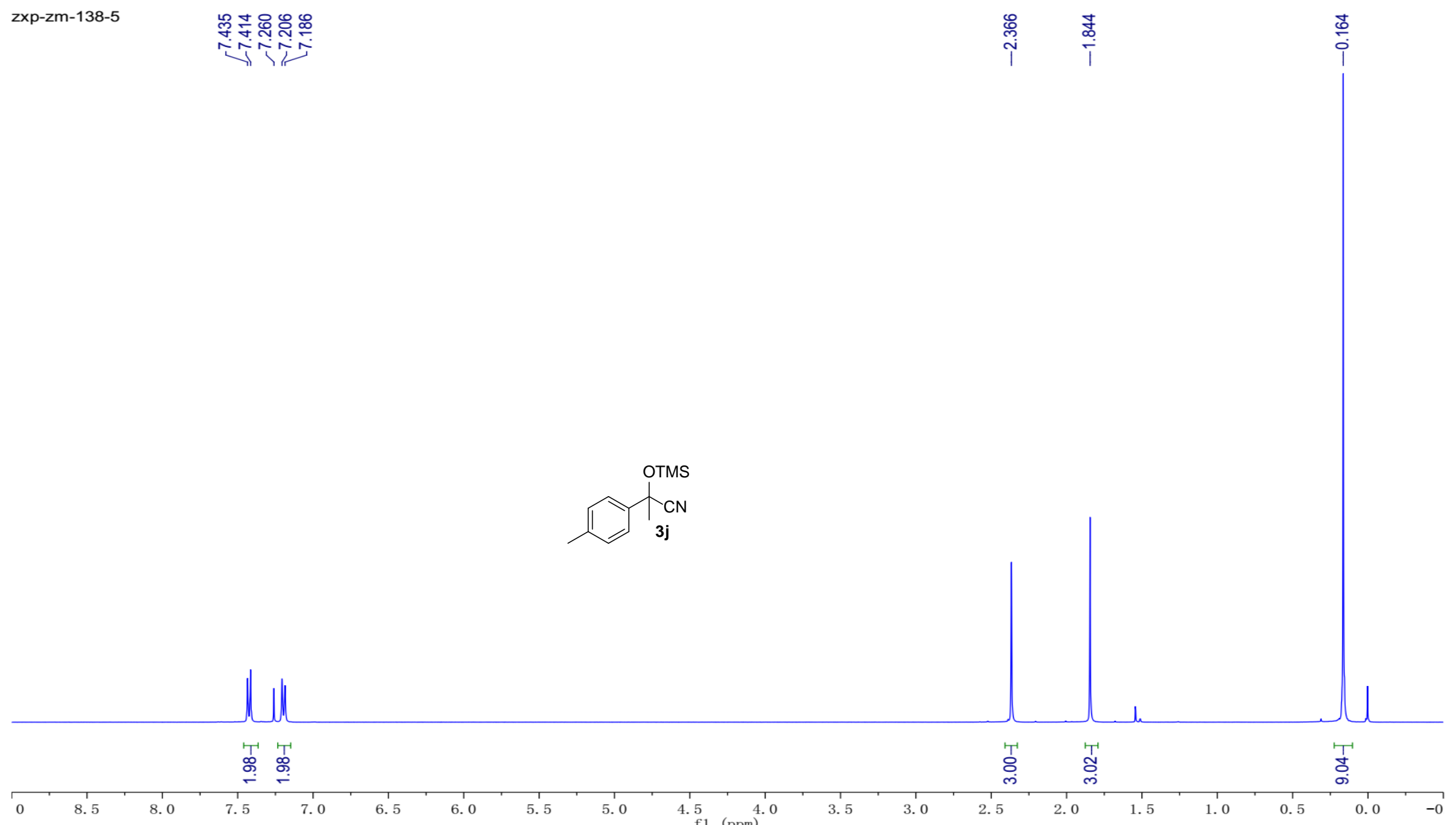



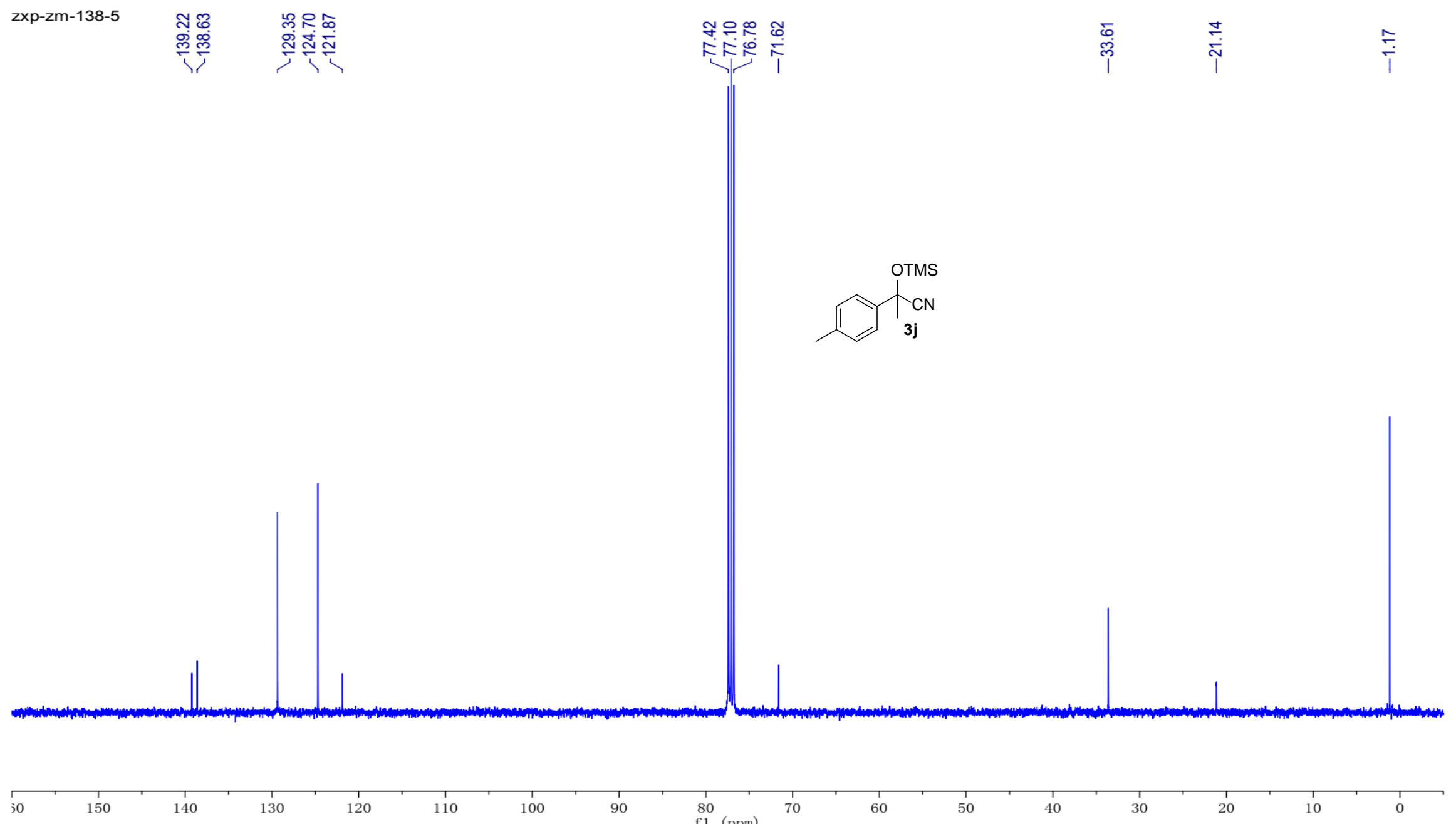
zxp-zm-133-4
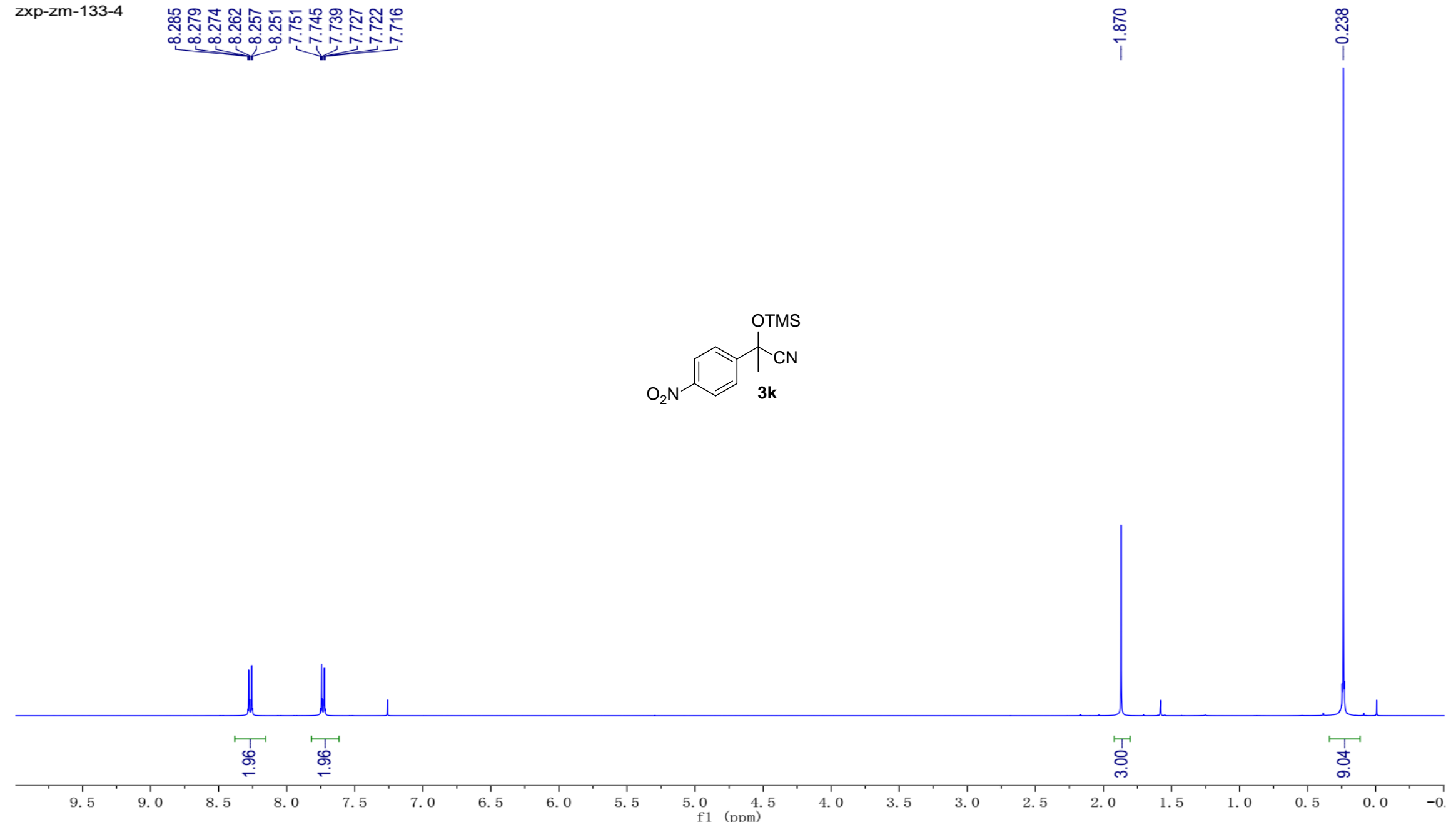

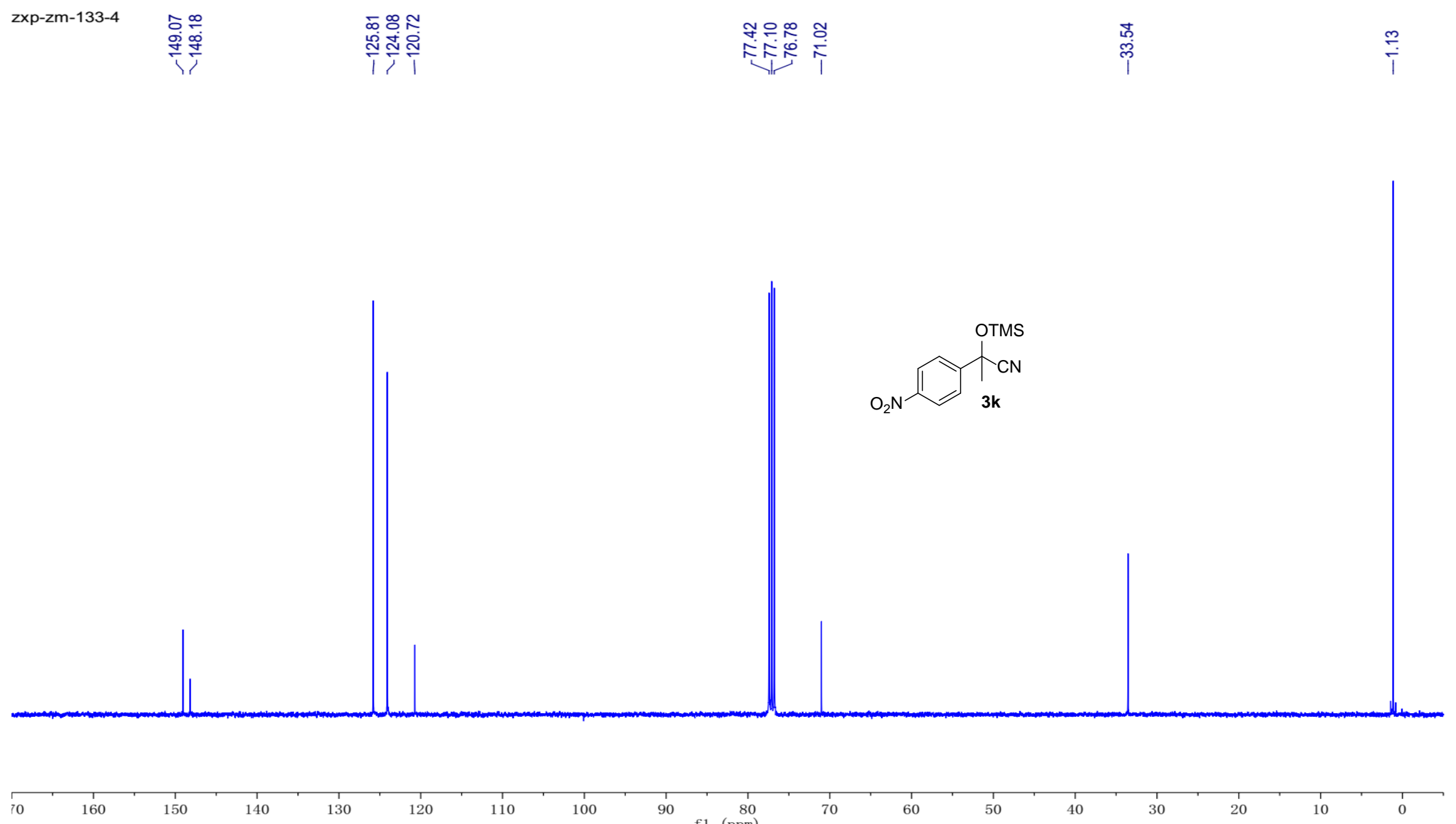
zxp-zm-133-3

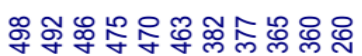
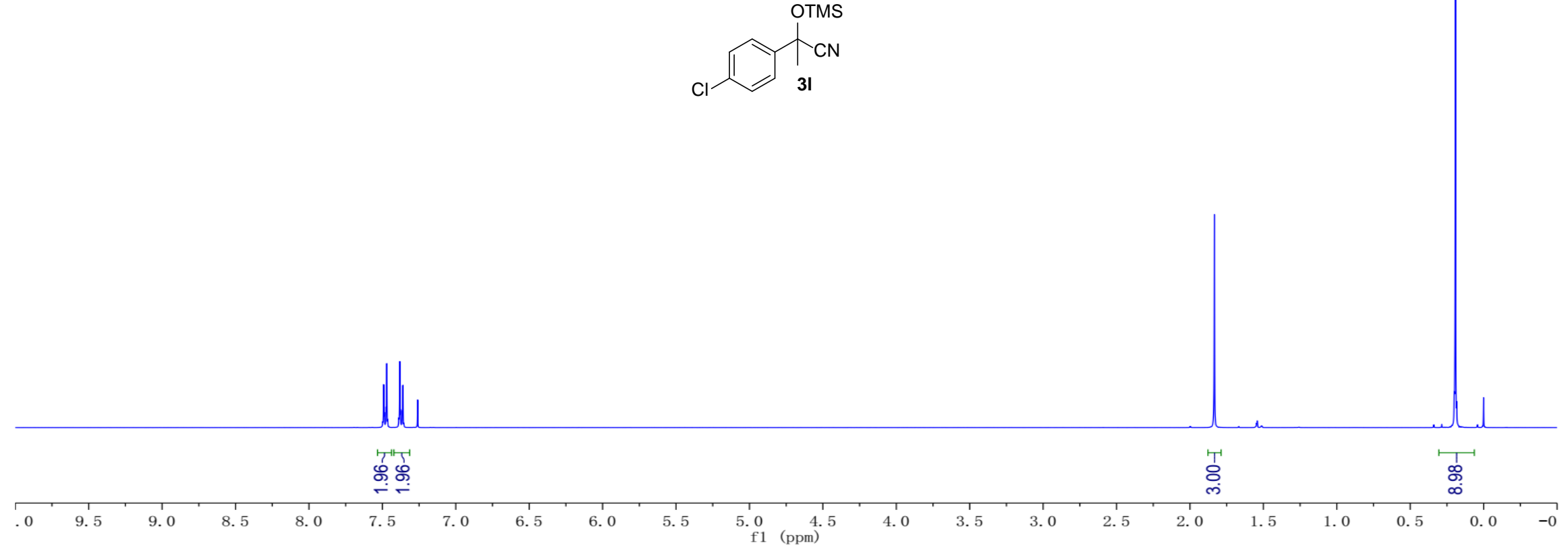
zxp-zm-133-3

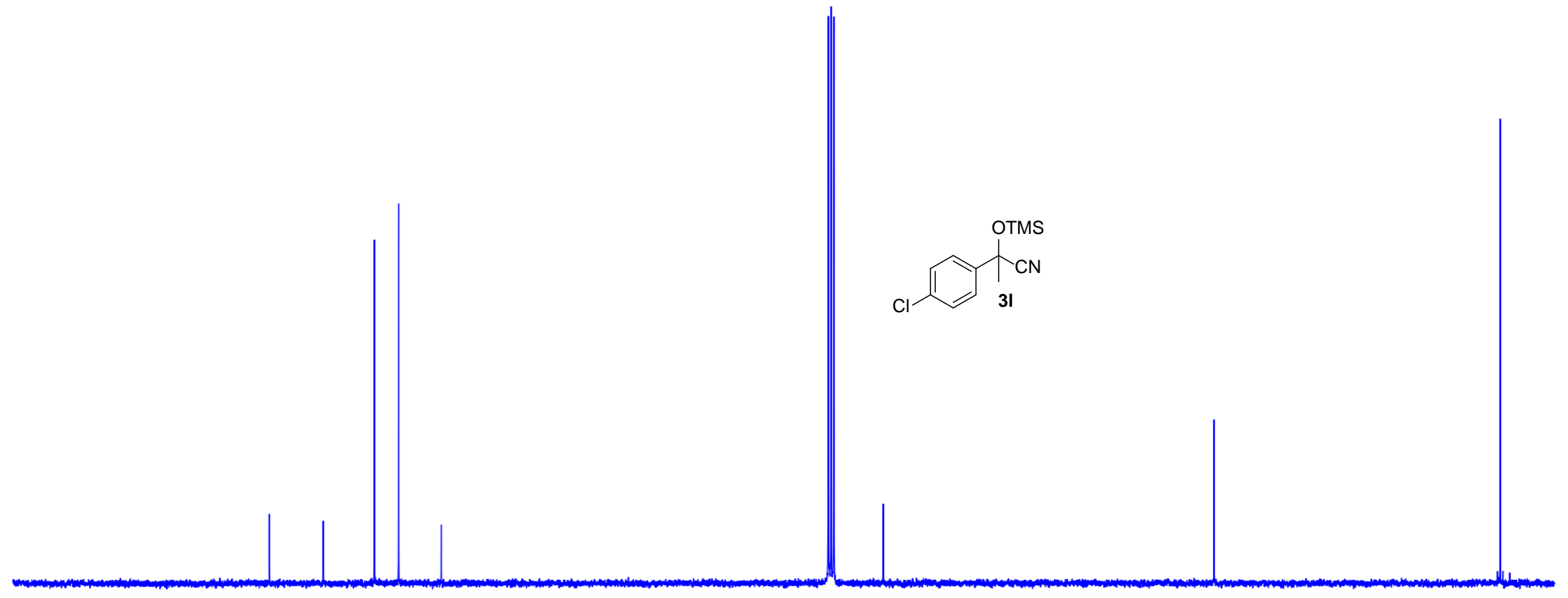

70 160 150 140 130 
Zxp-zm-138-1
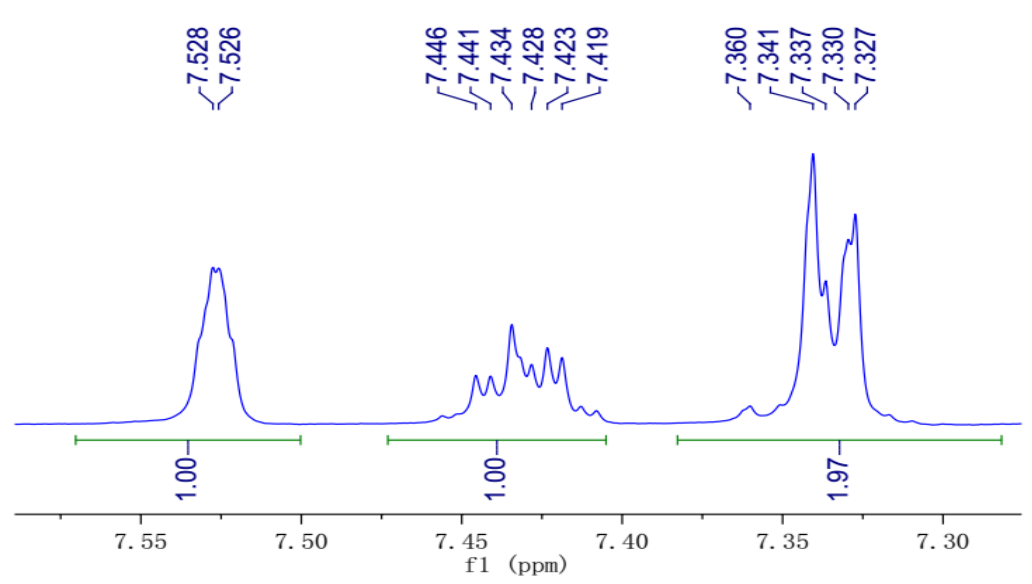

wil

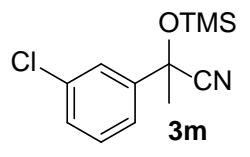

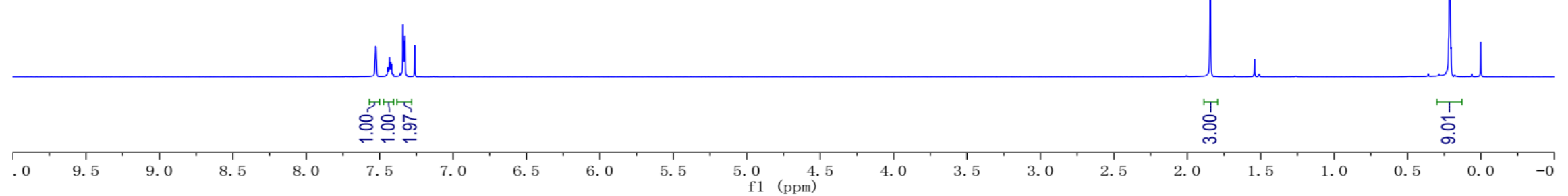


zxp-zm-138-1

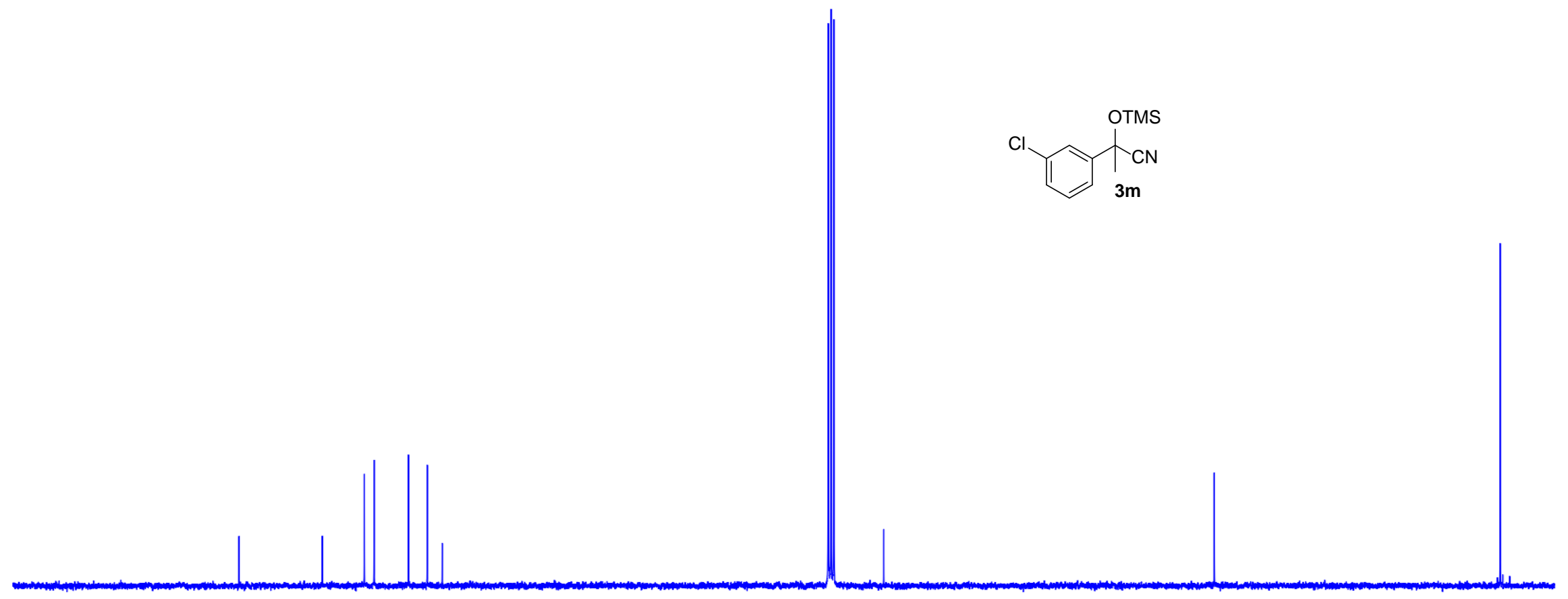

70 160 150 


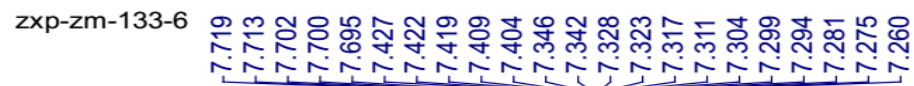

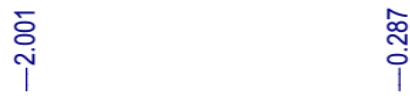
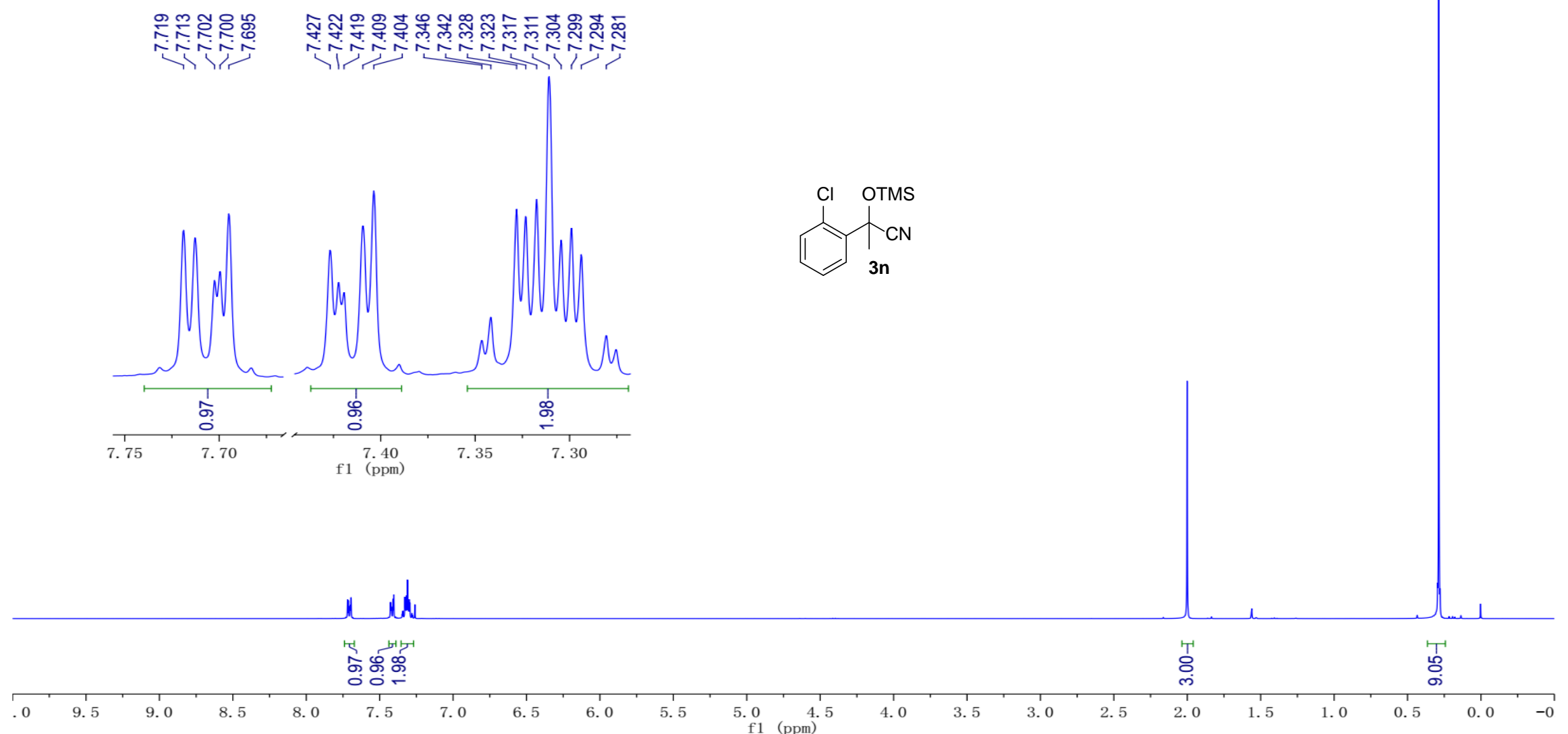
zxp-zm-133-6

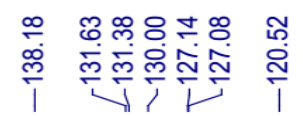

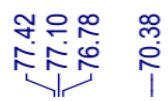

รั.

$\underbrace{C l}_{3 n}$

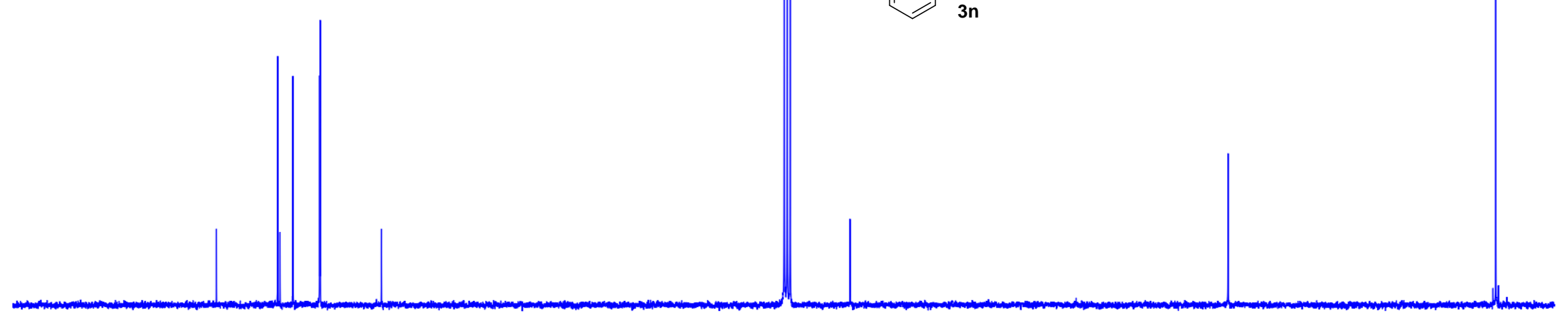

30

150

140

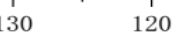

110

100

90

80

60

50

40

30

20

10 


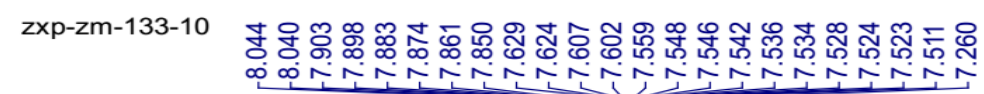

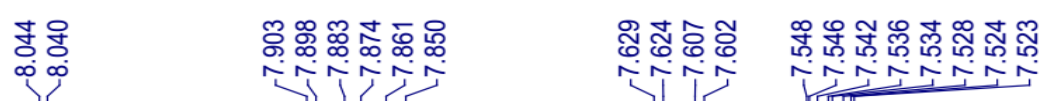
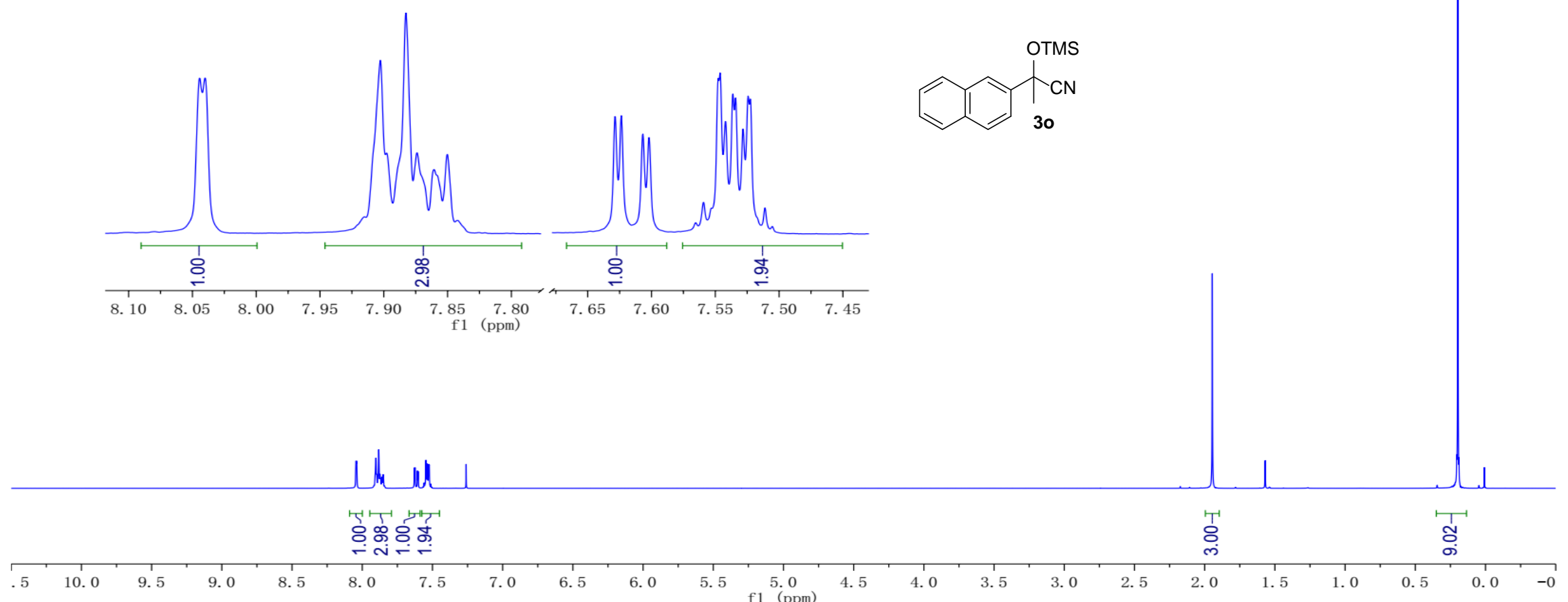


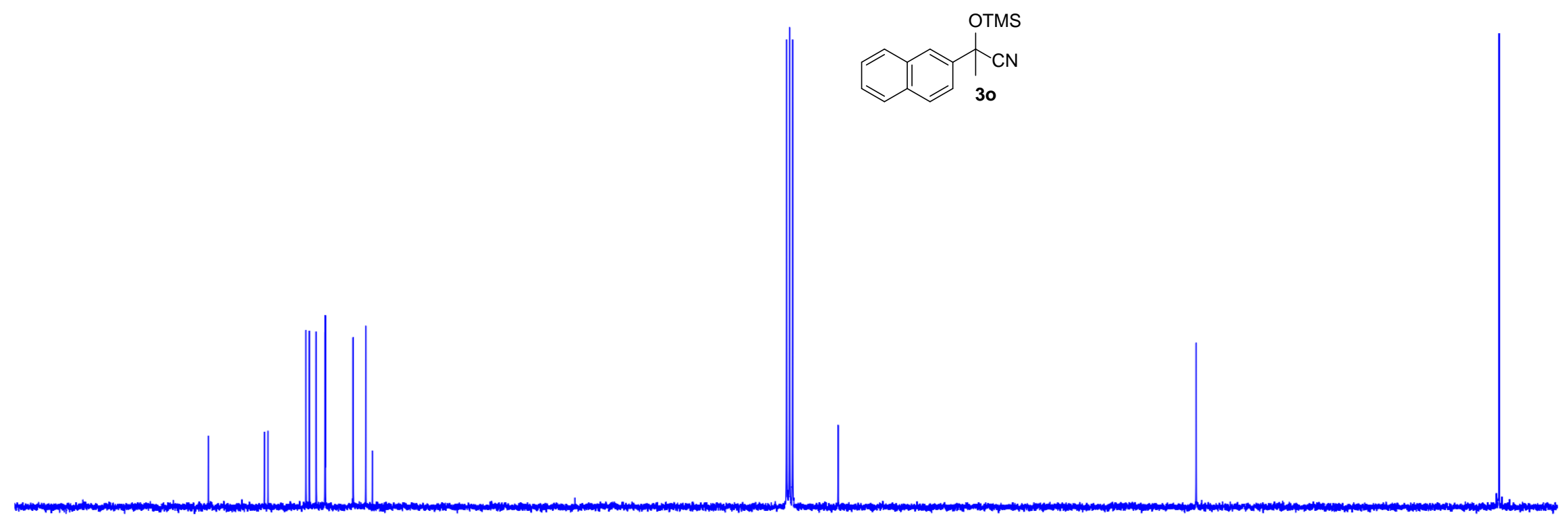

30 


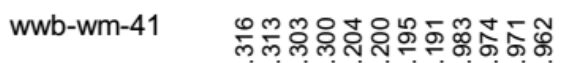

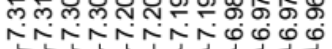

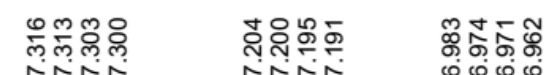

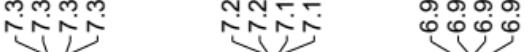

OTMS

¿ITCN

3p

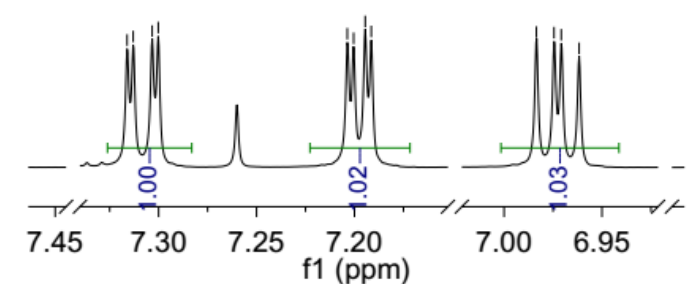

ili

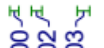

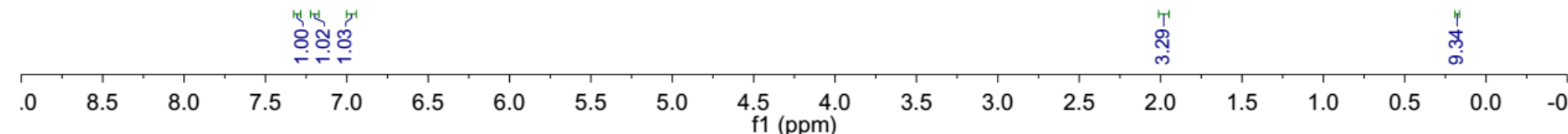


wwb-wm-41

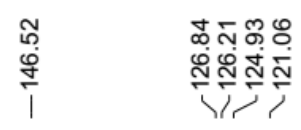

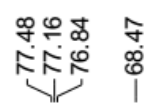

অ্ল

$\stackrel{\text { กั }}{\leftarrow}$

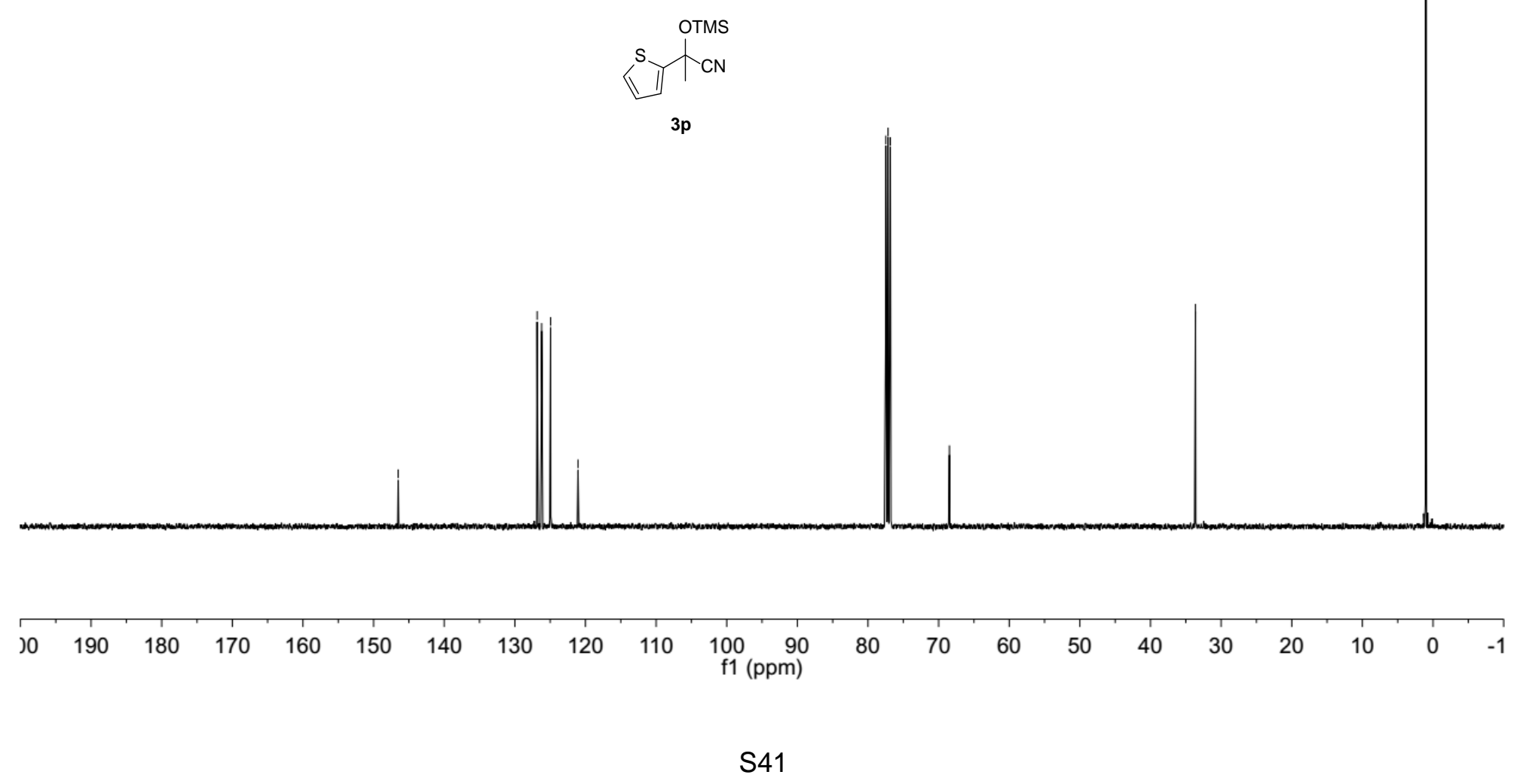




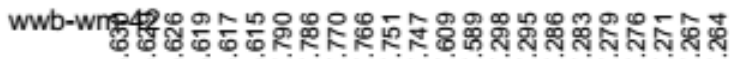

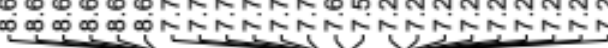
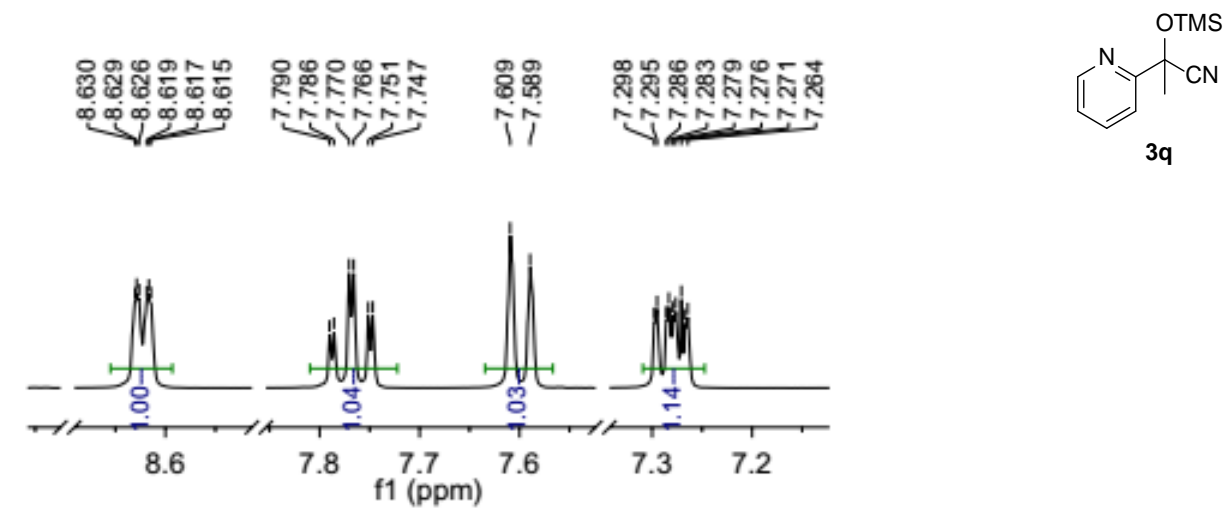

39

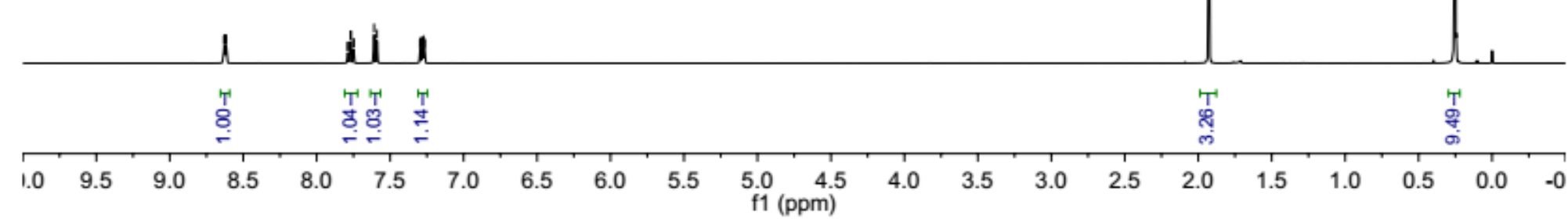


wwb-wm-42

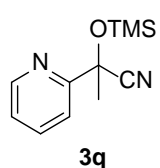

$3 q$

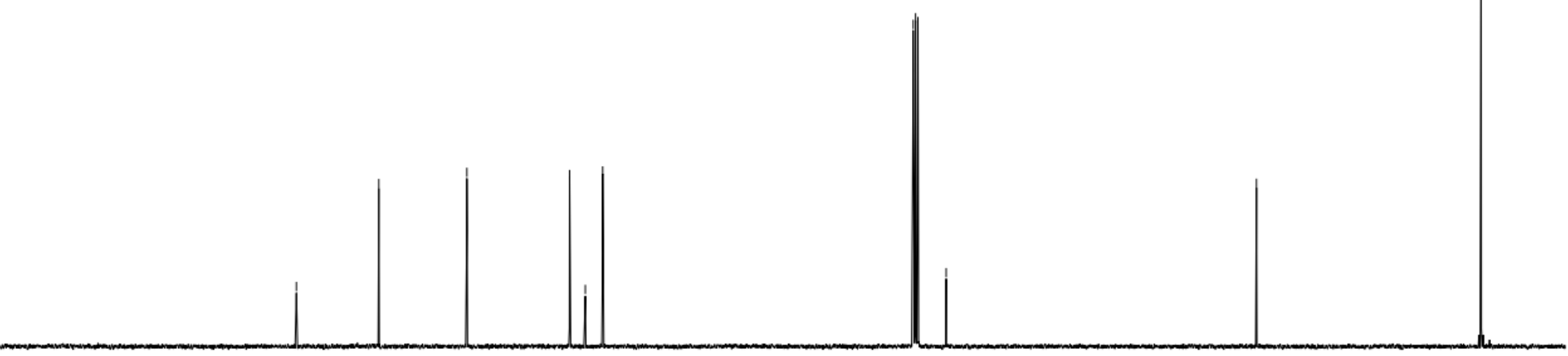

$\begin{array}{llllllllllll}0 & 190 & 180 & 170 & 160 & 150 & 140 & 130 & 120 & 110 & 100 & 90\end{array}$ f1 (ppm) 


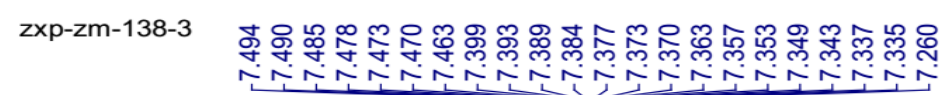

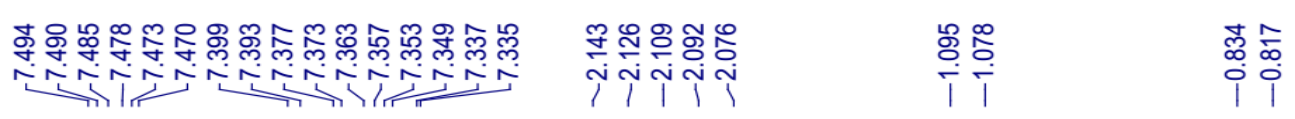
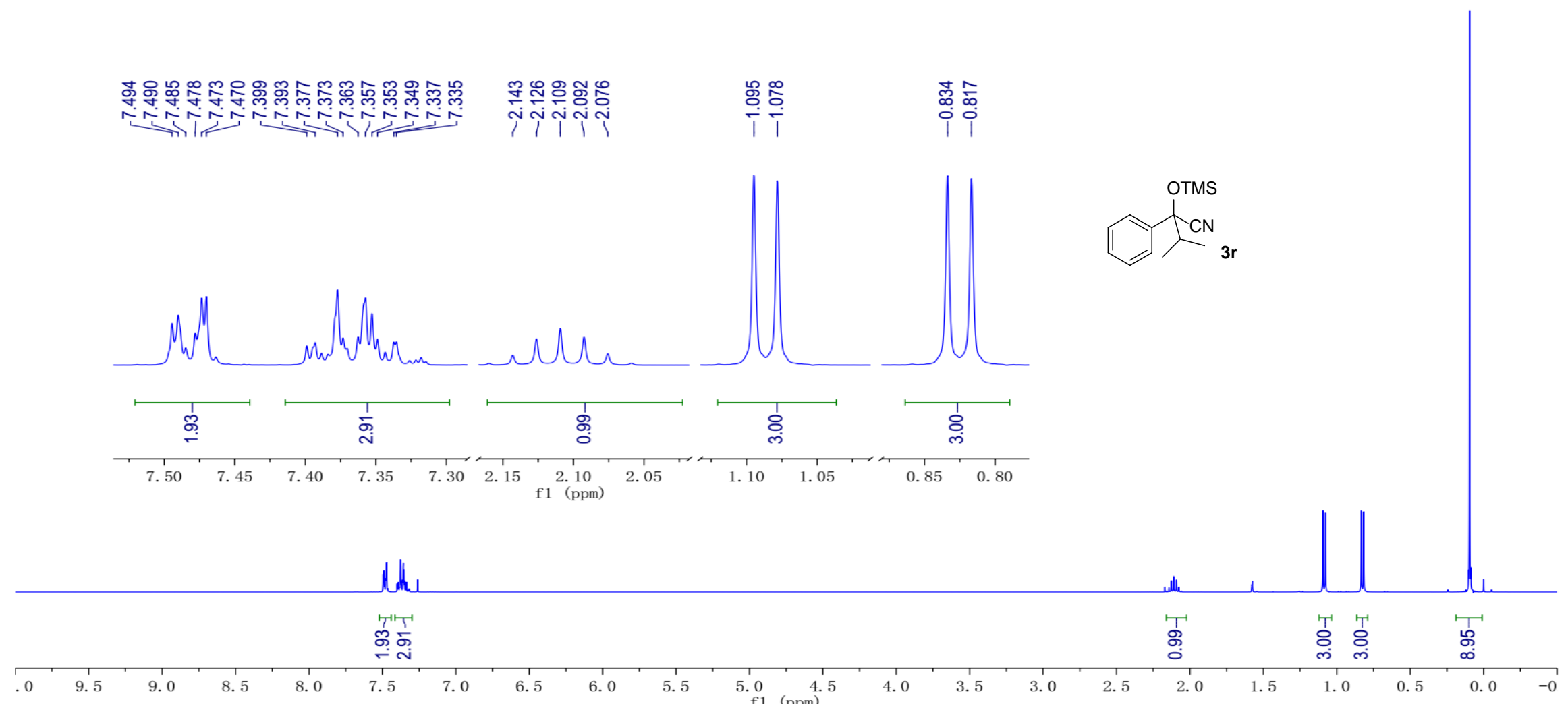
zxp-zm-138-3

욤

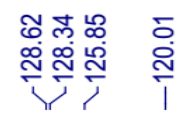

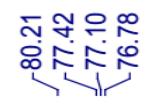

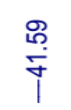

产

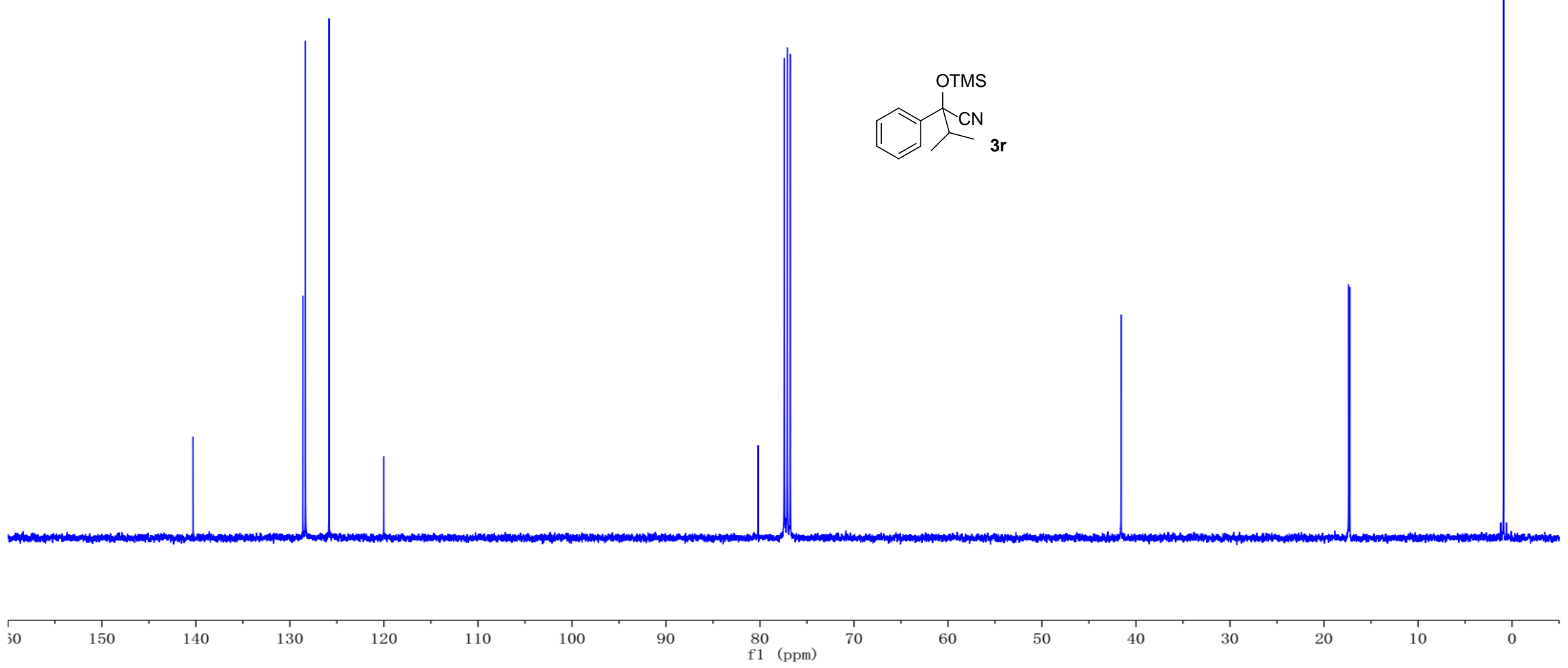

S45 
zxp-zm-133-11

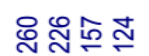

लुलिल

8
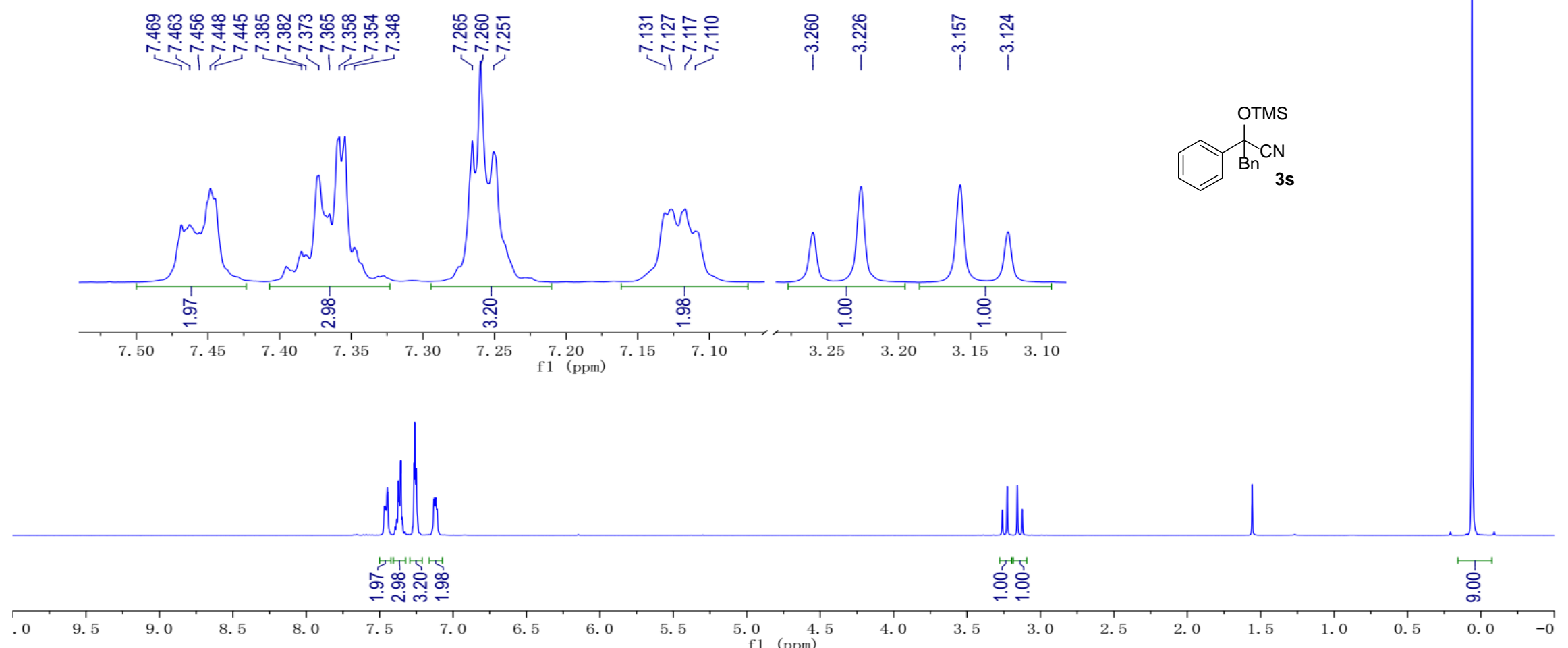
zxp-zm-133-11

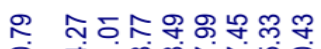

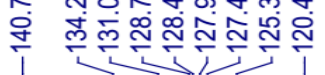

군은요용

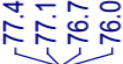

ָิ

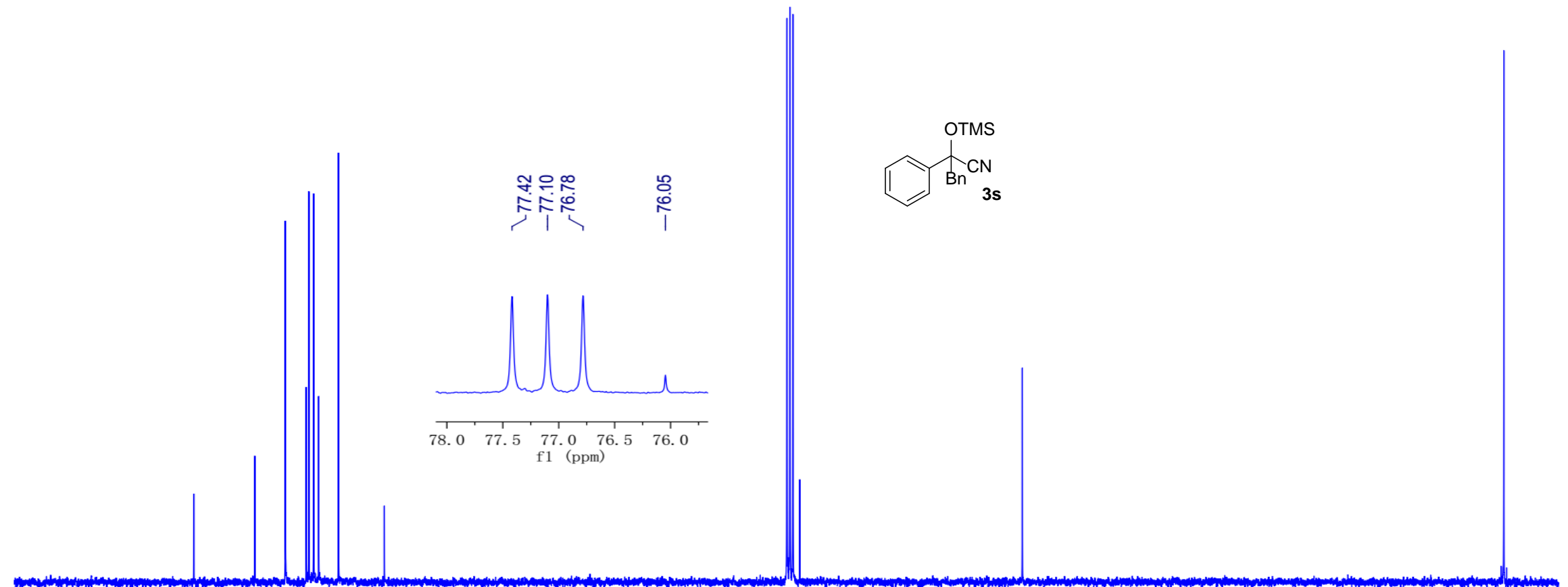

io 150 140
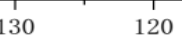

110

100

90

80
$\mathrm{f} 1$$(\mathrm{ppm})$

60

50

40

30

$20 \quad 10$ 


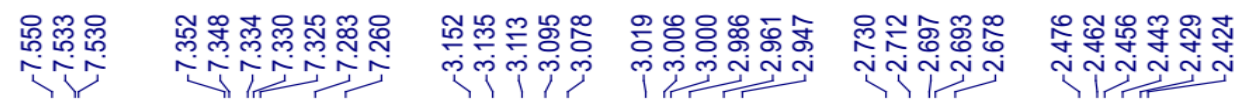
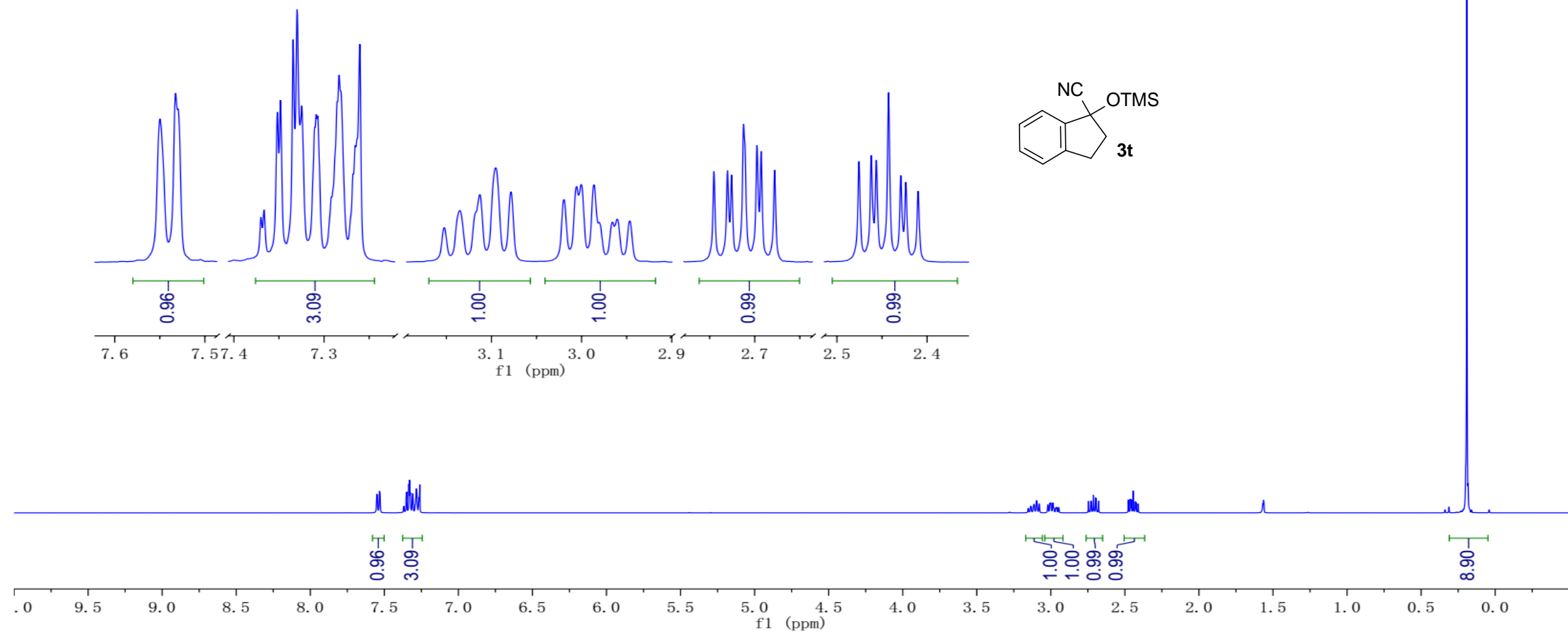


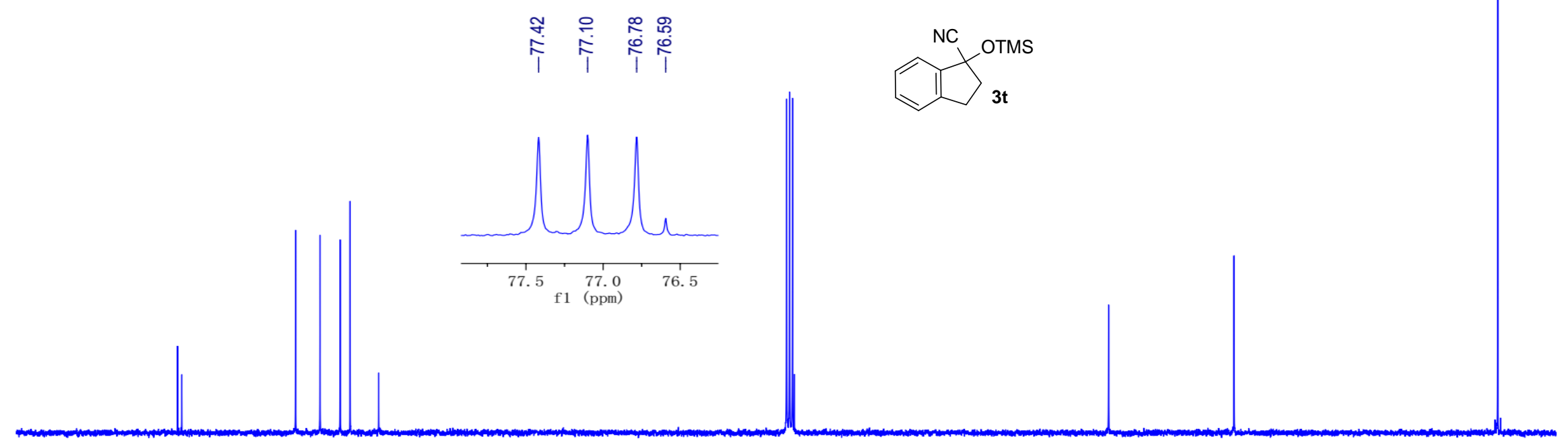

30 150 


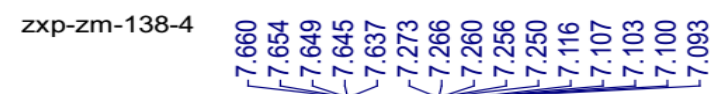

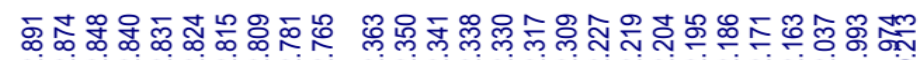
نN

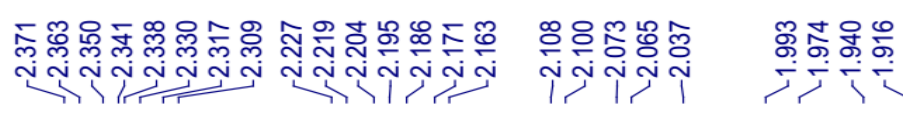
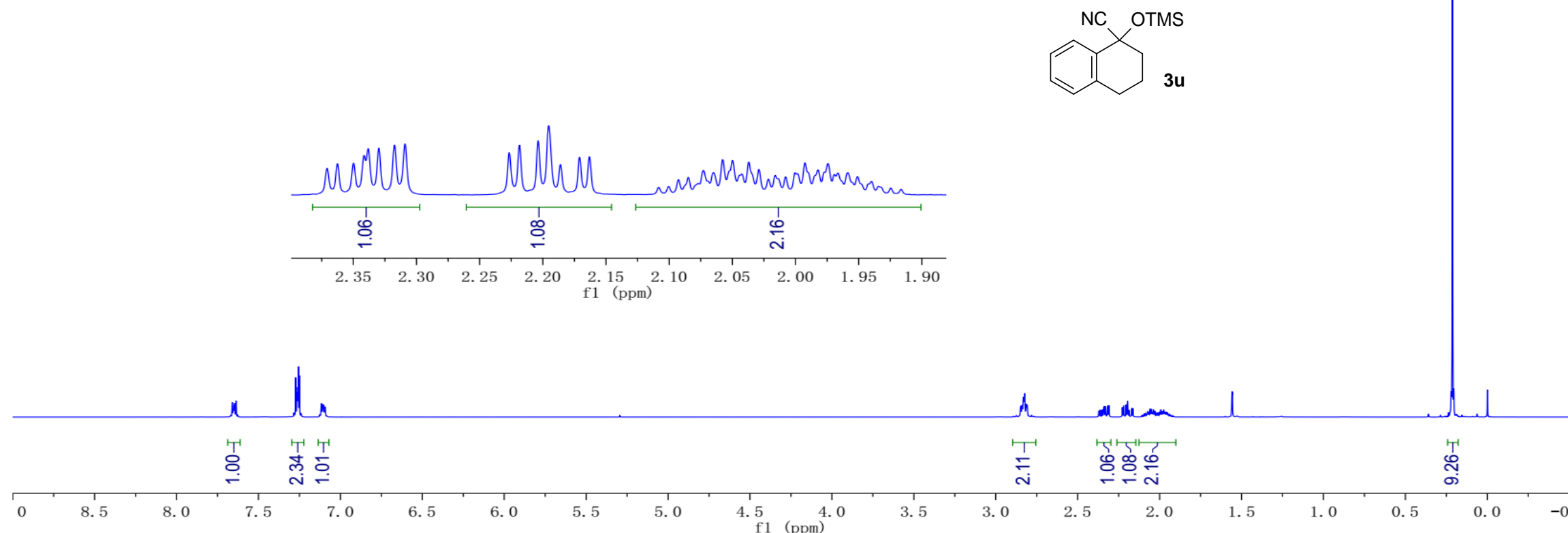

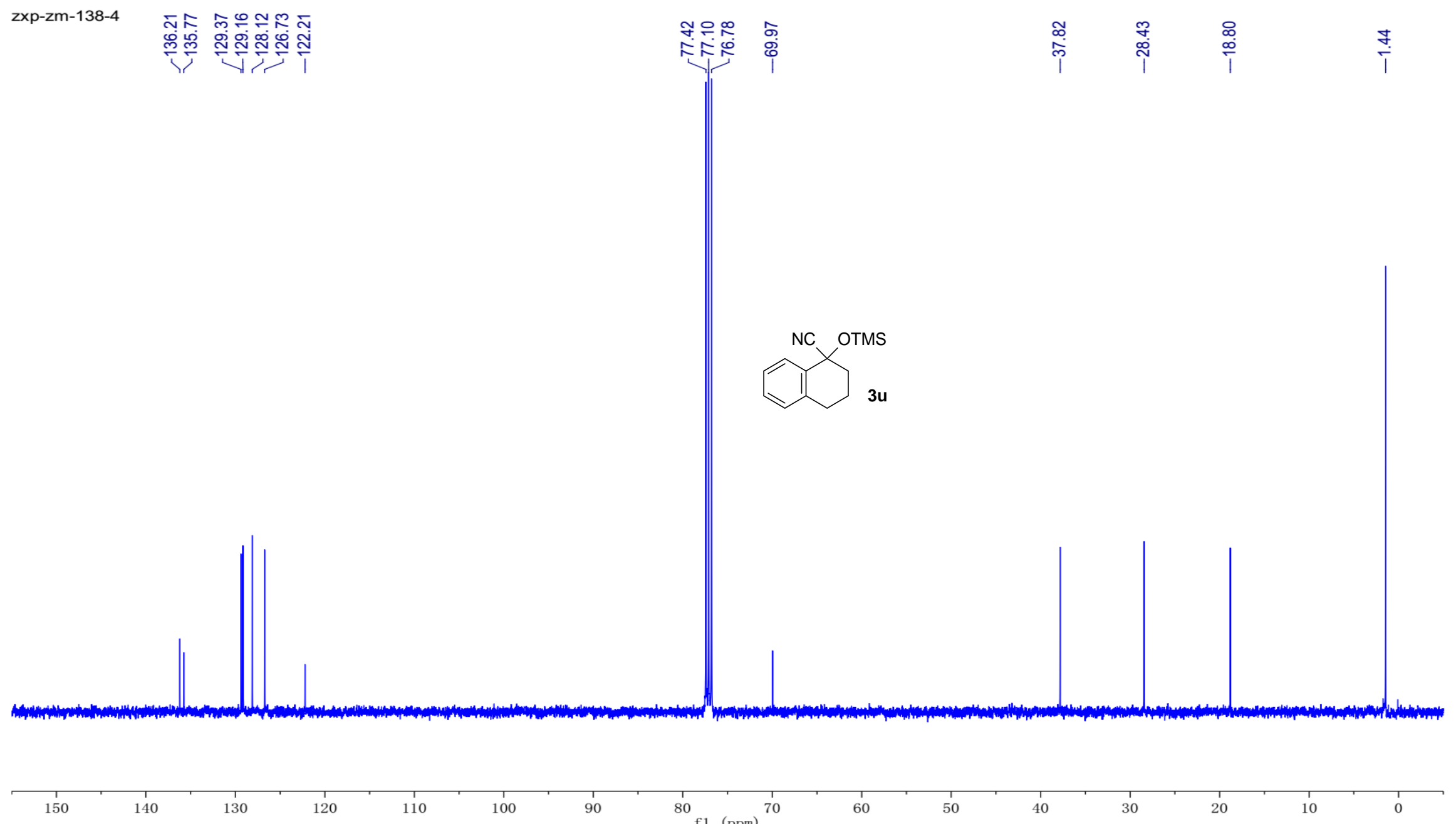
zxp-zm-140-1

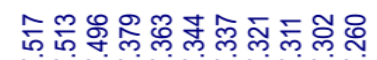

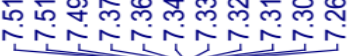

OTMS

$\triangle \mathrm{Ph}_{3 v}^{\mathrm{CN}}$

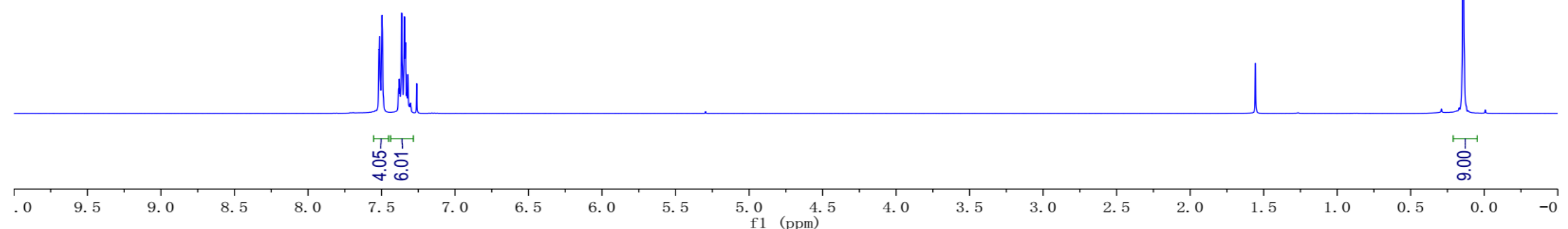




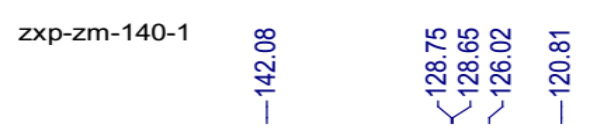

굿유웡

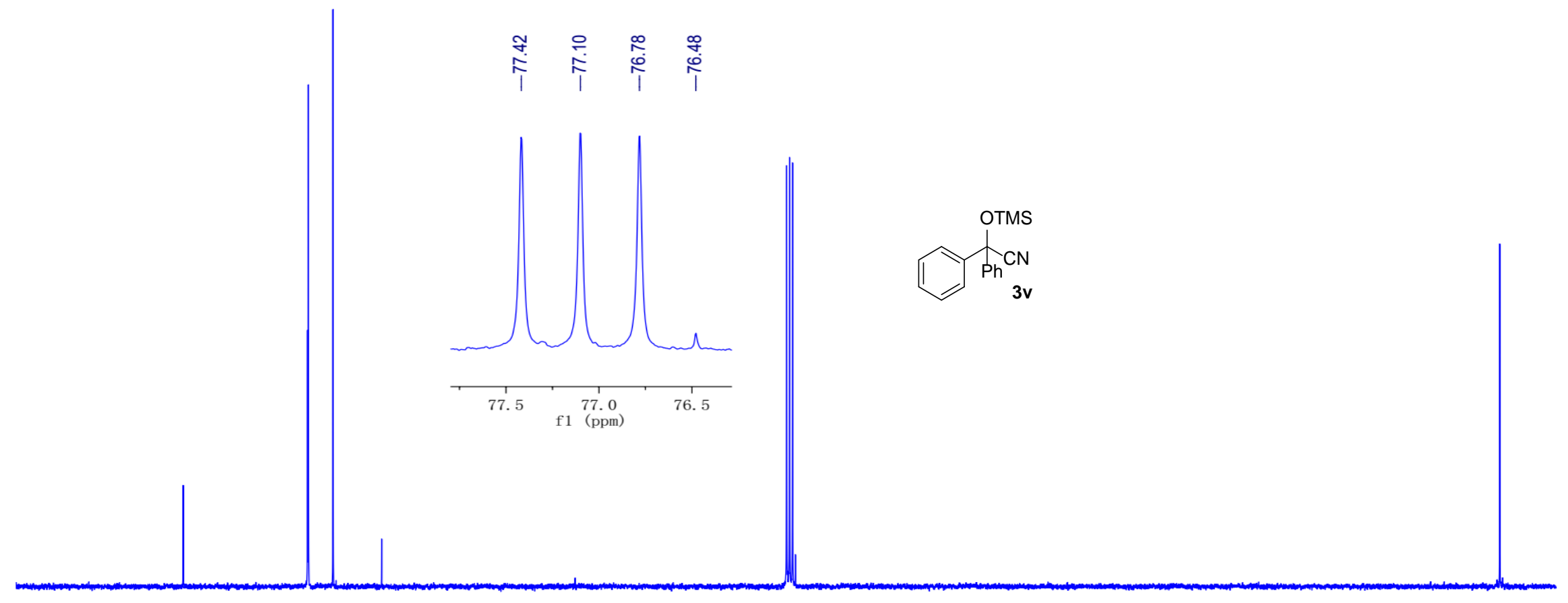

30 150 140 $130 \div \quad 120$ $110 \quad 100$ 90 80 60 50 40 30 $20+10$ 0 


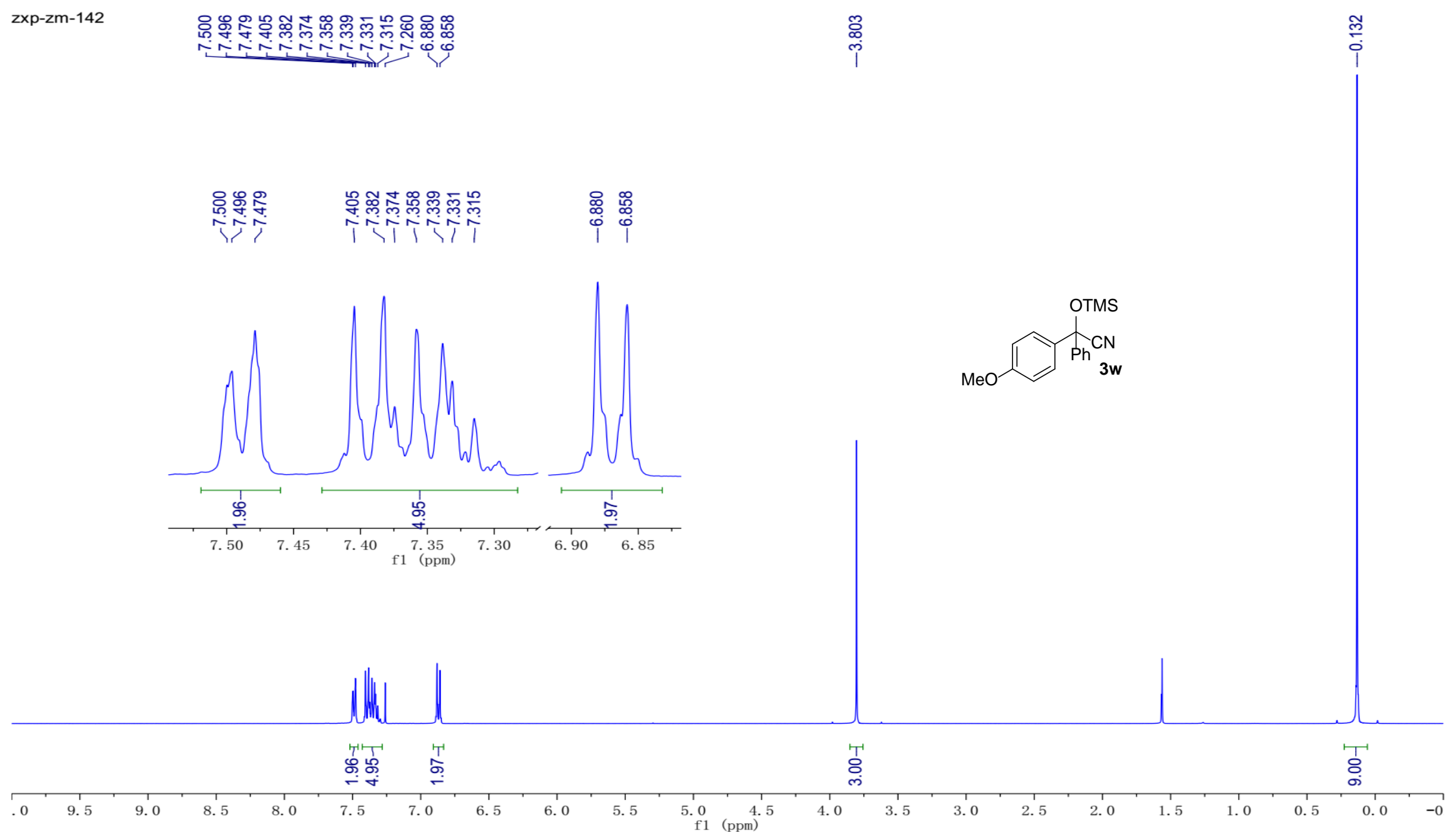


zxp-zm-142-p 商
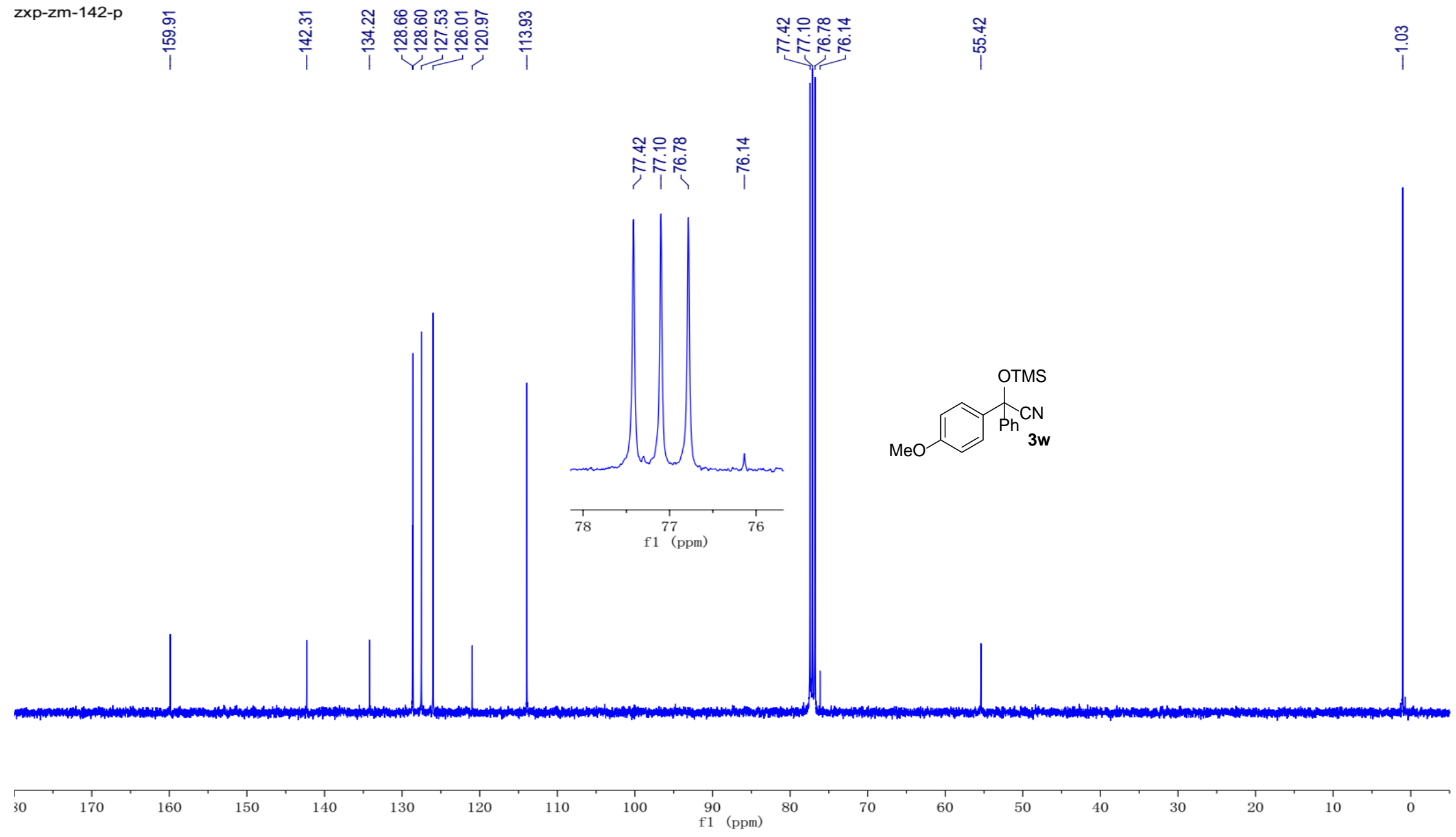

买

$\stackrel{\infty}{i}$

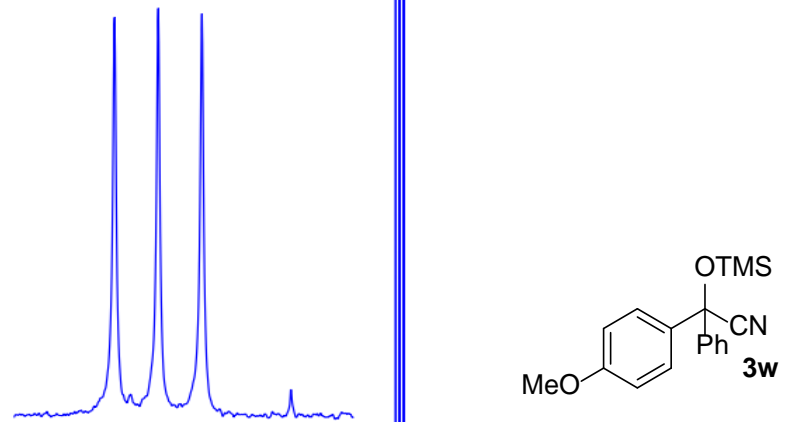

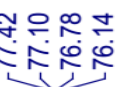

S55 
2xp-zm-141-2

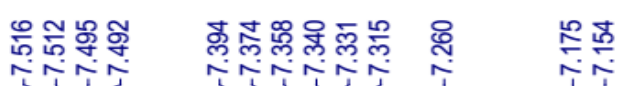
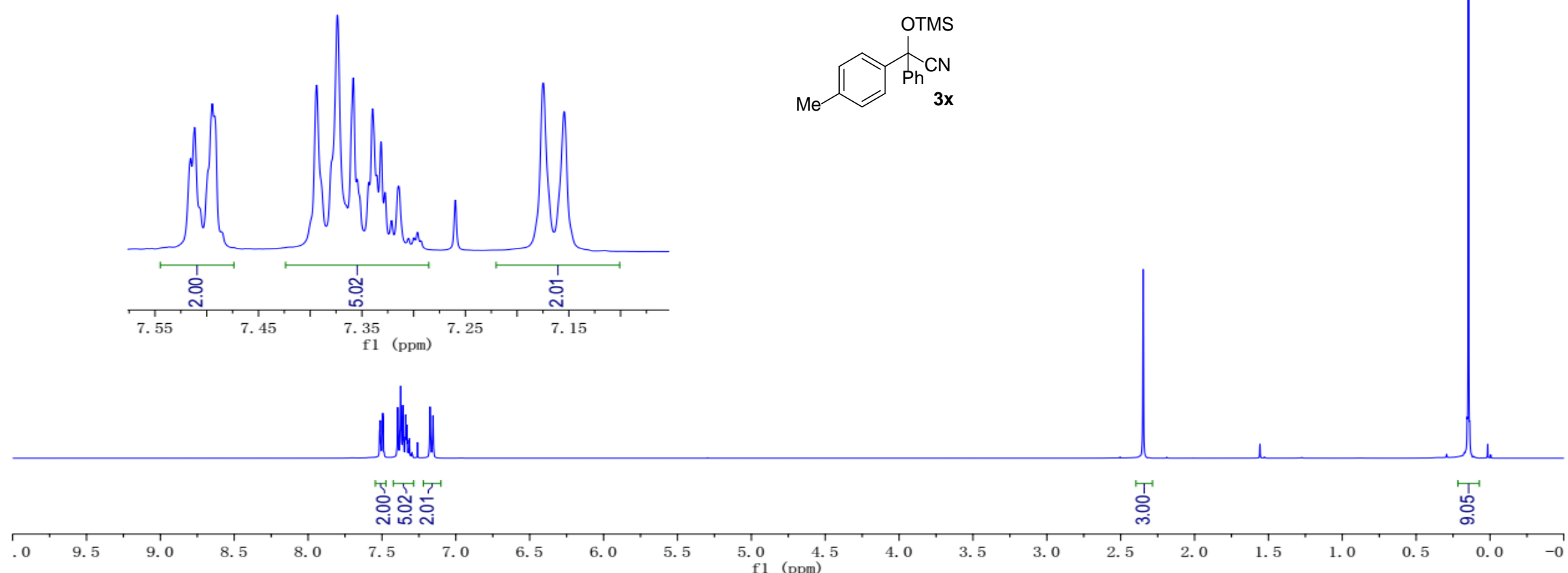

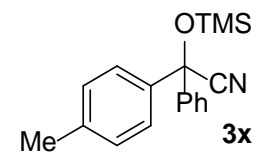



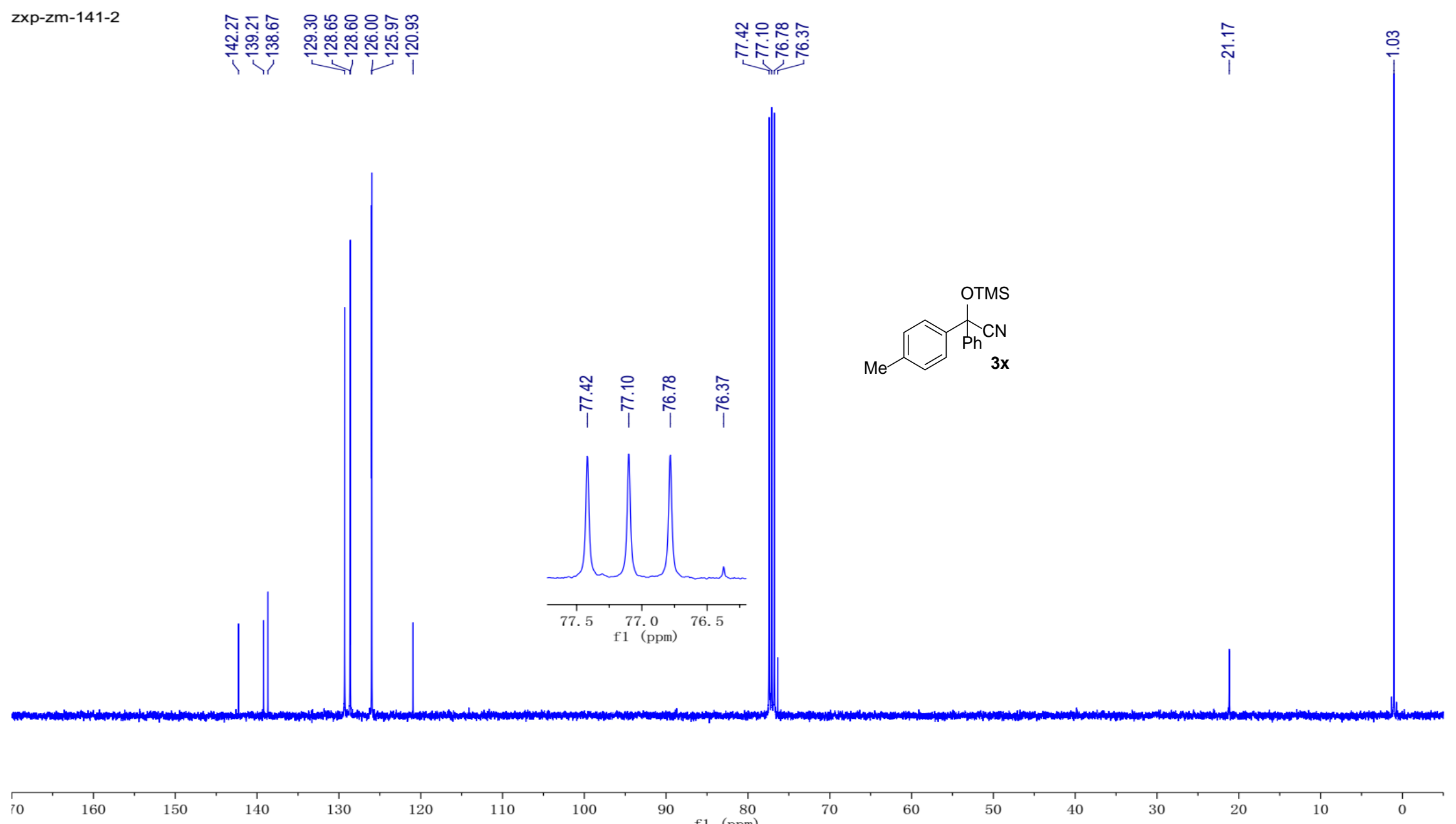


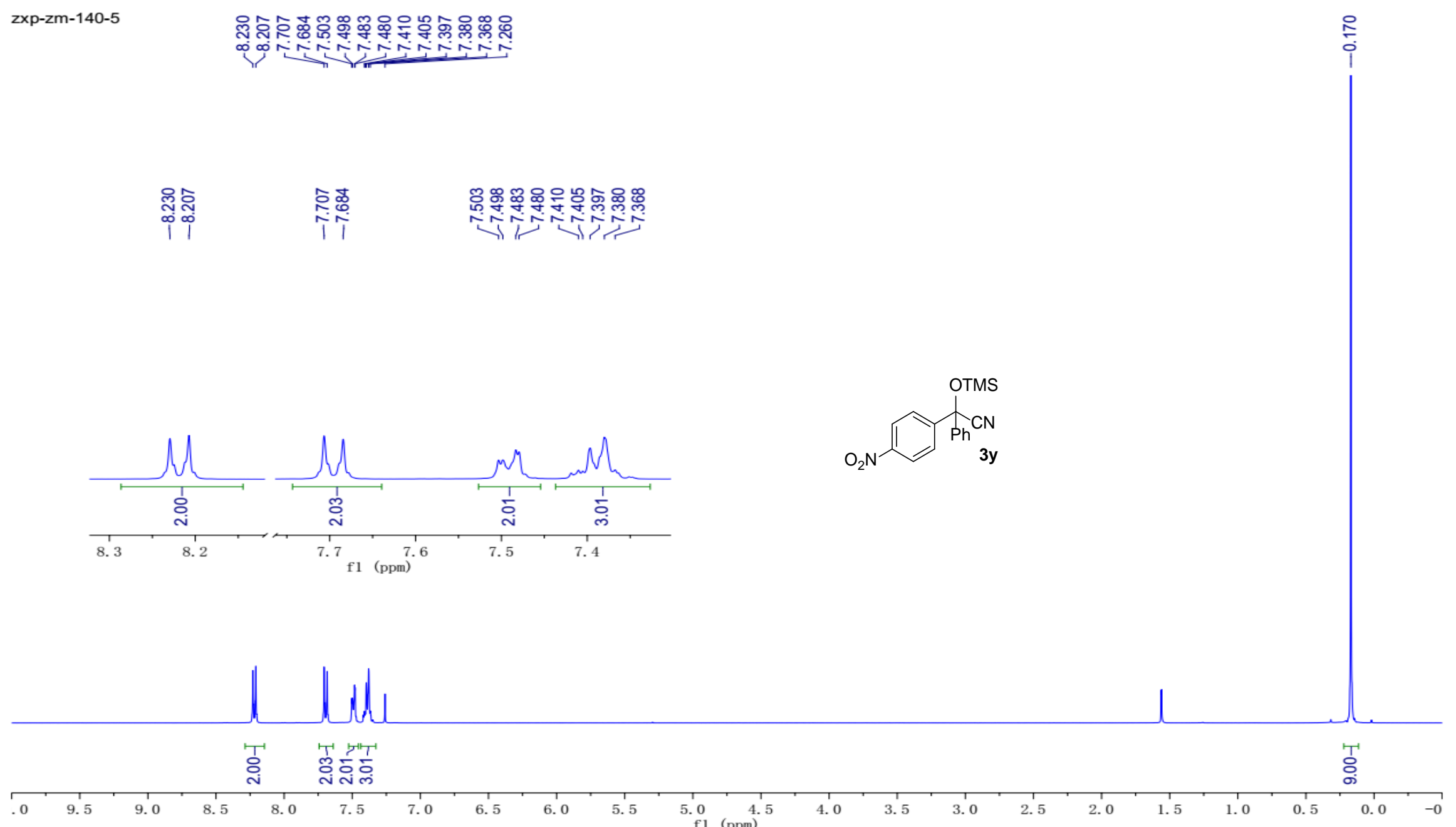




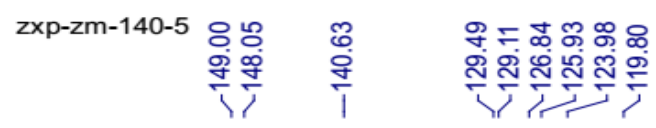
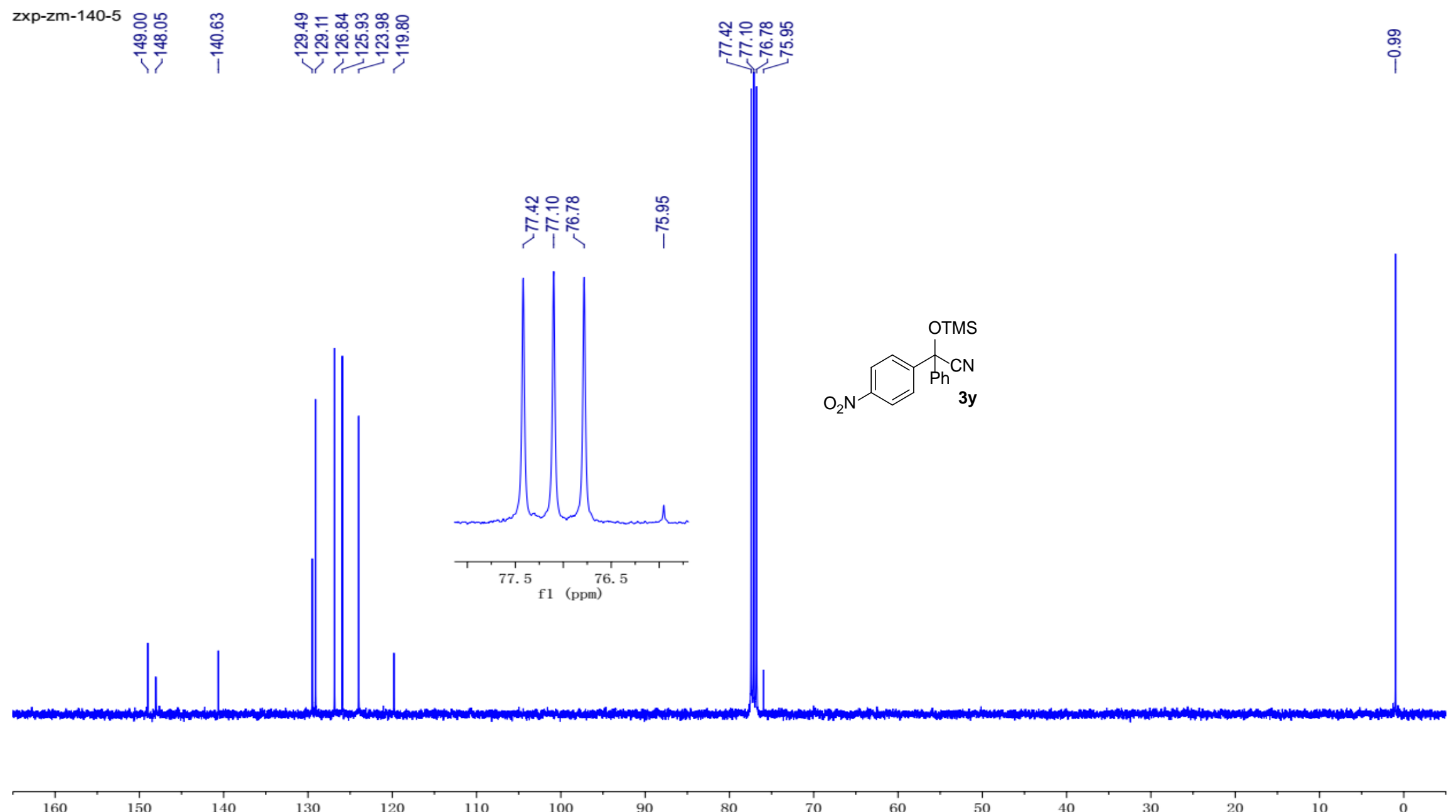

140

130

120

110

100

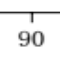

${ }^{1} 0$

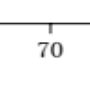

60

50

40

30

20 
zxp-zm-141-4

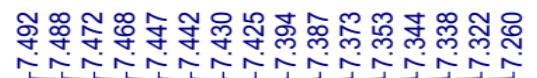

웅

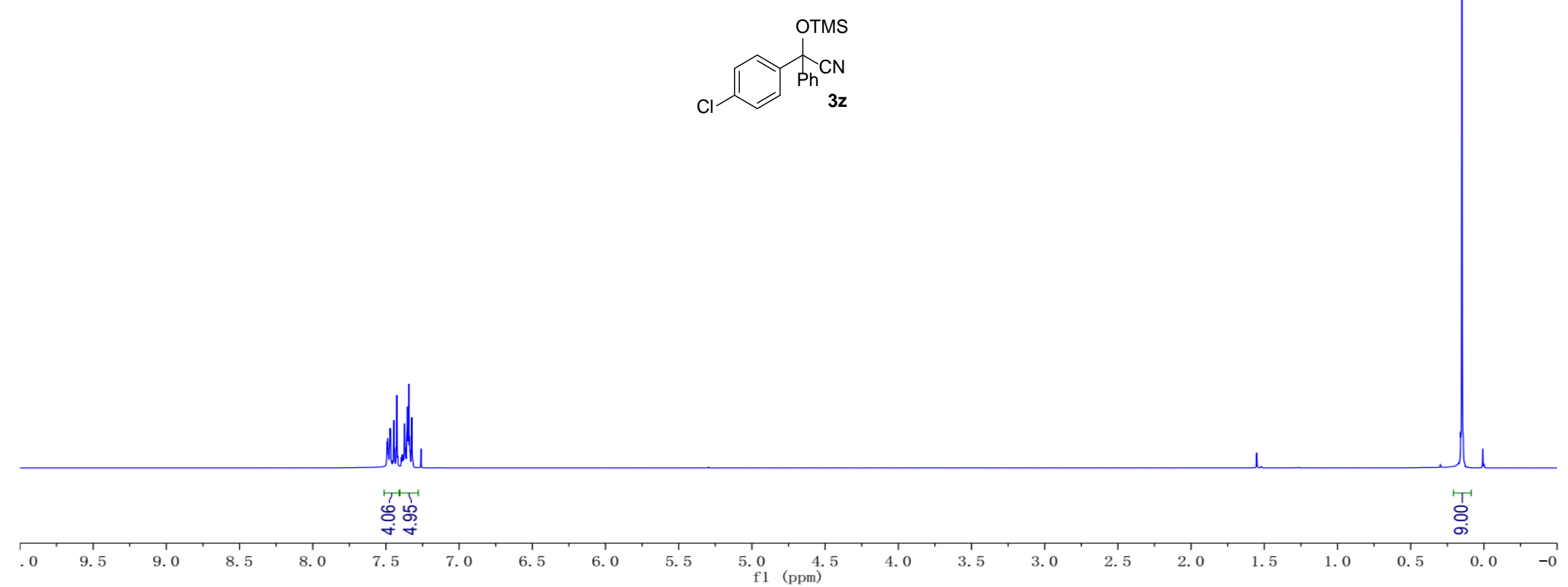




$$
I L
$$


zxp-zm-141-1

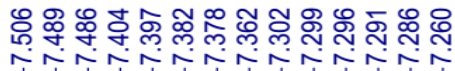
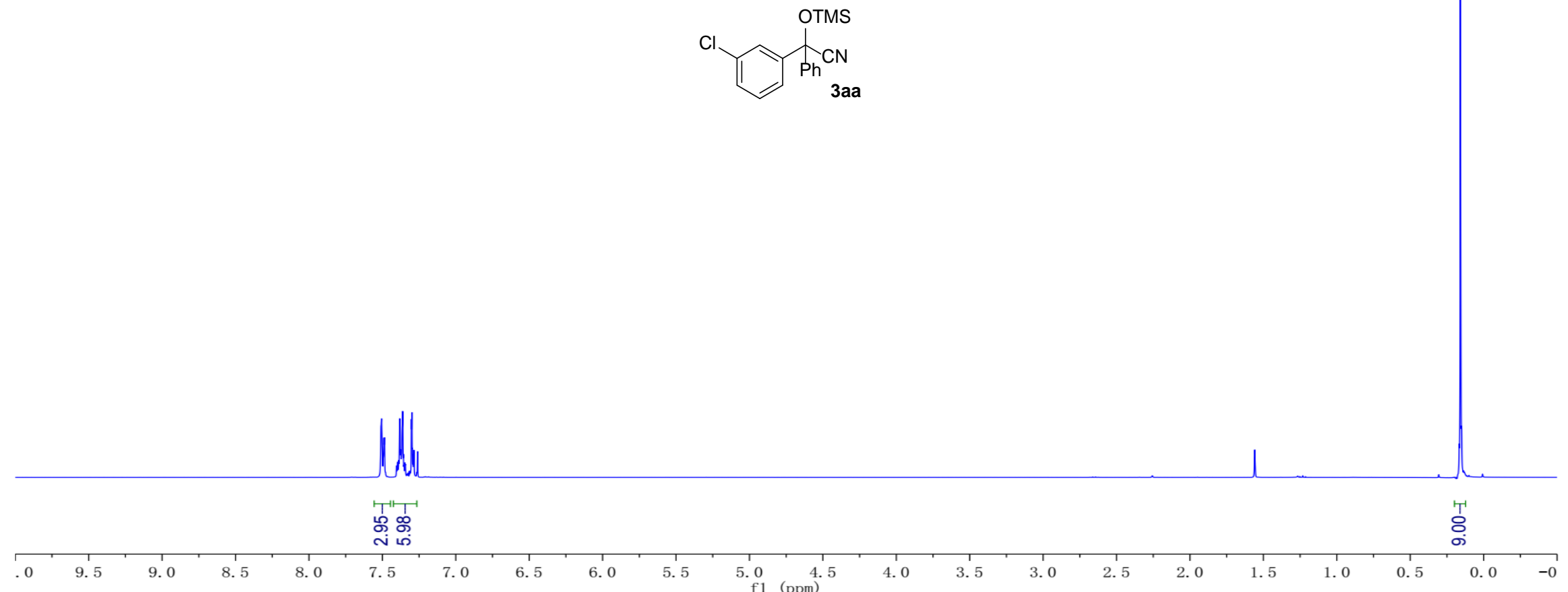
zxp-zm-141-1

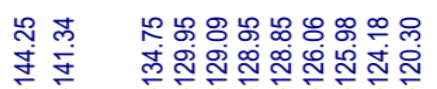

웅요

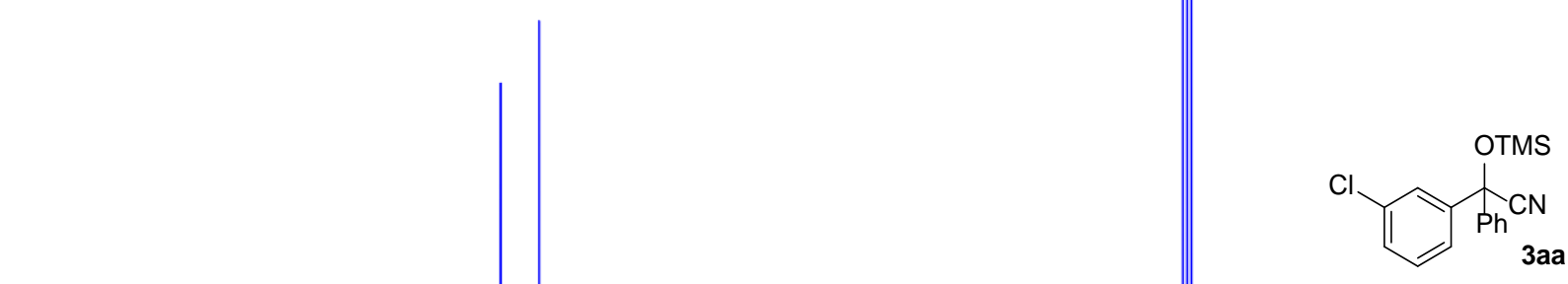




$$
\overbrace{3 a b}^{\mathrm{Cl}}
$$

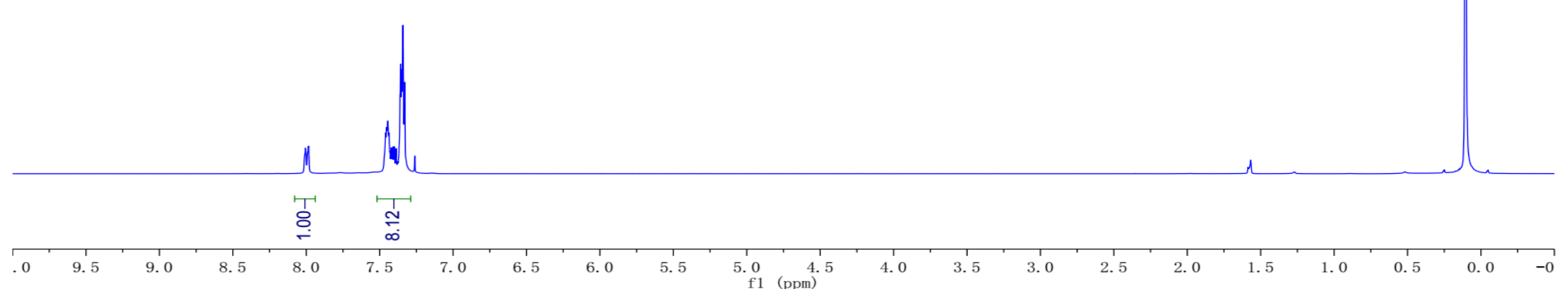




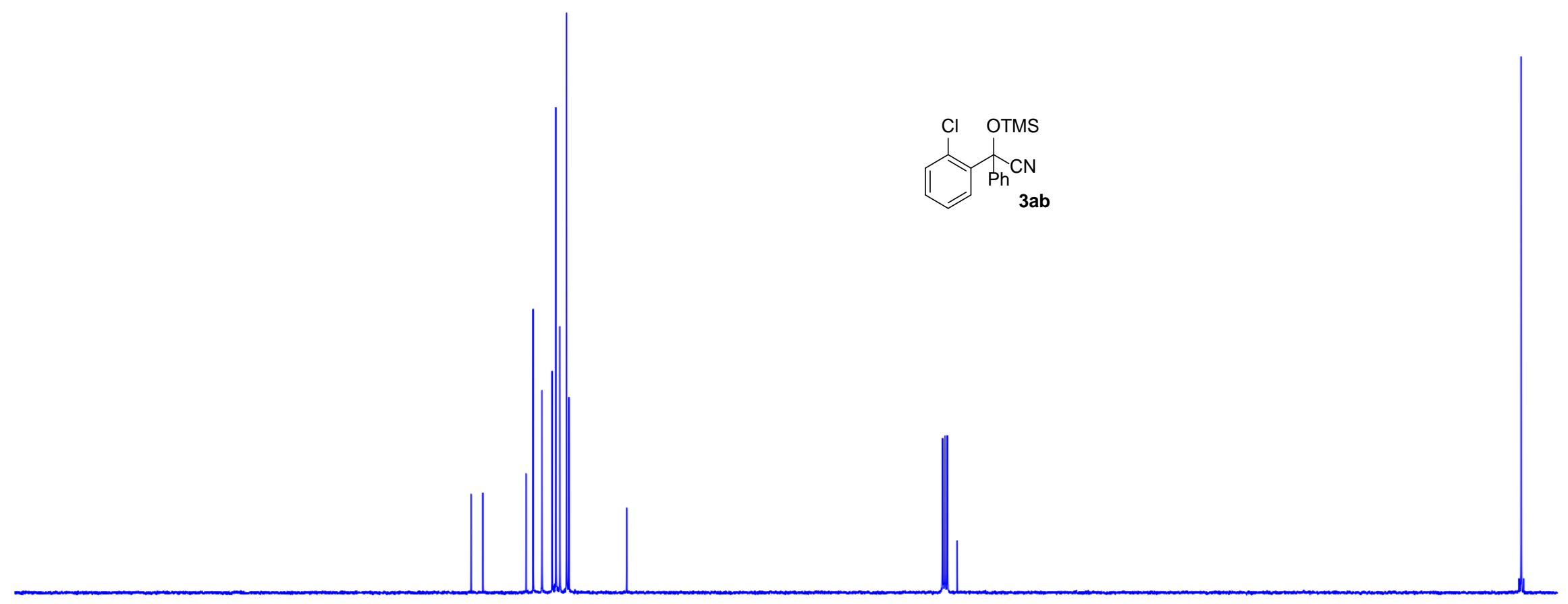

$10 \times 190,180,170,160,150,140,130$

$120 \quad 110$

$100 \quad 90$

80

$70 \quad 60$

$50 \quad 40$

$30+20 \cdot 10$

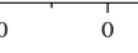




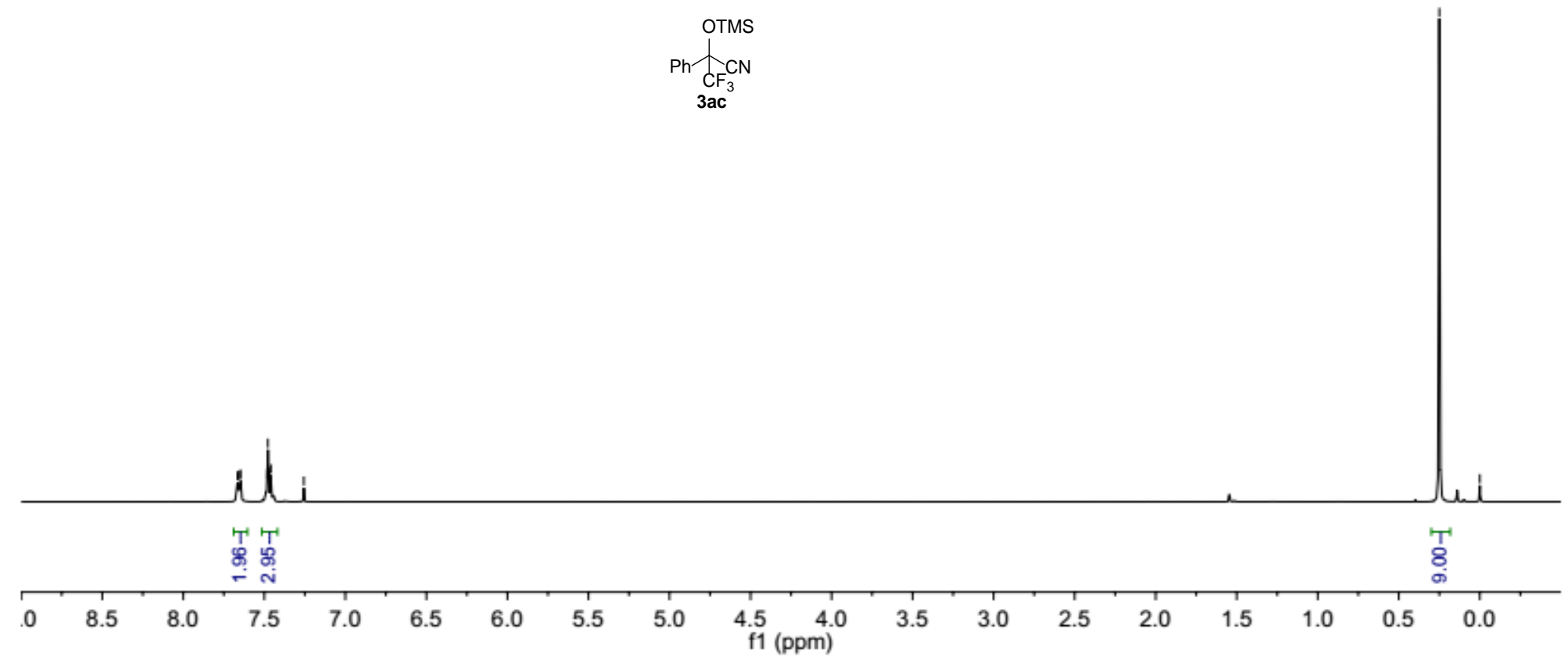




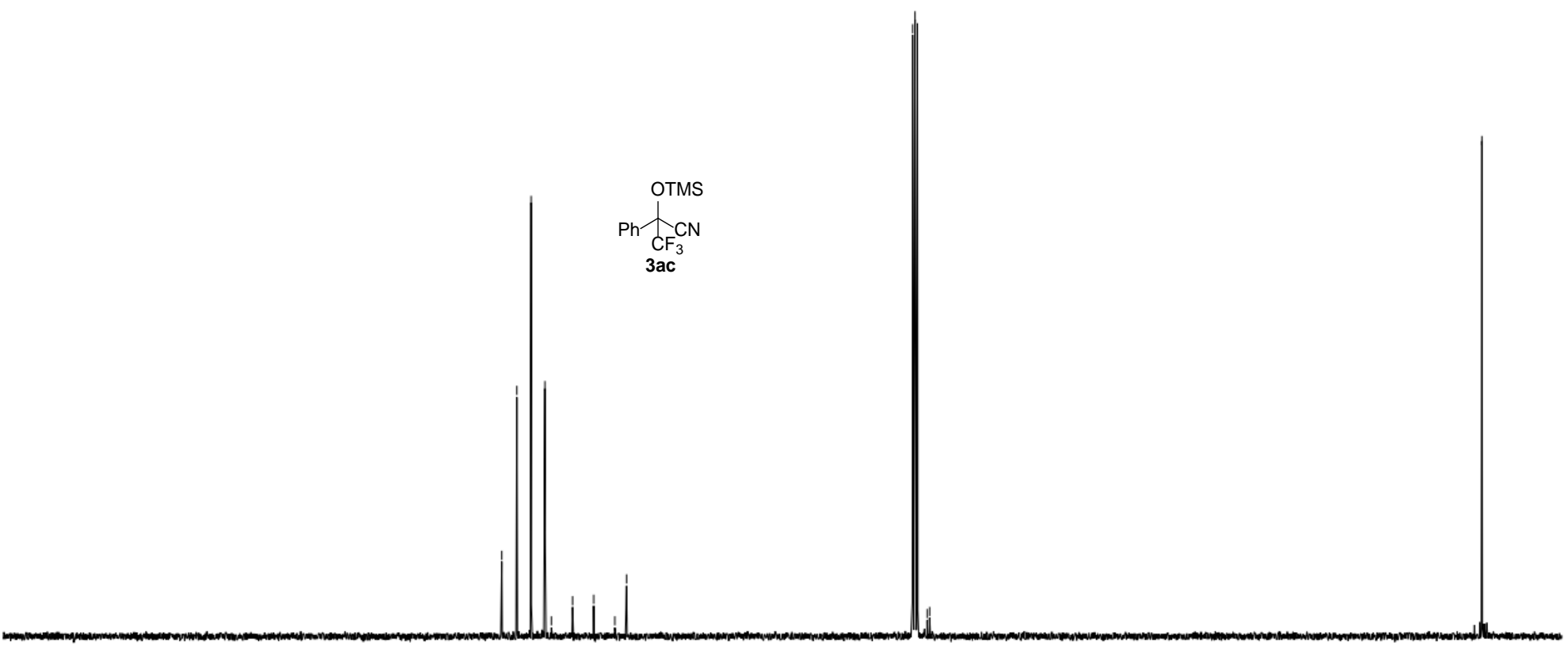

$\begin{array}{lllllllllll}190 & 180 & 170 & 160 & 150 & 140 & 130 & 120 & 110 & 100 & 90 \\ & & & & & & & & \end{array}$ 
wwb-wk-121

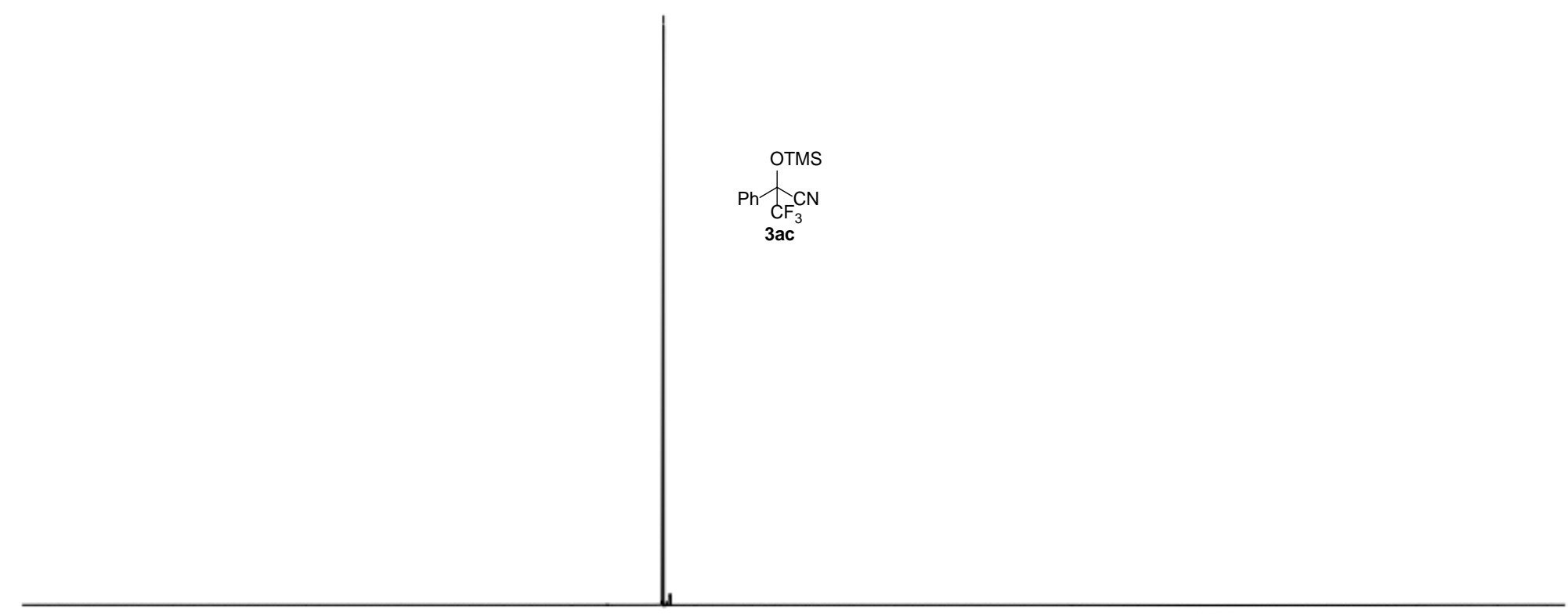

$\begin{array}{llllllllllllllllllllllllllllllllllll}10 & 0 & -10 & -20 & -30 & -40 & -50 & -60 & -70 & -80 & -90 & -100 & -110 & -120 & -130 & -140 & -150 & -160 & -170 & -180 & -190 & -200 & -210\end{array}$ 


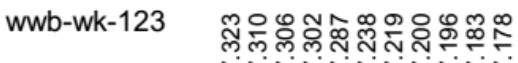

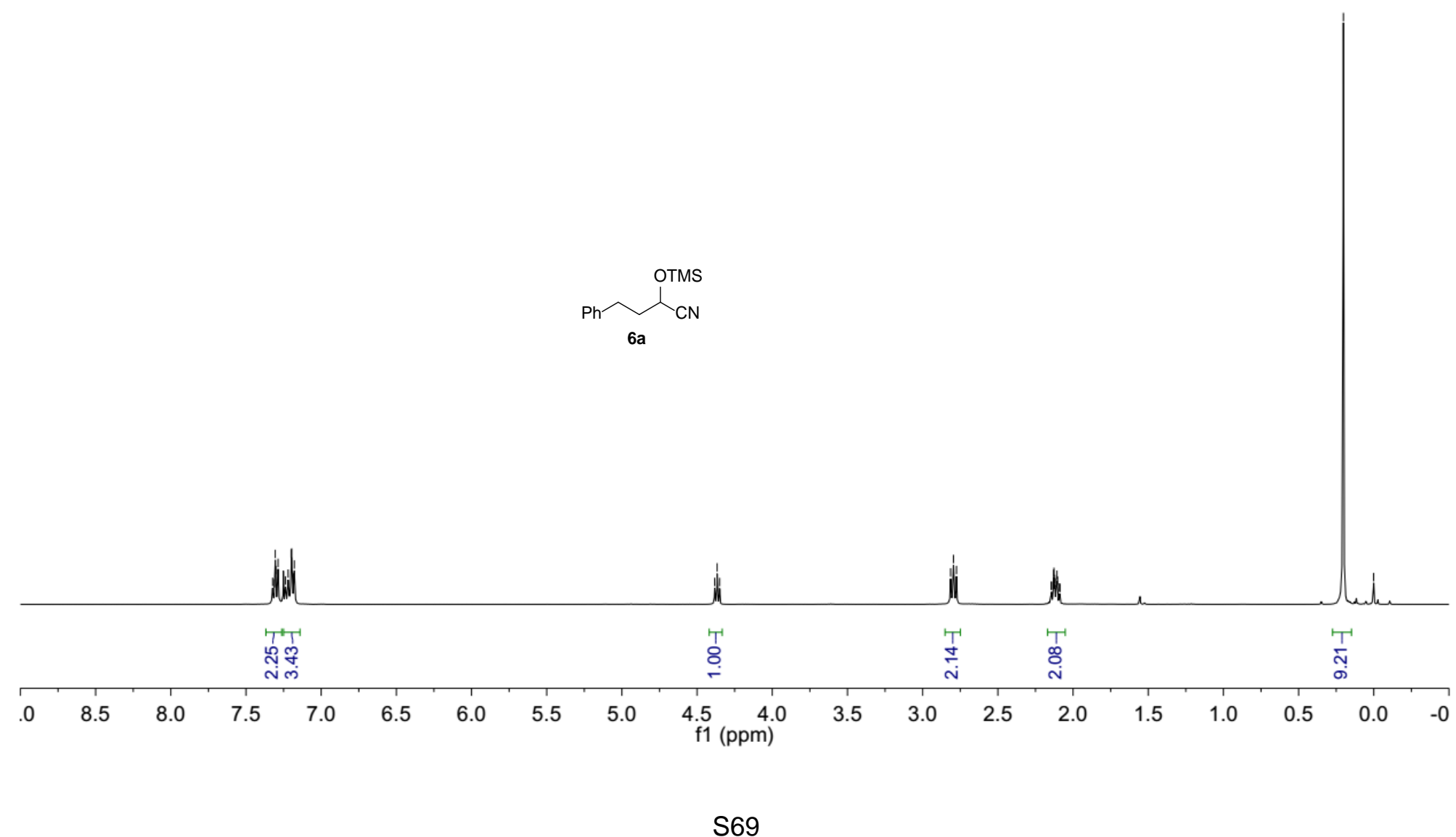


wwb-wk-123

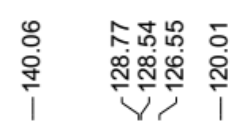

신

ळ.

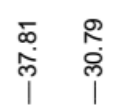

$\underset{\substack{\mathfrak{i} \\ \mathfrak{i}}}{\mathfrak{n}}$
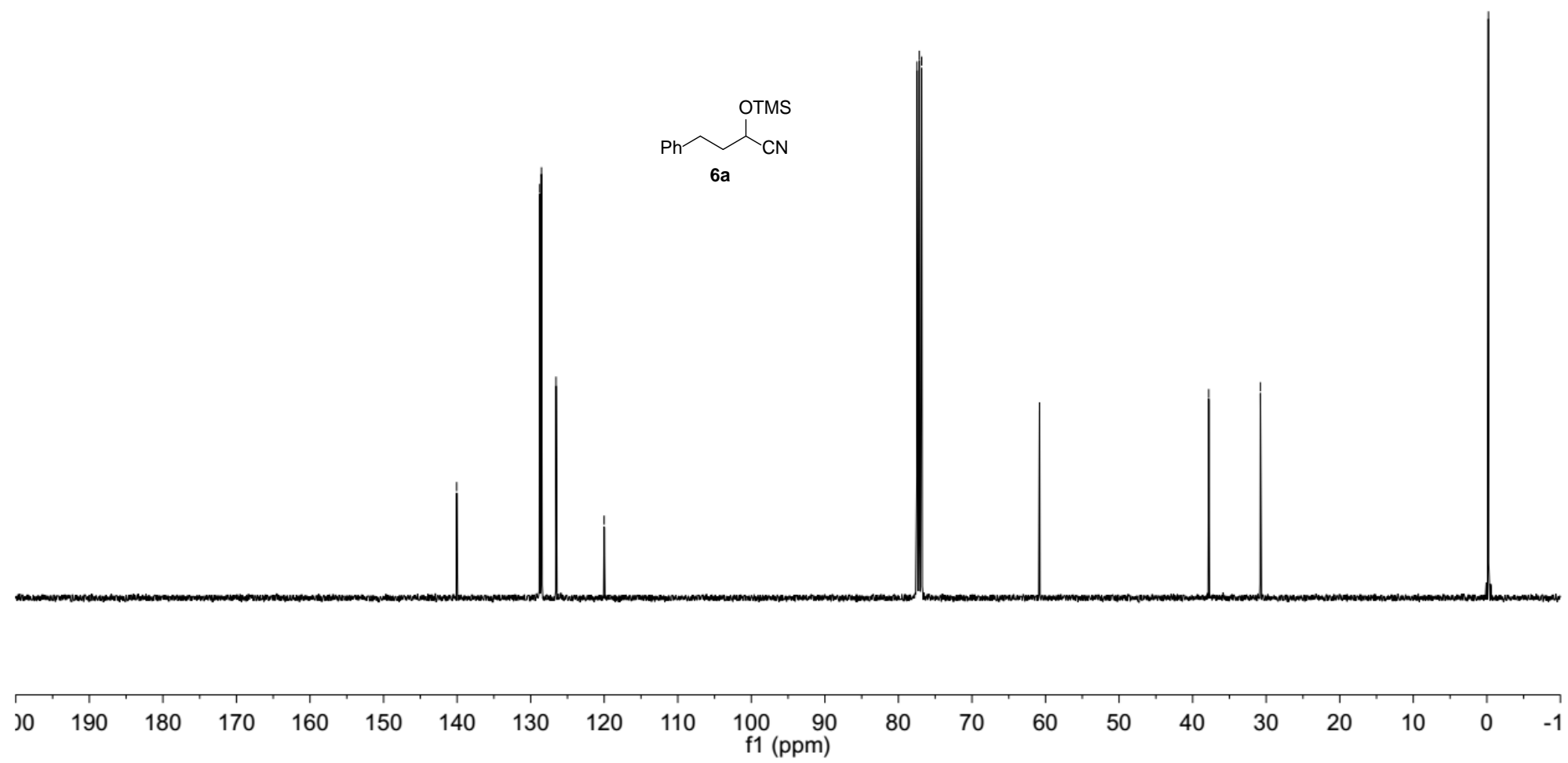

S70 


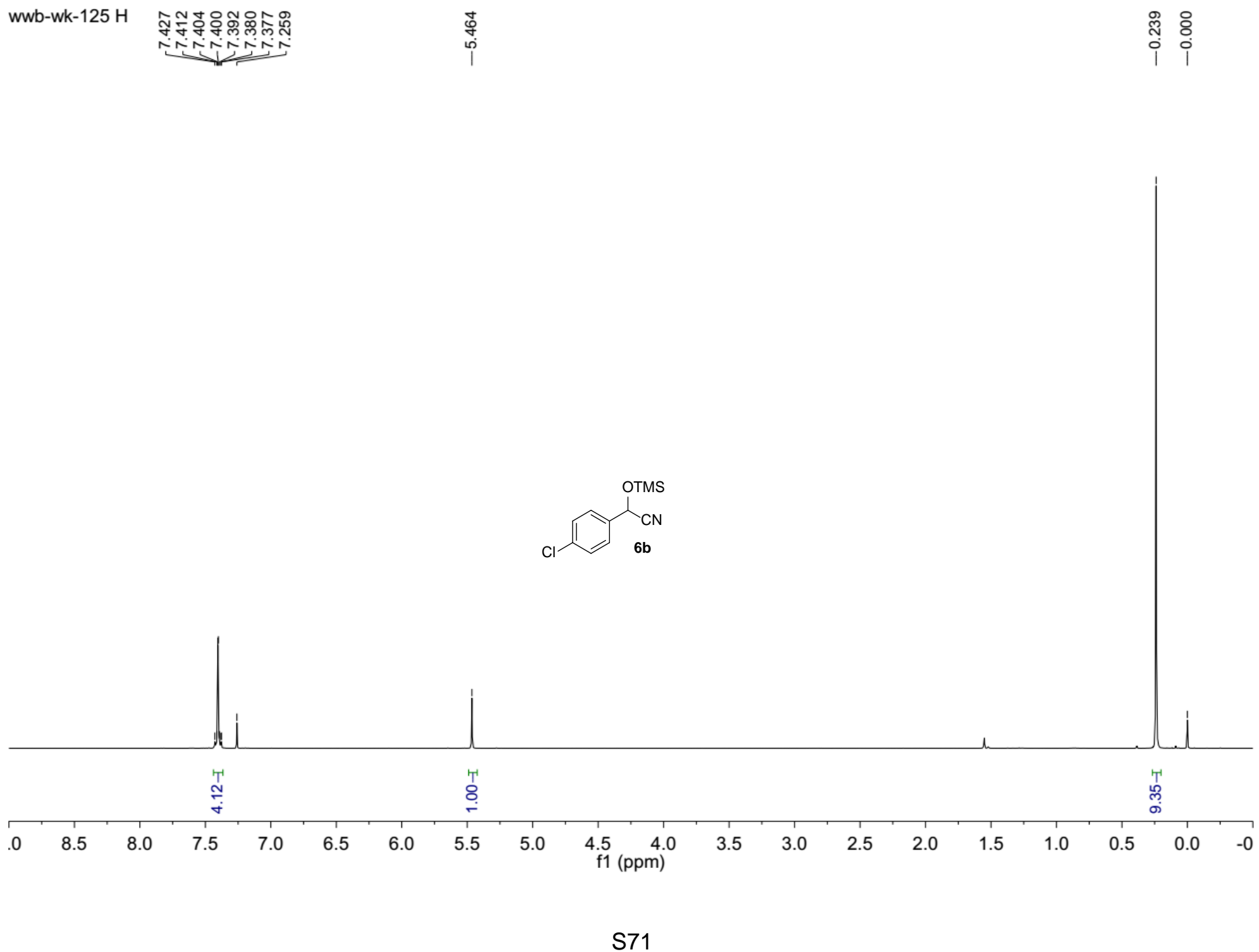




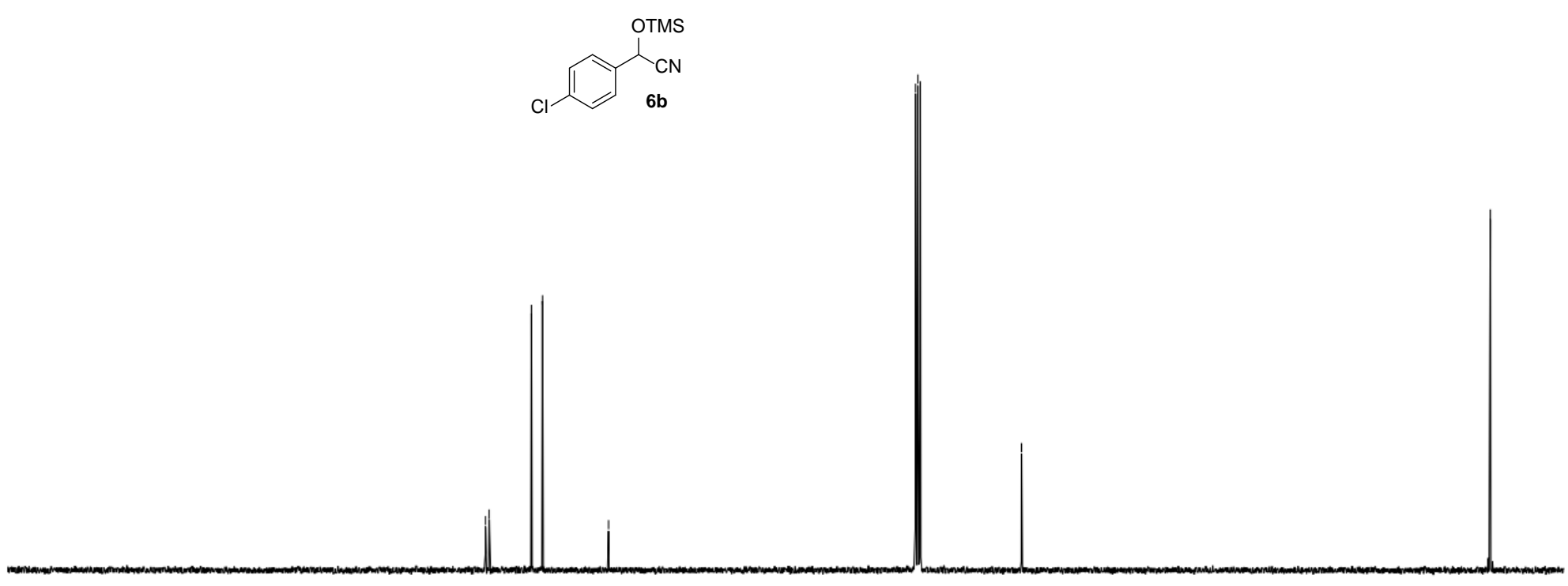

$\begin{array}{llllllllllll}0 & 190 & 180 & 170 & 160 & 150 & 140 & 130 & 120 & 110 & 100 & 90\end{array}$ f1 (ppm) 


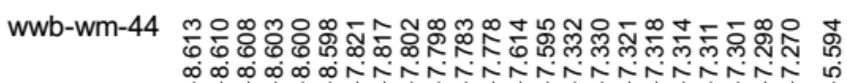

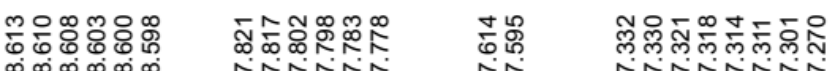

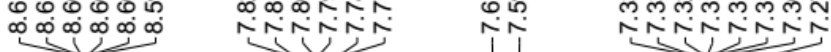

$\int_{6 c}^{\mathrm{OTMS}}$
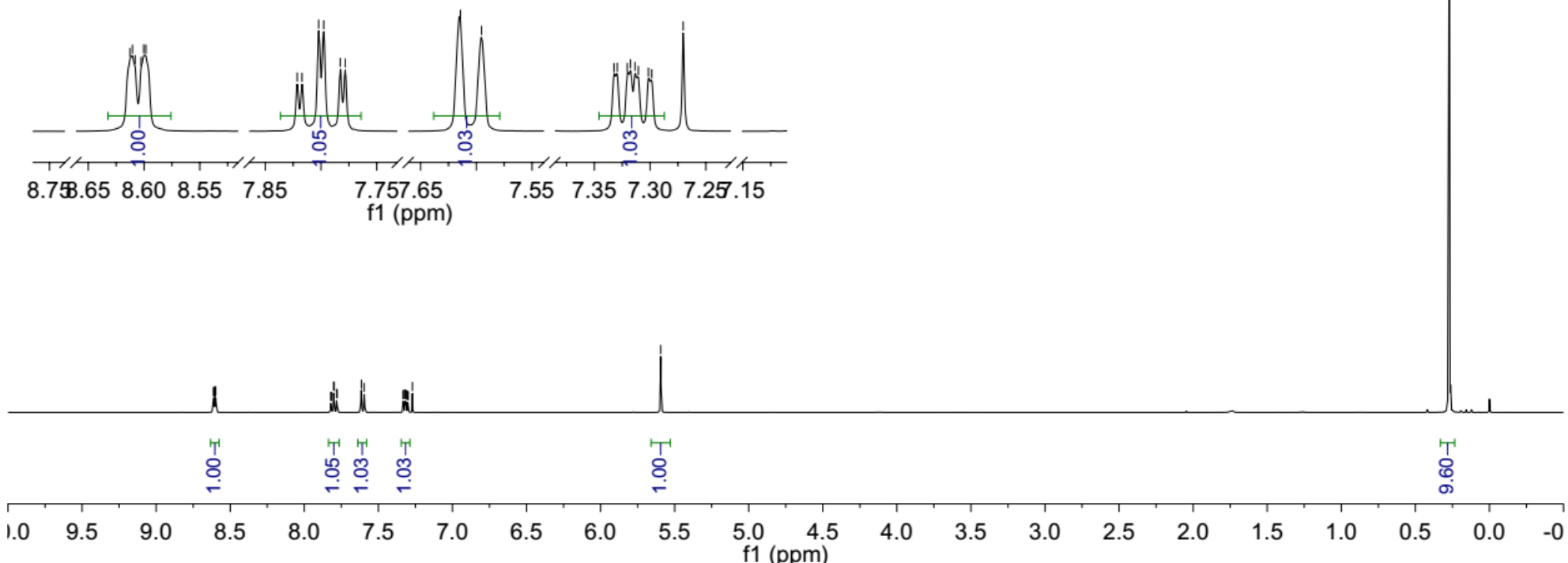
wwb-wm-44

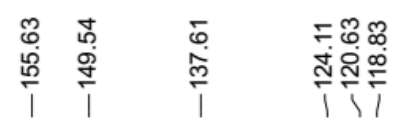

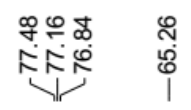

ํㅗํ
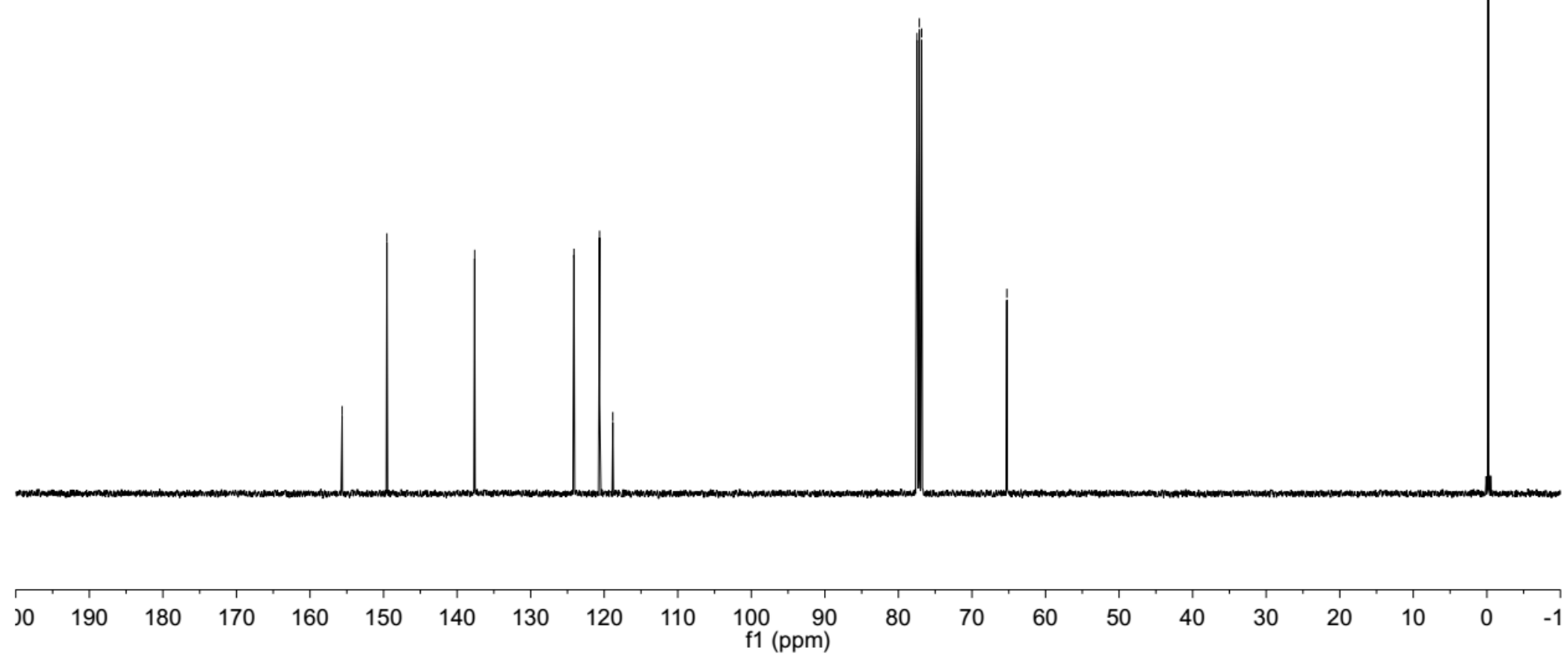

S74 


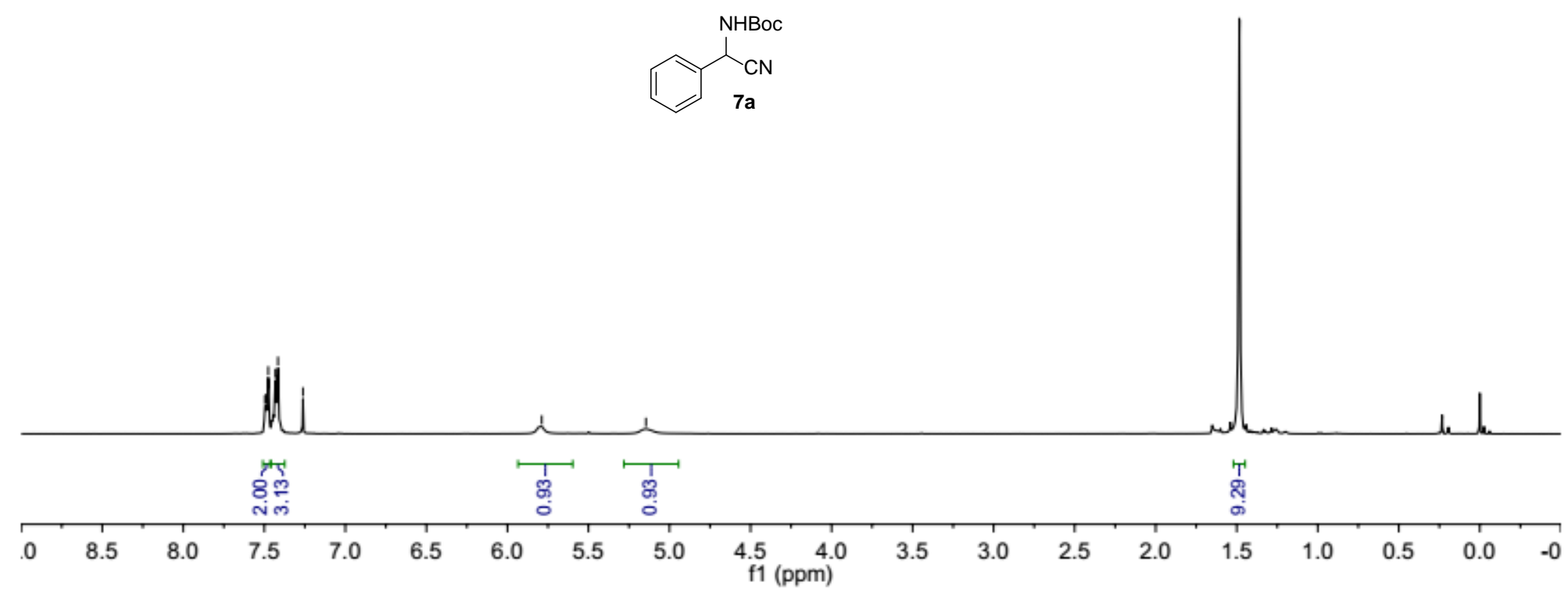




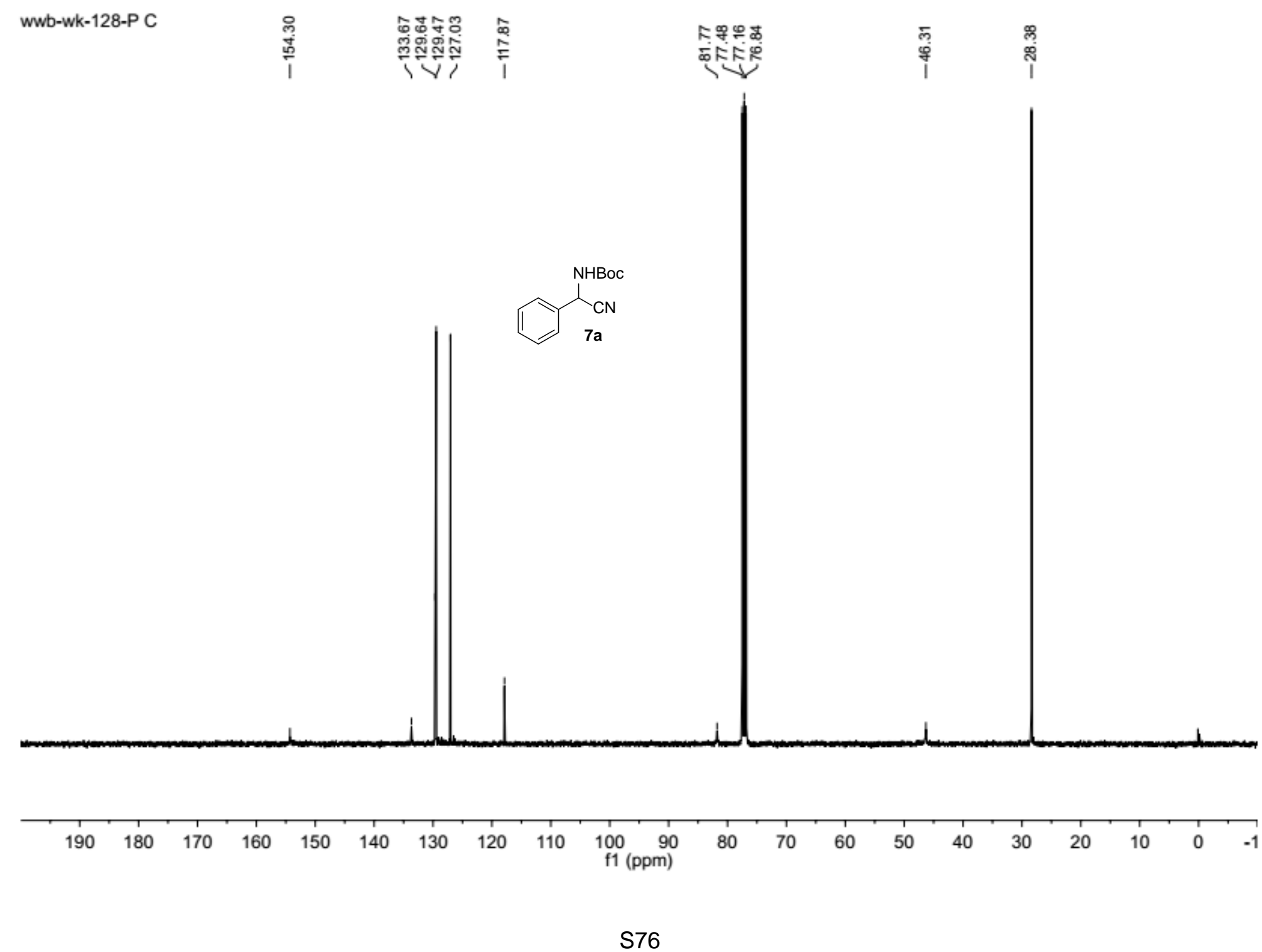


wwb-wk-118

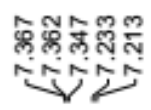

$\underset{\substack{\text { N } \\ \text { i }}}{1}$

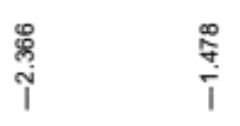

:
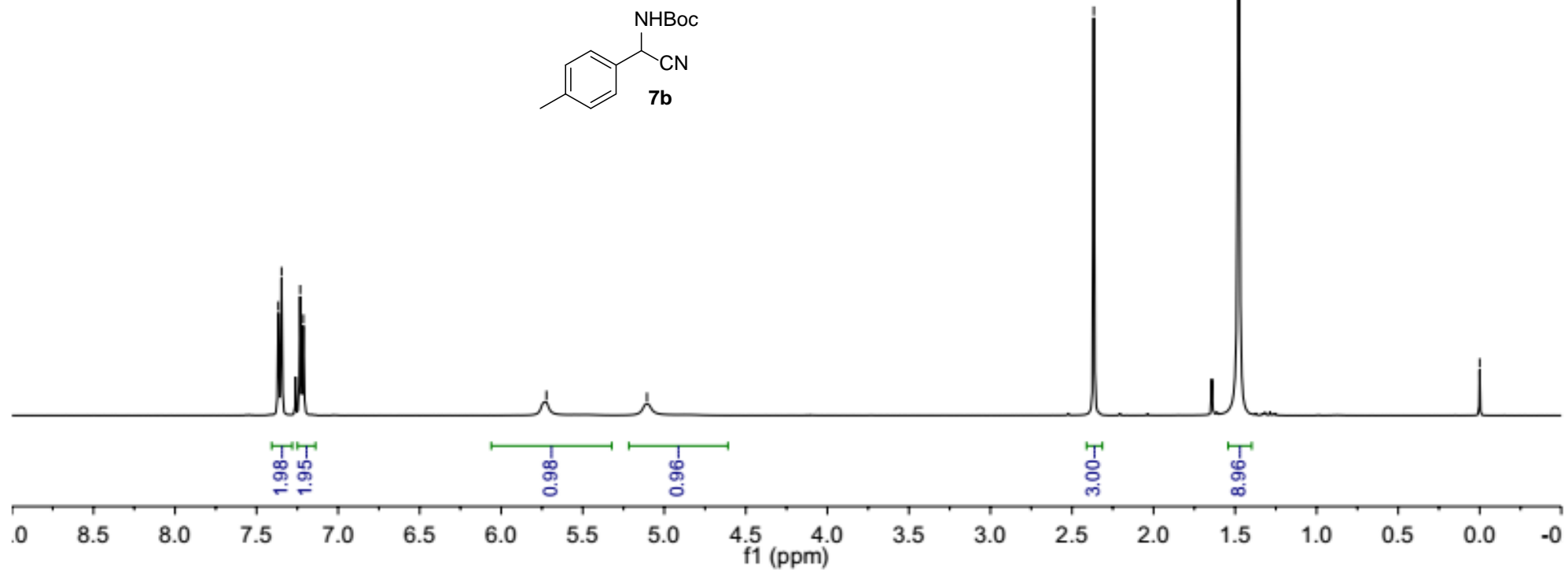

S77 

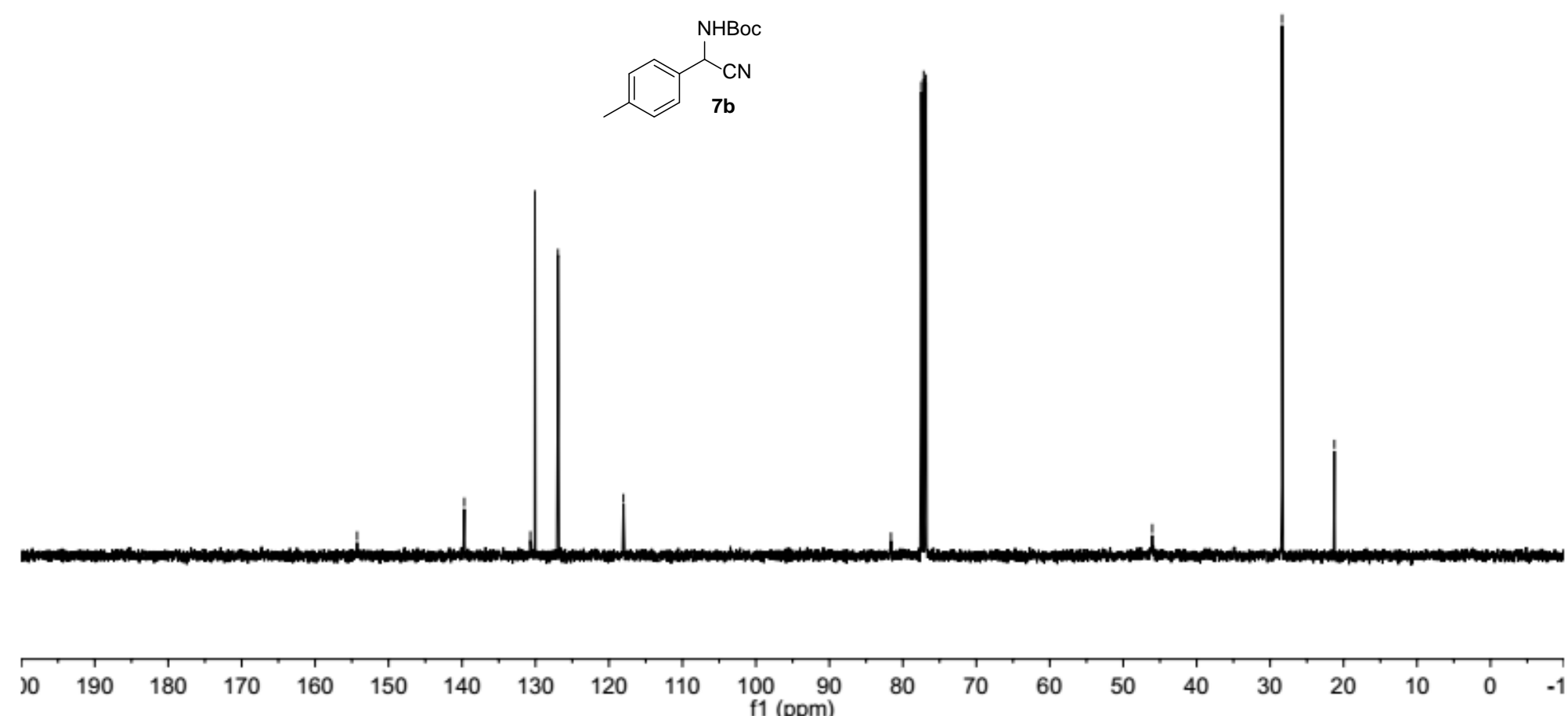
wwb-wk-126 H

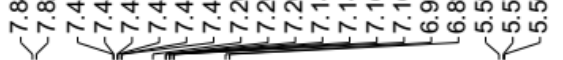

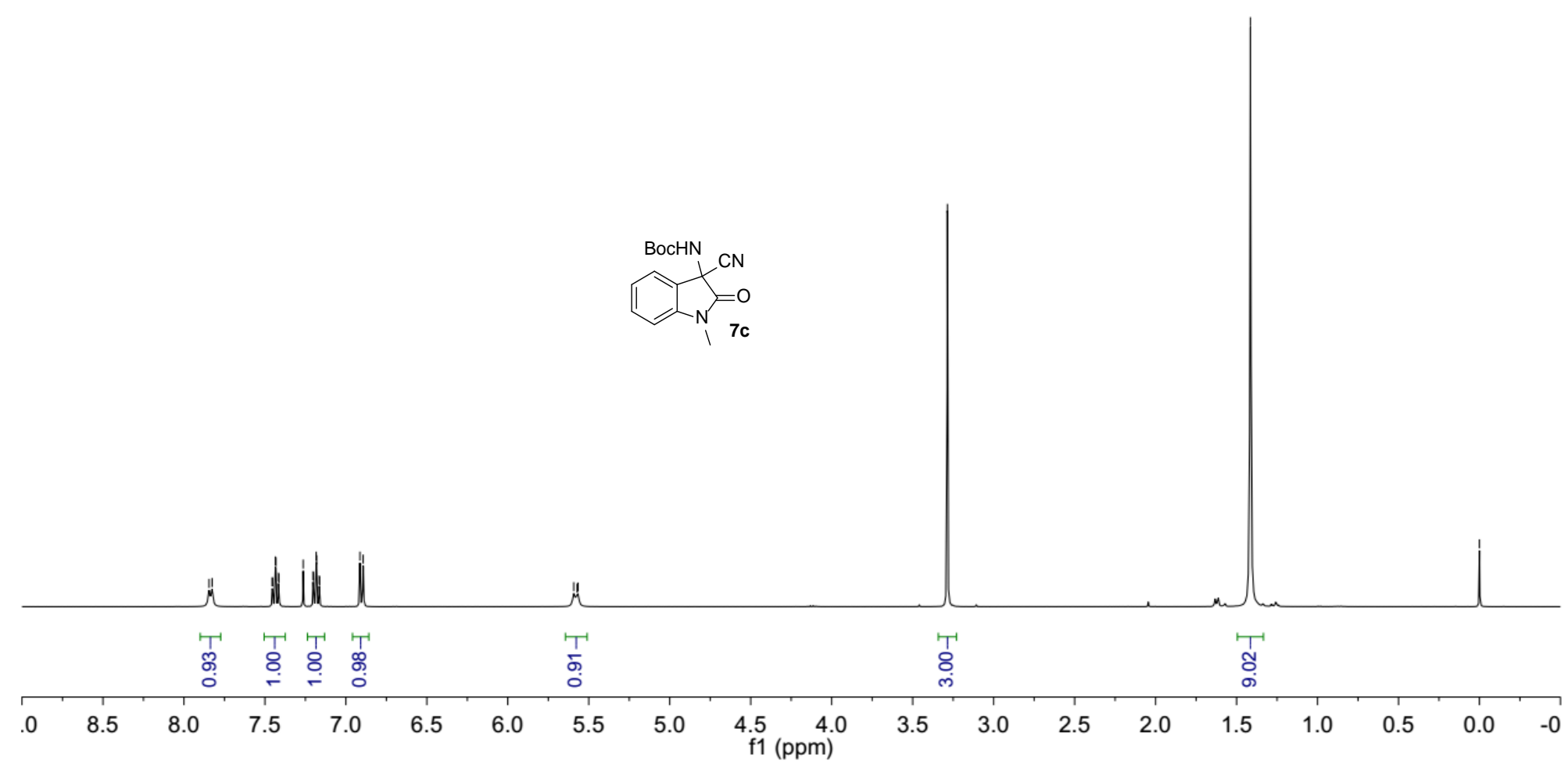




$$
\text { 而 }
$$




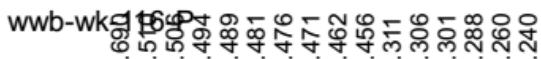

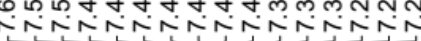

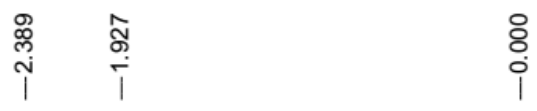

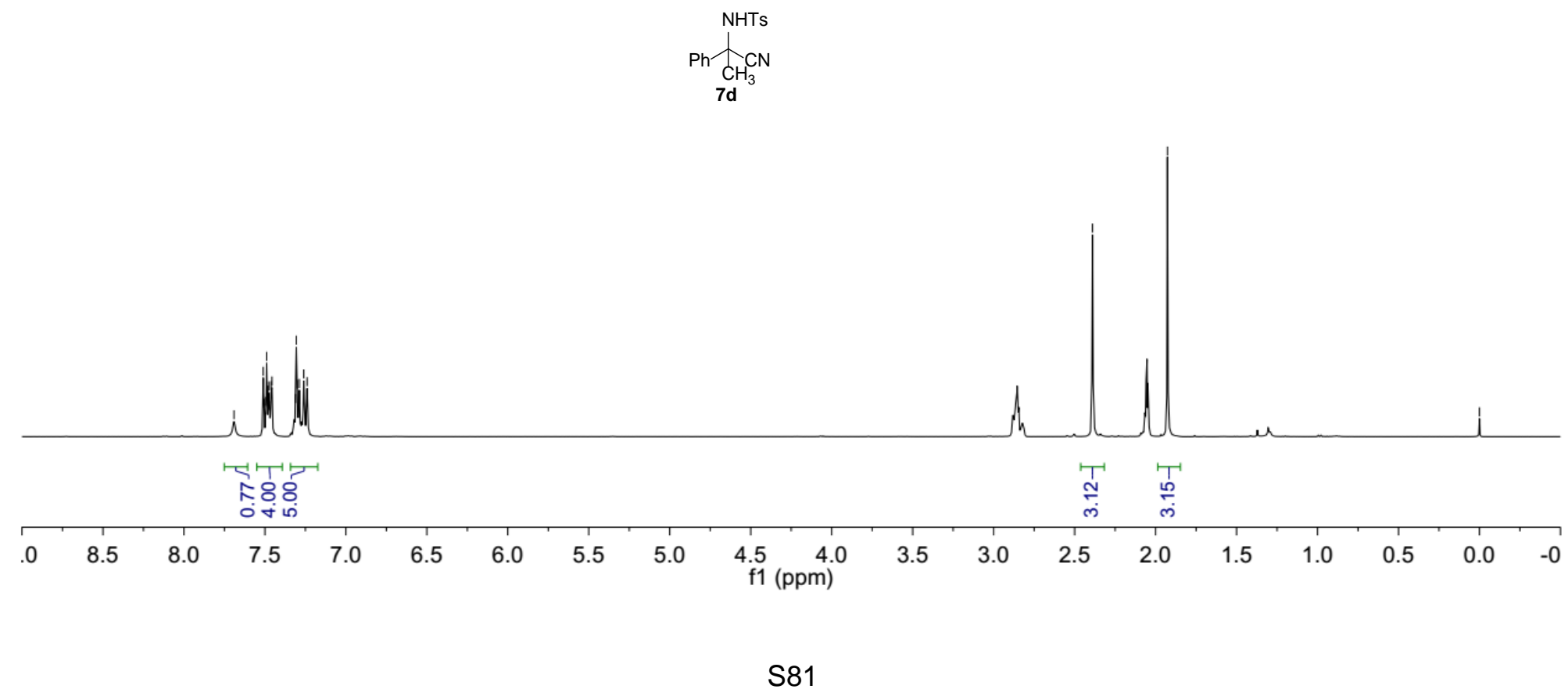


wwb-wk-\$16-P-C

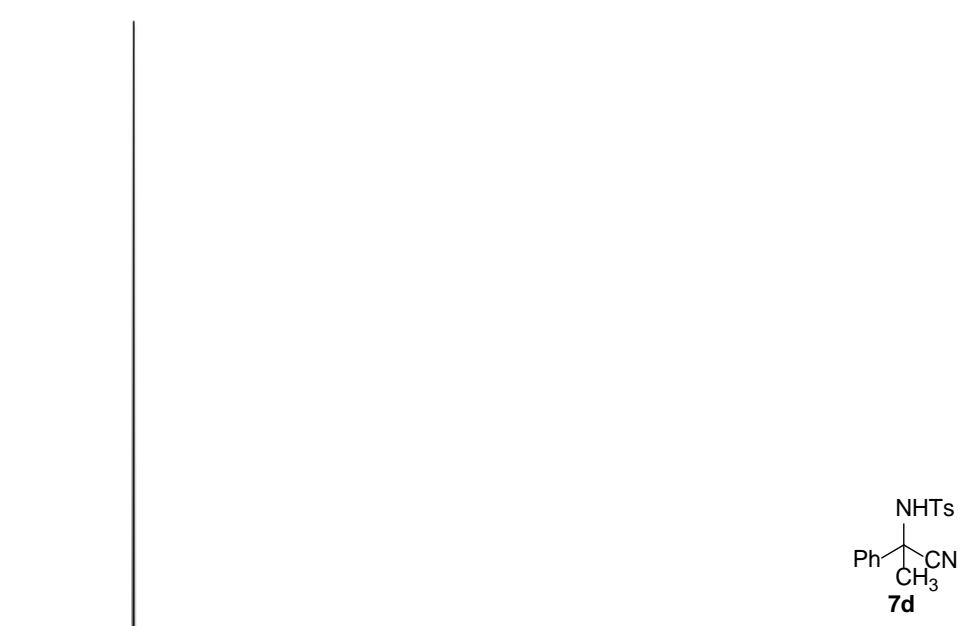

$=$ =

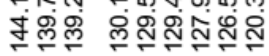

广र

$\underset{7 \mathrm{~d}}{\underset{\mathrm{CH}}{\mathrm{NHTS}}}$

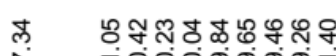

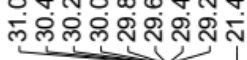

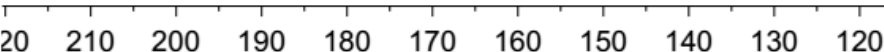

$110 \quad 100$ 